\title{
Empirical strong-line oxygen abundance calibrations from galaxies with electron-temperature measurements ${ }^{\star}$
}

\author{
S. Y. Yin ${ }^{1,3}$, Y. C. Liang ${ }^{1,2}$, F. Hammer ${ }^{2}$, J. Brinchmann ${ }^{4}$, B. Zhang ${ }^{1,3}$, L. C. Deng ${ }^{1}$, and H. Flores ${ }^{2}$ \\ 1 National Astronomical Observatories, Chinese Academy of Sciences, 20A Datun Road, Chaoyang District, Beijing 100012, \\ PR China \\ e-mail: [syyin;ycliang]@bao.ac.cn \\ 2 GEPI, Observatoire de Paris-Meudon, 92195 Meudon, France \\ 3 Department of Physicals, Hebei Normal University, Shijiazhuang 050016, PR China \\ ${ }^{4}$ CAUP, Rua das Estrelas S/N, 4150-752 Porto, Portugal
}

Received 10 June 2006 / Accepted 27 September 2006

\section{ABSTRACT}

\begin{abstract}
Aims. Our aims are to estimate the validity of empirical methods, such as $R_{23}, R_{23}-P, \log ([\mathrm{N}$ II $] / \mathrm{H} \alpha)(\mathrm{N} 2), \log [([\mathrm{O}$ III $] / \mathrm{H} \beta) /$ $([\mathrm{N} \mathrm{II}] / \mathrm{H} \alpha)](\mathrm{O} 3 \mathrm{~N} 2)$, and $\log ([\mathrm{S} \mathrm{II}] / \mathrm{H} \alpha)(\mathrm{S} 2)$, and to re-derive (or add) the calibrations of $R_{23}, \mathrm{~N} 2, \mathrm{O} 3 \mathrm{~N} 2$, and $\mathrm{S} 2$ indices for oxygen abundances on the basis of a large sample of galaxies with $T_{\mathrm{e}}$-based abundances.

Methods. We determined the gas-phase oxygen abundance for a sample of 695 galaxies and $\mathrm{H}$ II regions with reliable detections of [O III $] 4363$, using the reliable and direct temperature-sensitive $\left(T_{\mathrm{e}}\right)$ method of measuring metallicity. We selected 531 star-forming galaxies from the SDSS-DR4 database with strong emission lines, including [O III]4363 detected at a signal-to-noise ratio higher than $5 \sigma$, as well as 164 galaxies and $\mathrm{H}$ II regions from the literature with $T_{\mathrm{e}}$ measurements. The $\mathrm{O} / \mathrm{H}$ abundances were derived from a two-zone model for the temperature structure, assuming a relationship between high ionization and low ionization species.

Results. We compare our $(\mathrm{O} / \mathrm{H})_{T_{\mathrm{e}}}$ measurements of the SDSS sample with the abundances obtained by the MPA/JHU group who used multiple strong emission lines and Bayesian techniques (Tremonti et al. 2004). For roughly half of the sample the Bayesian abundances are overestimated $\sim 0.34 \mathrm{dex}$, possibly due to the treatment of nitrogen enrichment in the models they used. The $R_{23}$ and $R_{23}-P$ methods systematically overestimate the $\mathrm{O} / \mathrm{H}$ abundance by a factor of $\sim 0.20$ dex and $\sim 0.06$ dex, respectively. The $\mathrm{N} 2$ index, rather than the $\mathrm{O} 3 \mathrm{~N} 2$ index, provides more consistent $\mathrm{O} / \mathrm{H}$ abundances with the $T_{\mathrm{e}}$-method, but with some scatter. The relations of $\mathrm{N} 2, \mathrm{O} 3 \mathrm{~N} 2$, and $\mathrm{S} 2$ with $\log (\mathrm{O} / \mathrm{H})$ are consistent with the photoionization model calculations of Kewley \& Doptita (2002), but $R_{23}$ does not match well. We derive analytical calibrations for $\mathrm{O} / \mathrm{H}$ from $R_{23}, \mathrm{~N} 2, \mathrm{O} 3 \mathrm{~N} 2$, and $\mathrm{S} 2$ indices on the basis of this large sample, including the excitation parameter $P$ as an additional parameter in the $\mathrm{N} 2$ calibration. These empirical calibrations are free of the systematic problems inherent in abundance calibrations based on photoionization models.

Conclusions. We conclude that the N2, O3N2, and S2 indices are useful indicators for calibrating metallicities of galaxies with $12+$ $\log (\mathrm{O} / \mathrm{H})<8.5$ and that the $R_{23}$ index works well for the metal-poor galaxies with $12+\log (\mathrm{O} / \mathrm{H})<7.9$. For the intermediate metallicity range $(7.9<12+\log (\mathrm{O} / \mathrm{H})<8.4)$, the $R_{23}$ and $R_{23}-P$ methods are unreliable for characterizing the $\mathrm{O} / \mathrm{H}$ abundances,
\end{abstract}

Key words. galaxies: abundances - galaxies: evolution - galaxies: ISM - galaxies: spiral - galaxies: starburst galaxies: stellar content

\section{Introduction}

The chemical properties of stars and gas within a galaxy provide both a fossil record of its star formation history and information on its present evolutionary status. It is therefore desirable to extract as much accurate information as possible from observations of galaxies. In particular, it is important that different methods of extracting information provide this without systematic offsets or, at the very least, that these systematic offsets are understood well. Accurate abundance measurements for the ionized gas in galaxies require the determination of the electron temperature $\left(T_{\mathrm{e}}\right)$ in this gas, which is usually obtained from the ratio of auroral to nebular line intensities, such as [O III] $\lambda \lambda 4959,5007 /[\mathrm{O}$ III $] \lambda 4363$. This is generally known as the "direct $T_{\mathrm{e}}$-method" because the electron temperature is directly inferred from observed line ratios. It is well known that this procedure is difficult to carry out for metal-rich

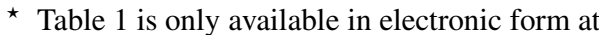
http://www . aanda.org galaxies since, as the metallicity increases, the electron temperature decreases (as the cooling is via metal lines), and the auroral lines eventually become too faint to measure. Instead, the most common method used for estimating oxygen abundance of metal-rich galaxies $(12+\log (\mathrm{O} / \mathrm{H}) \geq 8.5)$ uses the $R_{23}$ $(=([\mathrm{O}$ II $] \lambda 3727+[\mathrm{O}$ III $] \lambda \lambda 4959,5007) / \mathrm{H} \beta)$ parameter, which is the ratio of the flux in the strong optical oxygen lines to that of $\mathrm{H} \beta$ (Pagel et al. 1979; Tremonti et al. 2004 and the references therein). The $R_{23}$ indicator can also be used for metal-poor galaxies $(12+\log (\mathrm{O} / \mathrm{H})<8.5)$ (Skillman et al. 1989; Kobulnicky et al. 1999; McGaugh 1991; Pilyugin 2000; Edmunds \& Pagel 1984).

Several researchers have found, however, that $R_{23}$-derived abundances are inconsistent with the $T_{\mathrm{e}}$-derived ones, showing a systematic offset. For example, Kennicutt et al. (2003) found that $R_{23}$ overestimates the actual $\log (\mathrm{O} / \mathrm{H})$ abundance by a factor of $0.2-0.5$ dex using a sample of $20 \mathrm{H}$ II regions in $\mathrm{M} 101$ with high-S/N spectra. Some other research found similar results, for example, Bresolin et al. (2004, 2005), Garnett et al. (2004a,b), Pilyugin (2006), Shi et al. (2005, 2006). A much larger dataset 
can help to understand this effect better, as well extending it to galaxies.

To estimate abundances of galaxies, when $T_{\mathrm{e}}$ and $R_{23}$ cannot be used, some other metallicity-sensitive "strongline" ratios are very useful, for example, [N II $] \lambda 6583 / \mathrm{H} \alpha$,

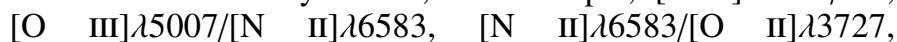
[N $\mathrm{II}] \lambda 6583 /\left[\begin{array}{ll}\mathrm{S} & \mathrm{II}\end{array}\right] \lambda \lambda 6717,6731,[\mathrm{~S}$ II] $] \lambda \lambda 6717,6731 / \mathrm{H} \alpha$, and [O III] $] \lambda 44959,5007 / \mathrm{H} \beta$ (Liang et al. 2006; Nagao et al. 2006; Pérez-Montero \& Díaz 2005; Pettini \& Pagel 2004, hereafter PP04; Denicoló et al. 2002, hereafter D02; Kewley \& Dopita 2002 , hereafter KD02). Even when $R_{23}$ is available, some of these line-ratios are useful for overcoming the well-known problem that the $R_{23}$ vs. $12+\log \mathrm{O} / \mathrm{H}$ relation is double-valued, and further information is required to break this degeneracy. Alternative methods of breaking the degeneracy when a wellestablished luminosity-metallicity relation exists or when the likelihood of each branch can be calculated, which is discussed by Lamareille et al. (2006).

Strong-line abundance indicators are typically calibrated in one of two ways: (1) using the samples of galaxies with direct ( $T_{\mathrm{e}}$-based) abundances (e.g. PP04; Pagel et al. 1979, etc.) or (2) using photoionization models (e.g. McGaugh 1991; Tremonti et al. 2004, etc.). Since the $T_{\mathrm{e}}$-method is generally thought to be the most accurate method for metallicity estimation, we will take oxygen abundances derived using this method as our baseline. We selected a large sample of 531 galaxies from the Fourth Data Release of the Sloan Digital Sky Survey database (SDSSDR4) with their [O III] $\lambda 4363$ emission line detected at an S/N higher than $5 \sigma$ and 164 associated galaxies and $\mathrm{H}$ II regions from the literature. We compared their $T_{\mathrm{e}}$-based $\mathrm{O} / \mathrm{H}$ abundances with the Bayesian estimates provided by the MPA/JHU group (shown as $\left.\log (\mathrm{O} / \mathrm{H})_{\text {Bay }}\right)$, which were obtained by fitting multi-emission lines using the photoionization models of Charlot et al. (2006), and with those derived from some strong-line ratios given in previous studies, including $R_{23}, P, \mathrm{~N} 2, \mathrm{O} 3 \mathrm{~N} 2$ methods, etc. (Kobulnicky et al. 1999; Pilyugin 2001; PP04). We also compared the observational results with the photoionization model results of KD02 for the relations of $\mathrm{O} / \mathrm{H}$ vs. $R_{23}, \mathrm{~N} 2, \mathrm{O} 3 \mathrm{~N} 2$ and $\mathrm{S} 2$ indices.

Our particular concern in this study is the abundance calibrations of strong-line ratios on the basis of their $T_{\mathrm{e}}$-based metallicities. When we compare the observational relations of $\mathrm{O} / \mathrm{H}$ vs. strong-line ratios with the photoionization model results of KD02, we find that only the N2, O3N2, S2 indices are useful for estimating abundances for galaxies with low metallicity, $12+\log (\mathrm{O} / \mathrm{H})_{T_{\mathrm{e}}}<8.5$, while other line ratios, such as $[\mathrm{N} \mathrm{II}] /[\mathrm{O}$ II $],[\mathrm{N}$ II $] /[\mathrm{S} \mathrm{II}]$, and $[\mathrm{O} \mathrm{III}] / \mathrm{H} \beta$ are not good indicators for this metallicity range due to their insensitivity to metallicities there, except in the extremely metal-poor environments (e.g. $12+\log (\mathrm{O} / \mathrm{H})<7.5$ or 7.0$)$ (Stasińska 2002; KD02). The value of $R_{23}$ is a useful indicator of metallicity for the low metallicity region with $12+\log (\mathrm{O} / \mathrm{H})<7.9$. Thus, we will only calibrate the relationships of the $R_{23}, \mathrm{~N} 2, \mathrm{O} 3 \mathrm{~N} 2$, and $\mathrm{S} 2$ indices to $\mathrm{O} / \mathrm{H}$ abundances from the observational data in this study. Moreover, for the N2 index, we follow Pilyugin $(2000,2001 \mathrm{a}, \mathrm{b})$ to add the excitation parameter $P(=[\mathrm{O} \mathrm{III}] /([\mathrm{O}$ II $]+[\mathrm{O}$ III $]))$ to separate the sample galaxies into three sub-samples in the calibrations. These calibrations can be the extension of the metal-rich region studied by Liang et al. (2006) to the low-metallicity region.

This paper is organized as follows. The sample selection criteria are described in Sect. 2. The determinations of the oxygen abundances from $T_{\mathrm{e}}$ are presented in Sect. 3. In Sect. 4, we present the comparisons between the $(\mathrm{O} / \mathrm{H})_{T_{\mathrm{e}}}$ and the $(\mathrm{O} / \mathrm{H})_{\mathrm{Bay}}$, as well as those abundances derived from other strong-line relations. Section 5 shows the comparison of the observational data with the photoionization models of KD02. In Sect. 6, we rederive analytical calibrations between $\mathrm{O} / \mathrm{H}$ and $R_{23}, \mathrm{~N} 2, \mathrm{O} 3 \mathrm{~N} 2$, $\mathrm{S} 2$ indices, as well the two-parameter calibrations for the $\mathrm{N} 2$ index with the $P$-parameter included. The conclusions are given in Sect. 7.

\section{Observational data}

We selected 531 galaxies from the SDSS-DR4 (AdelmanMcCarthy et al. 2006) and gathered 164 low-metallicity H II regions and galaxies from the literature in this study.

\subsection{The SDSS-DR4 data}

The SDSS-DR4 provides spectra in the wavelength range 3800-9200 $\AA$ for $>500000$ galaxies over 4783 square degrees ${ }^{1}$. The MPA-JHU collaboration has in addition measured emission-line fluxes and some derived physical parameters for a sample of 520082 unique galaxies at the MPA SDSS website ${ }^{2}$. Therefore, we call the working sample selected in this study as the "MPA/JHU sample" hereafter. We have selected the "starforming galaxies" with metallicity measurements and identified them following the selection criteria of the traditional line diagnostic diagram [N II] $/ \mathrm{H} \alpha$ vs. [O III] $/ \mathrm{H} \beta$ (Baldwin et al. 1981; Veilleux \& Osterbrock 1987; Kewley et al. 2001; Kauffmann et al. 2003). The fluxes of emission-lines were measured from the stellar-feature-subtracted spectra with the spectral population synthesis code of Bruzual \& Charlot (2003; Brinchmann et al. 2004; Tremonti et al. 2004).

We selected the galaxies with redshifts $0.03<z<0.25$ to make certain to cover from [O II] to $\mathrm{H} \alpha$ and [S II] emission lines. Tremonti et al. (2004) also discuss the weak effect of aperture on estimated metallicities of the sample galaxies with $0.03<z<$ 0.25 , and this was discussed further by Kewley et al. (2005), but for the present study the aperture effects are unimportant.

In this study, we used the $T_{\mathrm{e}}$-method to derive $\mathrm{O} / \mathrm{H}$ abundances of the sample galaxies (see Sect. 3 for details); therefore, we selected the samples with the [O III] $\lambda 4363$ emissionline detected where the $S / N$ is higher than $5 \sigma$. To be consistent with Liang et al. (2006) and Tremonti et al. (2004), we also considered the objects with measured fluxes of [O II] $\lambda \lambda 3726,3729$, [O III] $\lambda 5007, \mathrm{H} \beta, \mathrm{H} \alpha$, [N II] $\lambda 6583$, [S II] $\lambda \lambda 6717,6731$ emission lines, and the $S / N$ of $\mathrm{H} \beta, \mathrm{H} \alpha,[\mathrm{N}$ II], [S II] are larger than $5 \sigma$. The final sample consists of 531 galaxies. These have fluxes in [O III] $\lambda 4363$ greater than $5.3 \times 10^{-17} \mathrm{erg} \mathrm{s}^{-1} \mathrm{~cm}^{-2}$, with a mean value of $21.27 \times 10^{-17} \mathrm{erg} \mathrm{s}^{-1} \mathrm{~cm}^{-2}$.

The fluxes of the emission lines are corrected for dust extinction, which are estimated using the Balmer-line ratio $\mathrm{H} \alpha / \mathrm{H} \beta$, and assuming case $\mathrm{B}$ recombination, with a density of $100 \mathrm{~cm}^{-3}$ and a temperature of $10^{4} \mathrm{~K}$, and the intrinsic ratio of $\mathrm{H} \alpha / \mathrm{H} \beta$ is 2.86 (Osterbrock 1989), with the relation of $\left(\frac{I_{\mathrm{H} \alpha}}{I_{\mathrm{H} \beta}}\right)_{\mathrm{obs}}=$ $\left(\frac{I_{\mathrm{H} \alpha 0}}{I_{\mathrm{H} \beta 0}}\right)_{\text {intr }} 10^{-c(f(\mathrm{H} \alpha)-f(\mathrm{H} \beta))}$. Using the average interstellar extinction law given by Osterbrock(1989), we have $f(\mathrm{H} \alpha)-f(\mathrm{H} \beta)=$ -0.37 . For the 56 data points with $c<0$, we assume they have $c=0$ since their intrinsic $\mathrm{H} \alpha / \mathrm{H} \beta$ may be lower than 2.86 if their electron temperature is high (Osterbrock 1989, p. 80).

Nearly all previous empirical oxygen abundance calibrations were derived from individual $\mathrm{H}$ II regions since it is much easier

\footnotetext{
1 http://www.sdss.org/dr4/

http://www.mpa-garching.mpg.de/SDSS/
} 
to detect [O III]4363 that way. In contrast, the SDSS data samples the inner few kpc of most galaxies. One question is whether the global spectrum from a mixture of different $\mathrm{H}$ II regions will yield meaningful average abundances of the galaxies or not. Kobulnicky et al. (1999), Moustakas \& Kennicutt (2006), and Pilyugin et al. (2004) all conclude that the spatially unresolved emission-line spectra can reliably indicate the chemical properties of distant star-forming galaxies. However, as Kobulnicky et al. (1999) mention, the standard nebular chemical abundance measurement methods may be subject to small systematic errors when the observed volume includes a mixture of gas with diverse temperatures, ionization parameters, and metallicities. For the low-mass, metal-poor galaxies, such as those we are studying in this work, standard chemical analyses using global spectra will overestimate the electron temperatures $T_{\mathrm{e}}$ due to the nonuniform $T_{\mathrm{e}}$ and large variations in the ionization parameter since the global spectra are biased toward the objects with stronger emission lines. As a result, the oxygen abundances derived from $T_{\mathrm{e}}$ will be underestimated, i.e. about $<0.1 \mathrm{dex}$ in $\log (\mathrm{O} / \mathrm{H})$ (for more massive metal-rich galaxies like local spiral galaxies, there is about \pm 0.2 dex discrepancy). However, since this bias is small, and not well-constrained for our dataset, we do not attempt to correct for it.

\subsection{The metal-poor galaxies from the literature}

In addition to the MPA/JHU samples, we also collected 164 low-metallicity samples including some blue compact galaxies (BCDs) and $\mathrm{H}$ II regions from the literature, which were taken from Izotov et al. (1994, 1996, 1997a,b, 1999, 2001a,b, 2004), Izotov \& Thuan (1998a,b, 1999, 2004), van Zee (2000), Kniazev et al. (2000), Vilchez et al. (2003), Guseva et al. (2003a,b,c), Melbourne et al. (2004), and Lee et al. (2004). There are 110 $\mathrm{H}$ II regions and 54 galaxies in this sample.

We re-estimated their $\mathrm{O} / \mathrm{H}$ abundances by using the electronic temperatures method given in Sect. 3. Their metallicities are $7.1<12+\log (\mathrm{O} / \mathrm{H})<8.4$, more metal-poor than the SDSS galaxies generally. To check if there is systematic difference between our estimates and the values given in the previous studies, we compared their $T_{\mathrm{e}}$-based oxygen abundances obtained by us with those $T_{\mathrm{e}}$-based given in the original reference in Fig. 1, which shows that they are very consistent, and the very slight difference may come from different atomic data.

\section{Abundance determination from $T_{\mathrm{e}}$}

A two-zone model for the temperature structure within the H II region was adopted. In this model, $T_{\mathrm{e}}$ ([O III]) is taken to represent the temperature for high-ionization species such as $\mathrm{O}^{++}$, while $T_{\mathrm{e}}([\mathrm{O} \mathrm{II}])$ is used for low-ionization species such as $\mathrm{O}^{+}$. The general method is first to derive $t_{3}\left(=10^{-4} T_{\mathrm{e}}([\mathrm{O} \mathrm{III}])\right)$ from the emission-line ratio of [O III $] 4959,5007 /[\mathrm{O}$ III $] 4363$ and then to estimate $t_{2}\left(=10^{-4} T_{\mathrm{e}}([\mathrm{O} \mathrm{II}])\right)$ from an analytical relation between $t_{2}$ and $t_{3}$ inferred from photoionization calculations.

Izotov et al. (2006) published a set of equations for the determination of the oxygen abundances in $\mathrm{H}$ II regions for a fivelevel atom. They used the atomic data from the references listed in Stasińska (2005). According to those authors, the electron temperature $t_{3}$ (in units of $10^{4} \mathrm{~K}$ ) and the ionic abundances $\mathrm{O}^{++} / \mathrm{H}^{+}$and $\mathrm{O}^{+} / \mathrm{H}^{+}$are estimated as follows:

$$
t_{3}=\frac{1.432}{\log ((\lambda 4959+\lambda 5007) / \lambda 4363)-\log C_{T}},
$$

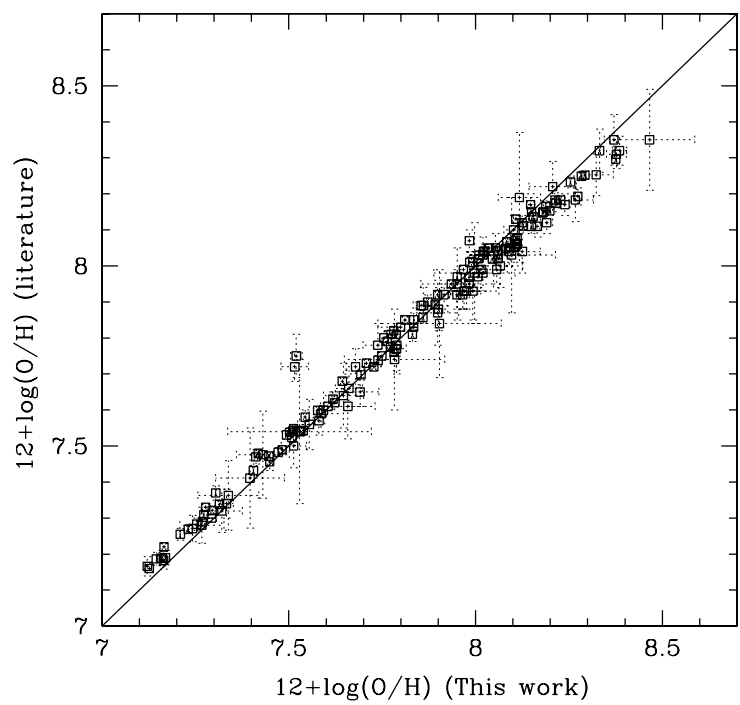

Fig. 1. Comparison between the $T_{\mathrm{e}}$-based oxygen abundances (this work) and those from the literature.

where

$C_{T}=\left(8.44-1.09 t_{3}+0.5 t_{3}^{2}-0.08 t_{3}^{3}\right) \frac{1+0.0004 x_{3}}{1+0.044 x_{3}}$,

with $x_{3}=10^{-4} n_{\mathrm{e}} t_{3}^{-1 / 2}$, and $n_{\mathrm{e}}$ is the electron density in $\mathrm{cm}^{-3}$. And

$$
\begin{array}{r}
12+\log \left(\mathrm{O}^{++} / \mathrm{H}^{+}\right)=\log \left(I_{[\mathrm{OIII}] \lambda 4959+\lambda 5007} / I_{\mathrm{H} \beta}\right) \\
+6.200+\frac{1.251}{t_{3}}-0.55 \log t_{3}-0.014 t_{3},
\end{array}
$$

$$
\begin{array}{r}
12+\log \left(\mathrm{O}^{+} / \mathrm{H}^{+}\right)=\log \left(I_{[\mathrm{OIII}] \lambda 3726+\lambda 3729} / I_{\mathrm{H} \beta}\right) \\
+5.961+\frac{1.676}{t_{2}}-0.40 \log t_{2}-0.034 t_{2} \\
+\log \left(1+1.35 x_{2}\right)
\end{array}
$$

with $x_{2}=10^{-4} n_{\mathrm{e}} t_{2}^{-1 / 2}$, and $n_{\mathrm{e}}$ is the electron density in $\mathrm{cm}^{-3}$. The total oxygen abundances are then derived from the following equation:

$\frac{\mathrm{O}}{\mathrm{H}}=\frac{\mathrm{O}^{+}}{\mathrm{H}^{+}}+\frac{\mathrm{O}^{++}}{\mathrm{H}^{+}}$.

The electron temperature $t_{2}$ (in units of $10^{4} \mathrm{~K}$ ) of the lowionization zone is usually determined from $t_{3}$ following an equation derived by fitting $\mathrm{H}$ II region models. Several versions of this relation of $t_{2}$ vs. $t_{3}$ have been proposed. We used the one of Garnett (1992), which has been widely used:

$t_{2}=0.7 t_{3}+0.3$.

The electron densities in the ionized gas of the galaxies were calculated from the line ratios $[\mathrm{S}$ II $] \lambda 6717 /[\mathrm{S}$ II $] \lambda 6731$ by using the five-level statistical equilibrium model in the task TEMDEN contained in the IRAF/STSDAS package (de Robertis et al. 1987; Shaw \& Dufour 1995), which uses the latest atomic data. A reasonable upper limit of the ratios is 1.431 (Osterbrock 1989). However, we find that 118 out of the 531 SDSS galaxies have [S II] line ratios higher than 1.431 . For 71 of the 118 galaxies, the difference between the measured ratio and 1.431 is less than the error in the line ratio, so it is very possible that most of the high [S II] $\lambda 6717 /[$ S II] 66731 ratios come from errors. Therefore, 
we adopt 1.431 for these galaxies. Indeed, this approximation would not affect the ionic abundances much. The reasons are: the term containing $x_{3}$ is never important and can be omitted in calculating $C_{T}$, since $n_{\mathrm{e}}$ is always smaller than $10^{3} \mathrm{~cm}^{-3}$, and $x_{2}$ is also insignificant since it is generally less than 0.1 with $n_{\mathrm{e}}<10^{3} \mathrm{~cm}^{-3}$.

Oxygen abundances of all the 695 samples (531 from SDSS-DR4 and 164 from the literature) were estimated using the relations outlined above. The whole sample mostly shows a metallicity range of $7.1<12+\log (\mathrm{O} / \mathrm{H})_{T_{\mathrm{e}}}<8.5$. Most of the SDSS-DR4 galaxies lie in the more metal-rich region, i.e., $7.6<$ $12+\log (\mathrm{O} / \mathrm{H})_{T_{\mathrm{e}}}<8.5$, and only 63 of them have lower metallicities than $12+\log (\mathrm{O} / \mathrm{H})_{T_{\mathrm{e}}}=8.0$. Most of the samples from the literature have lower metallicities, i.e., $12+\log (\mathrm{O} / \mathrm{H})<8.0$. The typical uncertainty of the estimates is about $0.044 \mathrm{dex}$ in $12+\log (\mathrm{O} / \mathrm{H})$ and about $500 \mathrm{~K}$ in $T_{\mathrm{e}}([\mathrm{O} \mathrm{III}])$. All the data for the SDSS galaxies are given in Table 1.

\section{Comparisons between the $T_{\mathrm{e}}$ and strong-line oxygen abundances}

We compare the $T_{\mathrm{e}}$-based $\mathrm{O} / \mathrm{H}$ abundances of the sample galaxies with the Bayesian oxygen abundances provided by the MPA/JHU group, and those derived from other "strong-line" ratios, such as $R_{23}, R_{23}-P, \mathrm{~N} 2$, and O3N2.

\subsection{Comparison with the Bayesian metallicities obtained by the MPA/JHU group}

The MPA/JHU group used photoionization model (Charlot et al. 2006) to simultaneously fit most prominent emission lines. Based on the Bayesian technique, they have calculated the likelihood distribution of the metallicity of each galaxy in the sample by comparing with a large library of models $\left(\sim 2 \times 10^{5}\right)$ corresponding to different assumptions about the effective gas parameters, and then they adopted the median of the distribution as the best estimate of the galaxy metallicity (Tremonti et al. 2004; Brinchmann et al. 2004)

Very surprisingly, Fig. 2a shows that, for almost half of the sample galaxies $(\sim 227)$, their metallicities are overestimated by a factor of about 0.34 dex on average by using the model of Charlot et al. (2006). There is no obviously monotonic trend between the increasing discrepancy and the weakening [O III]4363 line or the decreasing $[\mathrm{O} \mathrm{III}] 4363 / \mathrm{H} \beta$ ratio. What is the reason for such obvious difference?

In fact, Charlot's model was based on Charlot \& Longhetti (2001, CL01 hereafter), which is a model for consistently computing the line emission and continuum from galaxies, based on a combination of recent population synthesis and photoionization codes of CLOUDY. What they did is to use a sample of 92 local galaxies as the observed constraints to determine the best model parameters, including metallicity $Z$, etc. In their calibrations, they used the emission-line luminosities of $\mathrm{H} \alpha, \mathrm{H} \beta$, [O II] $\lambda 3727,[\mathrm{O}$ III] $\lambda 5007,[\mathrm{~N} \mathrm{II}] \lambda 6583$, and [S II] $\lambda \lambda 6717,6731$ emission lines. The main difference between this method and the other strong line methods discussed below is that all the emission lines are used simultaneously so that any clear change in the abundance ratio $X / O$ for any element $X$ from that used in the CL01 models has the potential of causing offsets. In particular, the clear offset between $(\mathrm{O} / \mathrm{H})_{\text {Bay }}$ and $(\mathrm{O} / \mathrm{H})_{T_{\mathrm{e}}}$ is possibly related to how secondary nitrogen enrichment is treated in the CL01 models.
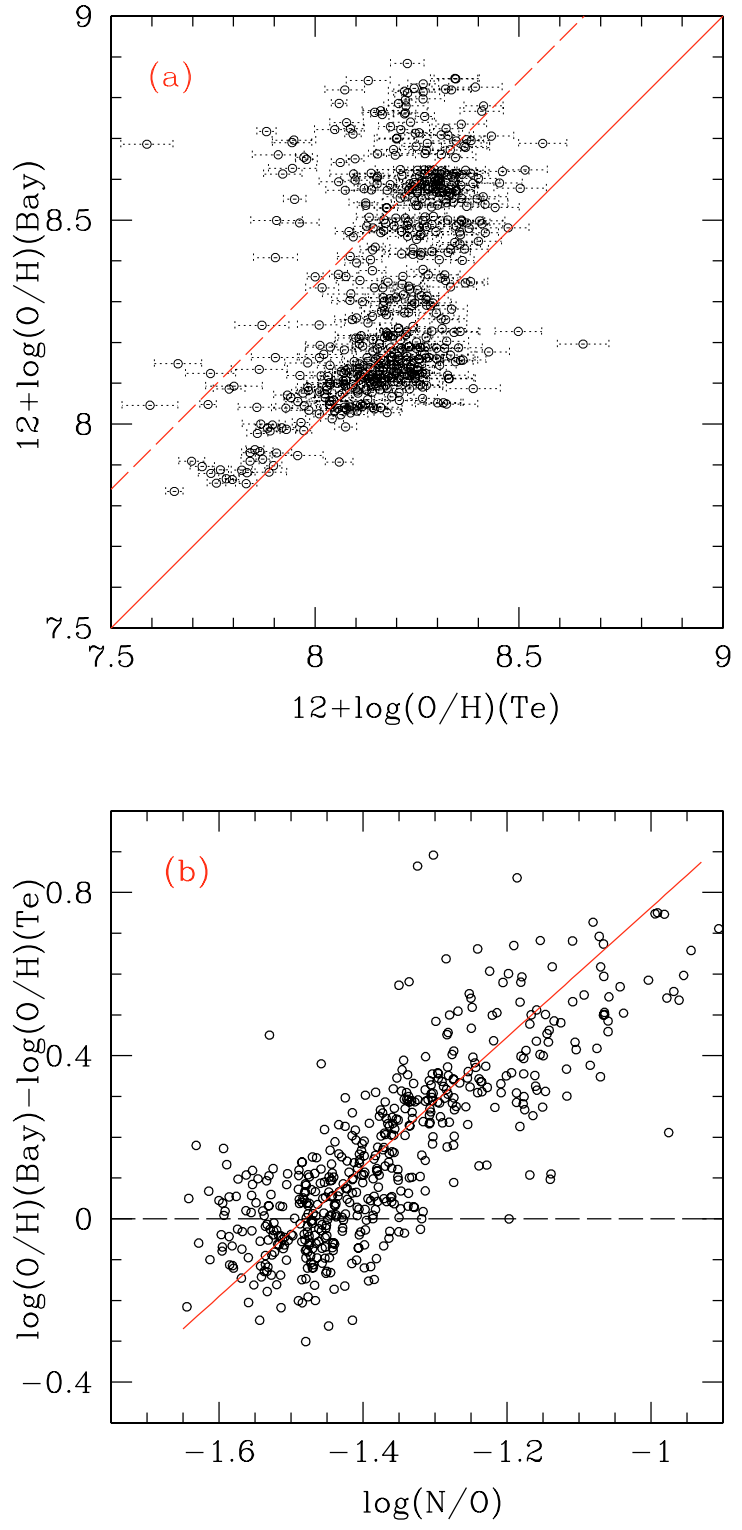

Fig. 2. a) Comparison between the electron temperature-based oxygen abundances and those Bayesian estimates obtained by the MPA/JHU group. The solid line is the equal-value line, and the long-dashed line represents a +0.34 dex discrepancy from the equal-value line to show the overestimates of the oxygen abundances by using the model of Charlot et al. (2006) for almost half of the sample galaxies. b) The offset between the $\log (\mathrm{O} / \mathrm{H})_{\text {Bay }}$ and the $\log (\mathrm{O} / \mathrm{H})_{T_{\mathrm{e}}}$ as a function of the $\log (\mathrm{N} / \mathrm{O})$ abundance ratios for the MPA/JHU sample galaxies. The solid line is the linear least-square fit for the relations, given as Eq. (7).

Figure $2 b$ shows that the "offset" between $\log (\mathrm{O} / \mathrm{H})_{\text {Bay }}$ and $\log (\mathrm{O} / \mathrm{H})_{T_{\mathrm{e}}}$ (the former minus the latter) strongly correlates with the $\log (\mathrm{N} / \mathrm{O})$ abundance ratio, which can be shown as a linear least-square fit:

offset $=1.589 \times \log (\mathrm{N} / \mathrm{O})+2.352$,

with an rms of 0.176 dex. The $\log (\mathrm{N} / \mathrm{O})$ abundance ratios are obtained from the electron temperature in the [N II] emission region (equal to the $t_{2}$ given in Sect. 3) and the flux ratio of [N II] $\lambda \lambda 6548,6584$ to [O II] $\lambda 3727$ following the method given in Thurston et al. (1996). This means that the overestimates of CL01 models for the $\mathrm{O} / \mathrm{H}$ abundances may be related to the onset of secondary $\mathrm{N}$ enrichment. There is a considerable spread in 
the onset of $\mathrm{N}$ enrichment, so that the assumption of the CL01 models of a single $\mathrm{N}$ enrichment trend is too simplistic in this transition region. The simple way to avoid this problem might be to exclude [N II] from their fitting procedure. In this transition region, the relations between the $\log (\mathrm{N} / \mathrm{O})$ and the $\log (\mathrm{O} / \mathrm{H})$ of galaxies are not monotonic, unlike the almost constant trend in the low-metallicity region or the increasing trend in the highmetallicity region (see Fig. 8 of Liang et al. 2006).

\section{2. $R_{23}$ method}

Pagel et al.(1979) first proposed the empirical abundance indicator $R_{23}$ for metal-rich galaxies. Skillman et al. (1989) went on to suggest that this indicator can also be used for metal-poor galaxies. The relationship between $\mathrm{O} / \mathrm{H}$ and $R_{23}$ has been extensively discussed in the literature (see Tremonti et al. (2004) and references therein). Different calibrations will result in slightly different oxygen abundances even for the same branch (see Fig. 3 of Tremonti et al. 2004 for comparison). A few calibrations have attempted to improve the calibration by introducing an empirical estimator of the ionization parameter as a second parameter (e.g. McGaugh 1991; Charlot \& Longhetti 2001; KD02 etc.). At present, one of the popular formulas is the calibration given by Kobulnicky et al.(1999), which includes two analytical formulas for the metal-rich and metal-poor branches, respectively, and is derived from the photoionization model of McGaugh (1991).

As mentioned in the introduction, $R_{23}$ may overestimate the actual $\mathrm{O} / \mathrm{H}$ abundances by a factor of $\sim 0.2-0.5 \mathrm{dex}$ (Kennicutt et al. 2003 etc.). On the basis of this large sample of 695 galaxies, we compare here their oxygen abundances derived from the $R_{23}$ and $T_{\mathrm{e}}$ methods. Figures $3 \mathrm{a}$ and $3 \mathrm{~b}$ show this comparison, where we use the calibration formula of Kobulnicky et al. (1999) to derive the $12+\log (\mathrm{O} / \mathrm{H})_{R_{23}}$ for the sample galaxies. In Fig. 3a for the galaxies with $12+\log (\mathrm{O} / \mathrm{H})_{T_{\mathrm{e}}}<7.95$, we use their calibration formula for metal-poor galaxies; and for the galaxies with $12+\log (\mathrm{O} / \mathrm{H})_{T_{\mathrm{e}}} \geq 8.2$, we use their formulas for the metal-rich branch. These two metallicity limits are defined following the $R_{23}-P$ method in Sect. 4.3 and some other related studies (Pilyugin 2000, 2001a,b; Shi et al. 2006). Figure 3 a shows that the $R_{23}$ method will overestimate the $\mathrm{O} / \mathrm{H}$ abundances by a factor of $\sim 0.20 \mathrm{dex}$, which is analogous to what was found by Kennicutt et al. (2003) and Shi et al. (2005, 2006). Detailed discussions of this discrepancy can be found in Kennicutt et al. (2003). For the sample galaxies with $8.0<12+\log (\mathrm{O} / \mathrm{H})_{T_{\mathrm{e}}}<8.2$, which are in the turn-over region, the calibration in Kobulnicky et al. (1999) of metal-poor branch gives $(\mathrm{O} / \mathrm{H})_{R_{23}}$ good agreement with $(\mathrm{O} / \mathrm{H})_{T_{\mathrm{e}}}$. These galaxies are omitted when we discuss the discrepancy between the $T_{\mathrm{e}^{-}}$and $R_{23}$-based metallicities, which is acceptable. Figure 3 a does not show any obvious offset between the H II regions and galaxies. Pérez-Montero \& Díaz (2005) show that the giant extragalactic $\mathrm{H}$ II regions (GEHRs) have more dispersion than the $\mathrm{H}$ II galaxies and H II regions in the Galaxy and the Magellanic clouds (their Figs. 1,3,7) since the GEHRs may have different ionization parameters and stellar effective temperatures.

To clearly illustrate the uncertainties due to the doublevalue of the $R_{23}-\mathrm{O} / \mathrm{H}$ relation, we used both of the calibration formulas for metal-poor and metal-rich branches to calculate the $12+\log (\mathrm{O} / \mathrm{H})_{R_{23}}$ abundances for the galaxies having $7.9<12+\log (\mathrm{O} / \mathrm{H})_{T_{\mathrm{e}}}<8$.4. Figure $3 \mathrm{~b}$ gives the results as the points between the two vertical dashed lines, which shows that these two different formulas will result in quite different metallicities, and the discrepancy may be up to $\sim 0.4 \mathrm{dex}$. Therefore, one should be very careful when using the $R_{23}$-method to
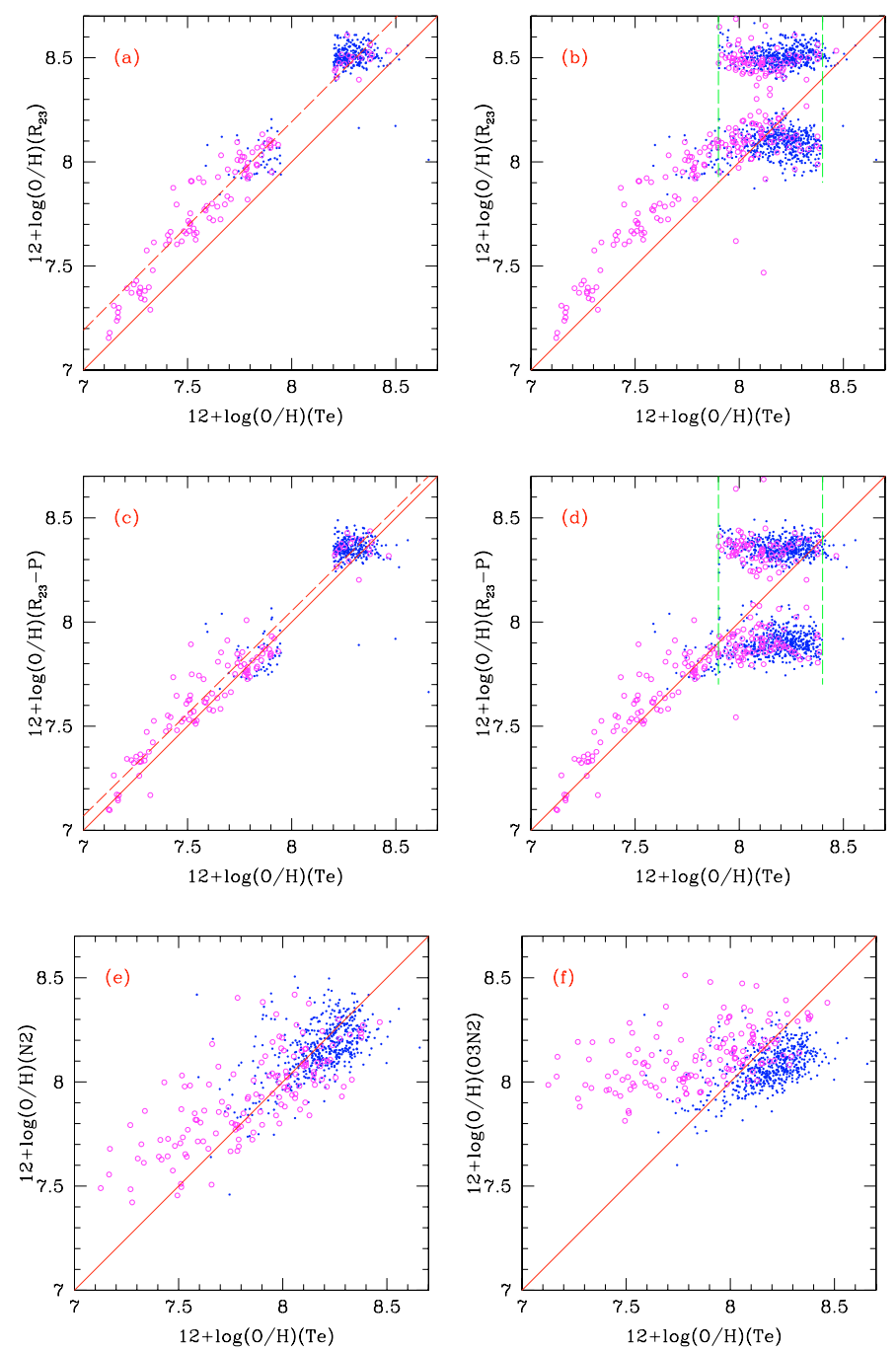

Fig. 3. Comparison between the $T_{\mathrm{e}}$-based abundances with those derived from other strong-line calibrations: a), b) $R_{23}$ method; c), d) $R_{23}-P$ method; e) N2 method; f) O3N2 method (see text). The small blue points represent the SDSS sample, and the larger magenta points (open circles) represent the sample compiled from the literature. The solid lines are the equal-value lines, and the long-dashed lines in a) and c) are the linear least-square fits to the data points, which show a discrepancy of $\sim 0.20$ dex and $\sim 0.06$ dex from the equal-value lines, respectively. The two pairs of vertical dashed lines in b) and d) show the range of $7.9<12+\log (\mathrm{O} / \mathrm{H})_{T_{\mathrm{e}}}<8.4$ for the samples whose metallicities are estimated from strong-line ratios by using two calibrations for metal-rich and metal-poor branches.

derive the metallicities of the galaxies having metallicities in the turn-over region.

\subsection{The $R_{23}-P$ method}

Pilyugin $(2000,2001 \mathrm{a}, \mathrm{b})$ suggests that the $P$ method estimates oxygen abundances of galaxies in which the oxygen abundance can be derived from two parameters, $R_{23}$ and $P$ $(=[\mathrm{O} \mathrm{III}] /([\mathrm{O} \mathrm{II}]+[\mathrm{O} \mathrm{III}]))$. Therefore, we call this method the " $R_{23}-P$ method". They derived the $\mathrm{O} / \mathrm{H}=f\left(R_{23}, P\right)$ formulas from a sample of metal-poor H II regions with $7.1<$ $12+\log (\mathrm{O} / \mathrm{H})_{T_{\mathrm{e}}}<7.95$ and a sample of moderately metal-rich $\mathrm{H}$ II regions with $8.2<12+\log (\mathrm{O} / \mathrm{H})_{T_{\mathrm{e}}}<8.7$. The best fitting relation for metal-poor objects is given as Eq. (4) in Pilyugin (2000), and the one for moderately metal-rich objects is given as Eq. (8) in Pilyugin (2001a). 
Figure $3 \mathrm{c}$ shows that there is slight difference of $\sim 0.06 \mathrm{dex}$ between the $(\mathrm{O} / \mathrm{H})_{T_{\mathrm{e}}}$ and the $(\mathrm{O} / \mathrm{H})_{R_{23}-P}$ abundances for the samples. This good agreement is indeed not surprising since Pilyugin (2000, 2001a,b) derived the $R_{23}-P$ method formulas by using a subset of the sample we have used here (Izotov et al. 1994, 1997a; Izotov \& Thuan 1998b, 1999). The minor offset is very likely caused for two reasons: (1) our larger sample than the one used by Pilyugin, which allowed us to determine the relations with higher precision; (2) the different $T_{\mathrm{e}}$-formulas and/or atomic data used in calculations. Moreover, for the low excitation samples in the low-metallicity branch with $P<0.75$, the $R_{23}-P$ method may overpredict their oxygen abundances by a factor $\sim 0.1$ dex, which is consistent with the $0.1-0.2$ dex overestimates found by van Zee \& Haynes (2006) for the lowexcitation $\mathrm{H}$ II regions in dwarf irregular galaxies.

Pilyugin \& Thuran (2005) have renewed the $R_{23}-P$ calibrations by including several improvements, such as enlarging the sample by 1 order of magnitude, etc. We used the new calibrations to re-calculate the $(\mathrm{O} / \mathrm{H})_{R_{23}-P}$ abundances for our sample galaxies and found the overstimate factor by the $R_{23}-P$ method decreases to $\sim 0.025$ dex.

Because there are no observational samples with $7.95<$ $12+\log (\mathrm{O} / \mathrm{H})<8.2$ in Pilyugin's calibration for the $R_{23}-P$ method, this method cannot provide information for the galaxies within this oxygen abundance range. To understand more about this, we calculated oxygen abundances for galaxies with $12+\log (\mathrm{O} / \mathrm{H})_{T_{\mathrm{e}}}=7.9-8.4$ using the two formulas for the metal-rich and metal-poor branches. The results are indicated in Fig. 3d. Similar to what we saw for $R_{23}$, the two branch calibrations give quite different metallicities with a difference of up to $\sim 0.4$ dex. Thus one must be very careful when applying the $R_{23}-P$ method to samples of galaxies with metallicities in the turn-over region.

\subsection{N2 method}

The $[\mathrm{N}$ II] $/ \mathrm{H} \alpha$ emission-line ratio can also be used to estimate the metallicity of galaxies. Nitrogen is mainly synthesized in intermediate- and low-mass stars. The $[\mathrm{N} \mathrm{II]} / \mathrm{H} \alpha$ ratio will therefore become stronger with ongoing star formation and galaxy evolution, until very high metallicities are reached, i.e. $12+\log (\mathrm{O} / \mathrm{H})>9.0$, where $[\mathrm{N}$ II] starts to become weaker due to the very low electron temperature caused from strong cooling by metal ions. It is worth pointing out that this ratio gives an indirect measurement of the oxygen abundance, however, since oxygen lines are not used in the method.

Following the earlier work by Storchi-Bergmann et al. (1994), and Raimann et al. (2000), D02 and PP04 suggested calibration relations for $\mathrm{O} / \mathrm{H}$ vs. $\mathrm{N} 2$ index from 236 and 137 galaxies respectively. D02 combined a sample of 128 metalrich galaxies and 108 metal-poor galaxies with [O III]4363 detected to derive their calibration. The way they estimated the metallicities of their galaxy sample was to use the $T_{\mathrm{e}^{-}}$ method for the metal-poor galaxies and the $R_{23}$ or $S_{23}$ $(=\log (([\mathrm{S}$ II $] 6717,6731+[\mathrm{S} \mathrm{III}] 9096,9531)) / \mathrm{H} \beta)$ method for the metal-rich ones. PP04 extracted the metal-poor objects from the work of D02 and updated the abundances for some of them with recent measurements by Kennicutt et al. (2003) for H II regions in M101 and by Skillman et al. (2003) for dwarf irregular galaxies in the Sculptor Group. To be consistent with the $T_{\mathrm{e}}$-method, here we use the calibration formula given by PP04 to derive the metallicities $12+\log (\mathrm{O} / \mathrm{H})_{N 2}$ for our 695 sample galaxies. Figure $3 \mathrm{e}$ gives the comparison between the $12+\log (\mathrm{O} / \mathrm{H})_{T_{\mathrm{e}}}$ and $12+\log (\mathrm{O} / \mathrm{H})_{N 2}$, which shows that the two estimates are consistent but have some scatter. The scatter may mostly come from the different ionization parameters of the galaxies and the different samples (see Sect. 6.5).

One of the advantages of using the N2 index for estimating metallicity is that the $\mathrm{N} 2$ indicator can break the degeneracy when the $R_{23}-(\mathrm{O} / \mathrm{H})$ and $\left(R_{23}, P\right)-(\mathrm{O} / \mathrm{H})$ relations are double-valued. Also, the N2 index is not affected by dust extinction since $\mathrm{H} \alpha$ and [N II] are so closely spaced in wavelength. Furthermore, near-infrared spectrographs can measure these two lines for galaxies out to high redshifts, where it is equivalent to early epochs in the evolution of galaxies (see more discussion in Liang et al. 2006).

\subsection{O3N2 method}

Another indicator of the metallicity of galaxies is $\mathrm{O} 3 \mathrm{~N} 2$ (= $\log \{([\mathrm{O}$ III $] \lambda 5007 / \mathrm{H} \beta) /([\mathrm{N}$ II $] \lambda 6583 / \mathrm{H} \alpha)\})$. It was introduced by Alloin et al.(1979) and then studied by PP04, who present one such calibration by doing a linear least-square fit for a sample of 65 (from the 137) galaxies with $-1<\mathrm{O} 3 \mathrm{~N} 2<1.9$, and provide a calibration formula. This calibration does not work for the cases of $\mathrm{O} 3 \mathrm{~N} 2>1.9$ since the data points are so scattered there in their study. Figure $3 \mathrm{f}$ shows the comparison between the $12+\log (\mathrm{O} / \mathrm{H})_{T_{\mathrm{e}}}$ and the oxygen abundances derived with the O3N2 method using the calibration of PP04 for our 695 sample galaxies. But we extrapolated to use the PP04's calibration to the galaxies with $\mathrm{O} 3 \mathrm{~N} 2>1.9$.

It is clear that this calibration will overestimate the $\mathrm{O} / \mathrm{H}$ abundances by a big factor, up to $1.0 \mathrm{dex}$, for the metal-poor galaxies with $\mathrm{O} 3 \mathrm{~N} 2>1.9$ (about $12+\log (\mathrm{O} / \mathrm{H})<8.0$ ). The scatter of the data with $-1<\mathrm{O} 3 \mathrm{~N} 2<1.9$ is 4also large, which may be due to the different ionization parameters of the galaxies.

\section{Comparisons with photoionization models}

KD02 calculated the metallicity calibrations for strong-line ratios on the basis of photoionization models. They used a combination of stellar-population synthesis and photoionization models to develop a set of ionization parameters and abundance diagnostics based on emission-line ratios from strong optical lines, with seven ionization parameters $q=5 \times 10^{6}, 1 \times 10^{7}$, $2 \times 10^{7}, 4 \times 10^{7}, 8 \times 10^{7}, 1.5 \times 10^{8}$, and $3 \times 10^{8} \mathrm{~cm} \mathrm{~s}^{-1}$. The ionization parameter $q$ is defined on the inner boundary of the nebula and can be physically interpreted as the maximum velocity of an ionization front that can be driven by the local radiation field. It will be interesting to compare the observational samples with these model results. Figure 4 shows the comparisons for the relations of $12+\log (\mathrm{O} / \mathrm{H})$ versus $R_{23}, \mathrm{~N} 2, \mathrm{O} 3 \mathrm{~N} 2$, and $\mathrm{S} 2$ indices. These relations are for the $T_{\mathrm{e}}$-based oxygen abundances of the metal-poor galaxies. Liang et al. (2006) shows such relations for the metal-rich SDSS galaxies based on $R_{23}$ abundances, and derived the corresponding calibrations (see Figs. $4 \mathrm{~b}, \mathrm{c}$ ).

Figure $4 \mathrm{a}$ shows that the significant discrepancy in the theoretically expected $R_{23}$ sequence with respect to the observed trend beginning from $12+\log (\mathrm{O} / \mathrm{H})_{T_{\mathrm{e}}}=8.0$ to lower metallicities. Nagao et al. (2006) also point this out. The observed relation of $R_{23}-\log (\mathrm{O} / \mathrm{H})$ for the data points does not show much scatter in contrast to the models of the lower branch of metallicity, which may mean a weak dependence of $R_{23}$ on ionization parameters there. In particular, the distribution of observational data is nearly vertical in an oxygen abundance range of $7.9 \sim 8.5$. The physical reason for the insensitivity of $R_{23}$ to metallicity in this metallicity region is that the $[\mathrm{O} \mathrm{III}] / \mathrm{H} \beta$ ratio (also $[\mathrm{O} \mathrm{II}] / \mathrm{H} \beta$ ) is 

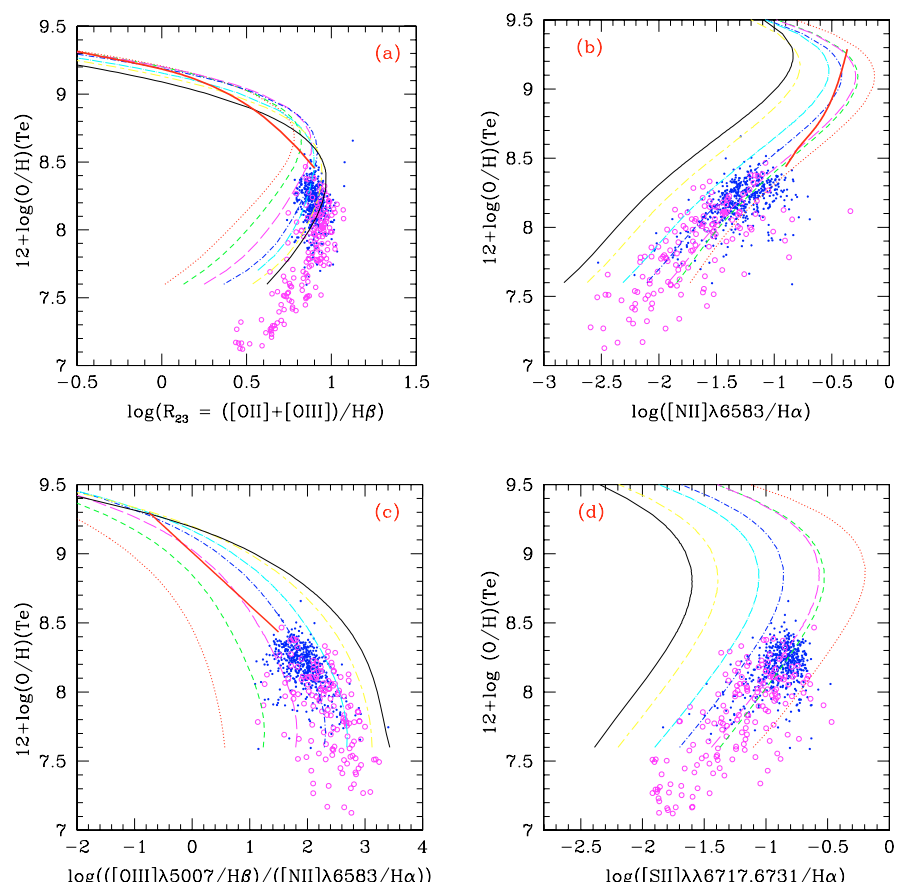

Fig. 4. Comparison between the observational data and the photoionization models of KD02. The symbols are the same as in Fig. 3. The seven lines represent the model results from KD02 with seven photoionization parameters from $q=5 \times 10^{6} \mathrm{~cm} \mathrm{~s}^{-1}$ to $q=3 \times 10^{8} \mathrm{~cm} \mathrm{~s}^{-1}$, which increase in an orderly fashion from the red dotted line to the black solid line. The solid thick line (in red) in a) is the calibration derived by Tremonti et al. (2004) for the metal-rich galaxies, and those in b) and c) are the calibrations derived by Liang et al. (2006) for the metal-rich SDSS galaxies.

independent of $T_{\mathrm{e}}$, there hence of metallicity (Stasinska 2002). It proves again that the oxygen abundance cannot be accurately measured by using $R_{23}$ in the metallicity turnover region, but the $R_{23}$ indicator works well for the metal-poor galaxies with $12+$ $\log (\mathrm{O} / \mathrm{H})<7.9$.

Figure $4 \mathrm{~b}$ shows the comparison between $12+\log (\mathrm{O} / \mathrm{H})$ and the N2 index. It shows that the observational data follow the general trend of the model results closely; namely, the $\mathrm{N} 2$ indices of galaxies increase following the increasing metallicities, up to $12+\log (\mathrm{O} / \mathrm{H})=9.0$ (by models and Liang et al. 2006). The observational data show some scatter, which may be due to their different ionization parameters. We also plot the calibration relation derived by Liang et al. (2006) with the metal-rich SDSS galaxies $(8.5<12+\log (\mathrm{O} / \mathrm{H})<9.3)$ in Fig. 4b. If we derive a calibration from the $T_{\mathrm{e}}$-based oxygen abundances of the metalpoor galaxies and extrapolate it to the metal-rich region, it will result in relatively lower $\mathrm{O} / \mathrm{H}$ abundance than when directly using the calibration from metal-rich galaxies. Perhaps one reason for this discrepancy stems from the different methods of determining metallicities of the metal-poor and metal-rich galaxies, i.e. the $T_{\mathrm{e}}$-method versus the $R_{23}$-method, or photoionization models. However, Stasińska (2005) points out that, at high metallicity, the derived $\mathrm{O} / \mathrm{H}$ values from $T_{\mathrm{e}}$ for model $\mathrm{H}$ II regions deviate strongly from the true ones; i.e., important deviations appear around $12+\log (\mathrm{O} / \mathrm{H})=8.6$ and may become huge as the metallicity increases (see their Fig. 1a).

Figure $4 \mathrm{c}$ shows the comparison between $12+\log (\mathrm{O} / \mathrm{H})$ and the $\mathrm{O} 3 \mathrm{~N} 2$ index. The general trend of the observations and of the models is similar and shows increasing $\mathrm{O} / \mathrm{H}$ abundance with decreasing $\mathrm{O} 3 \mathrm{~N} 2$ index. However, the large scatter of the data points in this plot may be due to the strong effect of the ionization parameter, and this effect is stronger in the low-metallicity region $(12+\log (\mathrm{O} / \mathrm{H})<8.5)$ than in the high-metallicity region $(12+\log (\mathrm{O} / \mathrm{H})>8.5)$. Moreover, O3N2 is much less dependent on metallicity in the low-metallicity region than in the high-metallicity region; in particular, it is almost independent of metallicity for the sample galaxies with metallicities of $12+\log (\mathrm{O} / \mathrm{H})=7.1-7.7$. This is possibly the main reason that PP04 had to limit their calibration to O3N2 < 1.9. We also present the calibration for metal-rich SDSS galaxies obtained by Liang et al. (2006) in Fig. 4c in the $8.5<12+\log (\mathrm{O} / \mathrm{H})<9.3$ region. Both the calibrations for the metal-rich and metal-poor sample galaxies are consistent with the general trend of the model predictions, but the calibration for metal-poor galaxies corresponds to relatively higher ionization parameter than the metal-rich galaxies.

Figure $4 \mathrm{~d}$ shows the comparison between $12+\log (\mathrm{O} / \mathrm{H})$ and the $\mathrm{S} 2$ index. Both the observations and models clearly show increasing $\mathrm{S} 2$ with increasing $\mathrm{O} / \mathrm{H}$. This increasing trend will extend up to $12+\log (\mathrm{O} / \mathrm{H}) \sim 8.9-9.0$, but $\mathrm{s} 2$ is affected more by the ionization parameters than the N2 index is. The large scatter of this relation may be caused by the different ionization parameters. Most of the SDSS galaxies cluster around $-1.0<$ $S 2<-0.5$. Liang et al. (2006) did not derive a metallicity calibration for S2 for the metal-rich galaxies since the data points show obvious turnover and double-valued distribution around $12+\log (\mathrm{O} / \mathrm{H}) \sim 8.9-9.0$.

In summary, Figs. $4 \mathrm{a}-\mathrm{d}$ show that $R_{23}$ is not a good metallicity indicator for the galaxies with $7.9<12+\log (\mathrm{O} / \mathrm{H})<8.5$ due to its independence of metallicity there, and it is a good indicator of the lower metallicity region with $12+\log (\mathrm{O} / \mathrm{H})<7.9 ; \mathrm{N} 2$ is a useful indicator for estimating metallicities of galaxies because it is strongly monotonicly correlated with metallicity (up to about $12+\log (\mathrm{O} / \mathrm{H}) \sim 9.0$ ); although $\mathrm{O} 3 \mathrm{~N} 2$ and $\mathrm{S} 2$ can be used to estimate metallicities of galaxies, they are more sensitive to ionization parameters than $\mathrm{N} 2$ is, and $\mathrm{O} 3 \mathrm{~N} 2$ is less dependent on metallicity than the other two. In addition, when we have the indicator of $\mathrm{O} 3 \mathrm{~N} 2$ and/or S2, normally we also have N2. Furthermore, if we can find a way to take the ionization parameters of the sample galaxies into account in the calibrations, it will provide more reliable metallicities (see Sects. 6.5, 6.6).

\section{Deriving oxygen abundance calibrations}

We obtain the $T_{\mathrm{e}}$-based $\mathrm{O} / \mathrm{H}$ abundances for the 695 sample galaxies and $\mathrm{H}$ II region. They mostly have metallicities of $7.1<12+\log (\mathrm{O} / \mathrm{H})_{T_{\mathrm{e}}}<8.5$. Then, in this section, we use their $(\mathrm{O} / \mathrm{H})_{T_{\mathrm{e}}}$ and line ratios to re-derive the oxygen abundance calibrations for the $\mathrm{N} 2, \mathrm{O} 3 \mathrm{~N} 2$ indices, and add an $\mathrm{S} 2$ index. We also derive the calibration for $R_{23}$ for the $12+\log (\mathrm{O} / \mathrm{H})_{T_{\mathrm{e}}}<$ 7.9 region. Moreover, we try to add an excitation parameter $P$ $(=[\mathrm{O} \mathrm{III}] /([\mathrm{O} \mathrm{II}]+[\mathrm{O} \mathrm{III}]))$ in the calibrations to separate the sample galaxies to be three sub-samples, which improves the N2 calibration, but does not improve the $\mathrm{O} 3 \mathrm{~N} 2$ and $\mathrm{S} 2$ calibrations much.

\subsection{The $R_{23}$ index}

The $R_{23}$ index is useful for calibrating metallicities of low metallicity galaxies with $12+\log (\mathrm{O} / \mathrm{H})<7.9$ due to its obvious correlation with $\log (\mathrm{O} / \mathrm{H})$ and weak dependence on ionization parameters there. Figure 5a shows this correlation. The linear 

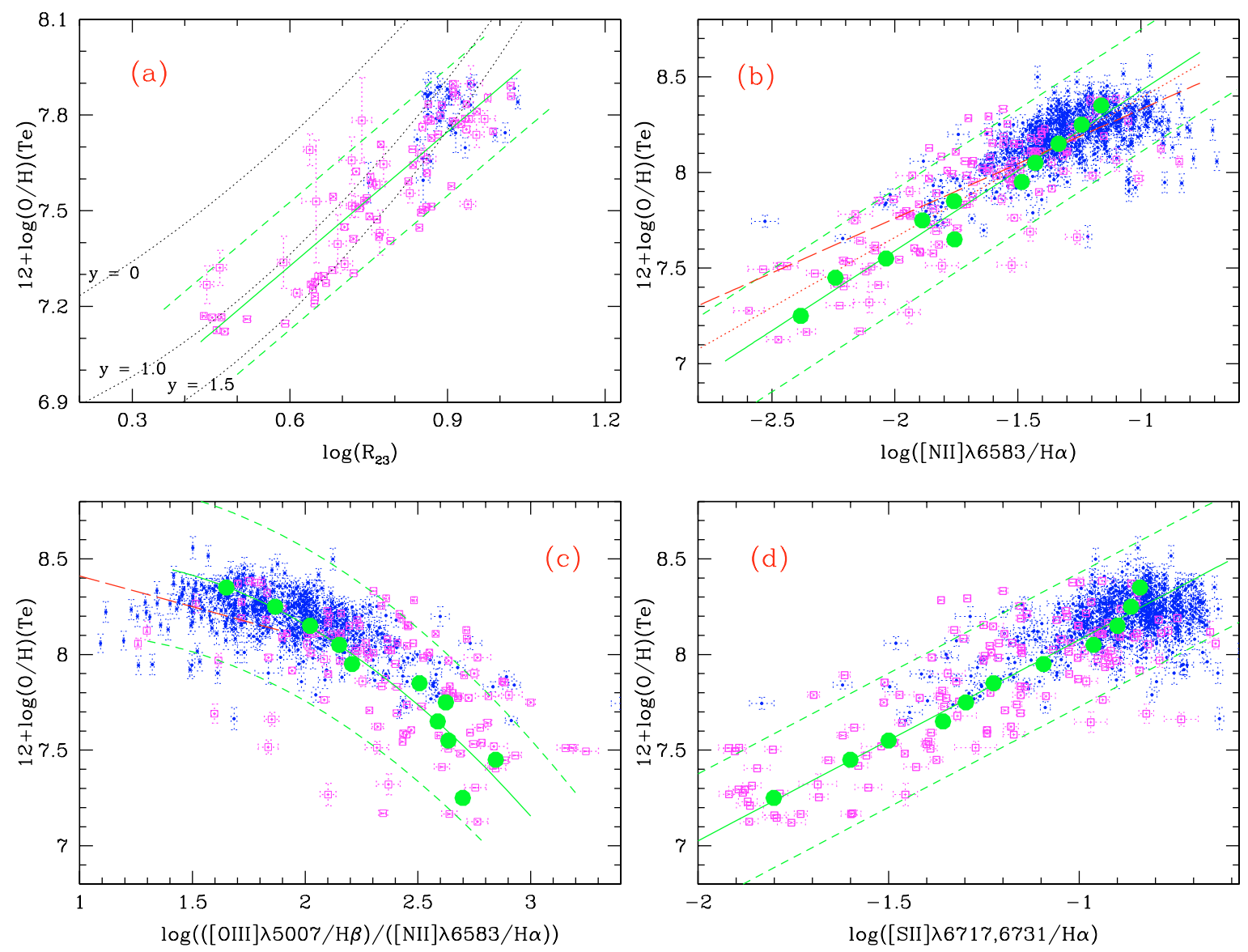

Fig. 5. $R_{23}, \mathrm{~N} 2, \mathrm{O} 3 \mathrm{~N} 2$, and $\mathrm{S} 2$ indices for oxygen abundance calibrations from the sample galaxies: a) the $R_{23}$ index; b) the $\mathrm{N} 2$ index; c) the O3N2 index; d) the S2 index. The small filled points represent the SDSS galaxies and the open squares refer to the samples from the literature. The points with uncertainties larger than $0.06 \mathrm{dex}$ in all these line ratios and $\log (\mathrm{O} / \mathrm{H})$ abundances have been excluded. The solid line a) refers to the linear least-square fit for all the 120 data points in the figure. The three dashed lines refer to the calibrations given by Kobulnicky et al. (1999) for the three different cases of $y(=\log ([\mathrm{O} \mathrm{III}] /[\mathrm{O} \mathrm{II}]))$. In $\mathbf{b})$, the long-dashed and dotted lines refer to the calibrations of PP04 and D02, respectively. In c), the long-dashed line refers to the calibration of PP04. In b)-d), the 11 large filled circles represent the mean values in each bin of oxygen abundances (see text), and the solid lines are the least-square fits for these mean-value points. The two dashed lines show the $2 \sigma$ discrepancy from the fits in all the four panels.

least-square fit for all the 120 data points with $12+\log (\mathrm{O} / \mathrm{H})<$ 7.9 is

$$
12+\log (\mathrm{O} / \mathrm{H})=6.486+1.401 \times \log \left(R_{23}\right),
$$

with $R_{23}=([\mathrm{O}$ II $] \lambda 3727+[\mathrm{O}$ III] $\lambda \lambda 4959,5007) / \mathrm{H} \beta$. The rms of the data to the fit is about 0.103 dex. The appropriate range of this calibration is about $0.4<\log \left(R_{23}\right)<1.0$ and $7.0<$ $12+\log (\mathrm{O} / \mathrm{H})<7.9$. The calibrations of Kobulnicky et al. (1999) are also presented in the figure for comparison for the cases of $y(=\log ([\mathrm{O} \mathrm{III}] /[\mathrm{O} \mathrm{II}]))$ equal to 0,1 , and 1.5 . It shows that the $y=1.0$ and 1.5 can usually explain these observational data.

\subsection{The N2 index}

Since the data points show a relatively tight linear relationship between $\mathrm{N} 2$ and $12+\log (\mathrm{O} / \mathrm{H})$, we obtain a linear least-square fit for this relation. We use the mean-value points in 11 bins of metallicities to avoid too much weight in the high metallicity range where most of the data are distributed. The bin width is $\Delta[\log (\mathrm{O} / \mathrm{H})]=0.1$ for the galaxies in the range of $7.4<12+\log (\mathrm{O} / \mathrm{H})<8.5$, and $\Delta[\log (\mathrm{O} / \mathrm{H})]=0.3$ for the range of $7.1<12+\log (\mathrm{O} / \mathrm{H})_{T_{\mathrm{e}}}<7.4$ because of the small number of sources in this range. These bin widths are the same as for the $\mathrm{O} 3 \mathrm{~N} 2$ and $\mathrm{S} 2$ indices discussed in the consequent two subsections. The objects with higher uncertainty than 0.06 dex on $\log (\mathrm{O} / \mathrm{H})$ and $\log ([\mathrm{N} \mathrm{II}] / \mathrm{H} \alpha)$ have been excluded in calculating the mean values and calibrating, which leaves 584 data points. Nineteen galaxies from the literature cannot be included in deriving calibrations since they do not have [N II] emission-line measurements. The derived analytical relation of a linear leastsquare fit is

$$
12+\log (\mathrm{O} / \mathrm{H})=9.263+0.836 \times \mathrm{N} 2,
$$

with $\mathrm{N} 2=\log ([\mathrm{N} \mathrm{II}] \lambda 6583 / \mathrm{H} \alpha)$, shown in Fig. 5b. The rms of the data to the fit is 0.159 dex. A $2 \sigma$ discrepancy is shown by the two dashed lines. This calibration is valid in the range of $-2.5<\mathrm{N} 2<-0.5$. The calibrations of D02 $(12+\log (\mathrm{O} / \mathrm{H})=$ $9.12+0.73 \times \mathrm{N} 2)$ and PP04 $(12+\log (\mathrm{O} / \mathrm{H})=8.90+0.57 \times$ N2) are also plotted for comparison. It shows that D02's calibration is similar to ours, but the one of PP04 shows a slightly lower slope and the difference is more obvious for the low metallicity region with $12+\log (\mathrm{O} / \mathrm{H})<8.0$. The reason may be that PP04's sample does not extend to much lower metallicity with $12+\log (\mathrm{O} / \mathrm{H})<7.7$, and most of their samples have metallicities of $7.7<12+\log (\mathrm{O} / \mathrm{H})<8.5$, while the fit for their these samples results in a slightly lower slope than for ours (see Fig. 1 of D02 and Fig. 1 of PP04). 


\subsection{The $\mathrm{O} 3 \mathrm{~N} 2$ index}

Figure $5 \mathrm{c}$ shows our sample galaxies for their $(\mathrm{O} / \mathrm{H})_{T_{\mathrm{e}}}$ abundances versus $\mathrm{O} 3 \mathrm{~N} 2$ indices. Although they are quite scattered, an obvious trend still exists; i.e., $\mathrm{O} / \mathrm{H}$ increases following the decreasing $\mathrm{O} 3 \mathrm{~N} 2$ index. We tried to derive a calibration of $\mathrm{O} 3 \mathrm{~N} 2$ index for $\mathrm{O} / \mathrm{H}$ abundances from these sample galaxies. The objects with an higher uncertainty than 0.06 dex on $\log (\mathrm{O} / \mathrm{H})$ and $\mathrm{O} 3 \mathrm{~N} 2$ are excluded, which leaves 585 data points. Nineteen objects from the literature without their [N II] emission-line measurements are excluded. The mean-value points within 11 bins are obtained for the left sample galaxies. The bin widths are the same as for the N2 calibration (Sect. 6.2). A two-order polynomial fit is obtained by fitting the mean-value points

$$
12+\log (\mathrm{O} / \mathrm{H})=8.203+0.630 \times \mathrm{O} 3 \mathrm{~N} 2-0.327 \times \mathrm{O} 3 \mathrm{~N} 2^{2},
$$

with $\mathrm{O} 3 \mathrm{~N} 2=\log [([\mathrm{O} \mathrm{III}] \lambda 5007 / \mathrm{H} \beta) /([\mathrm{N}$ II $] \lambda 6583 / \mathrm{H} \alpha)]$, shown in Fig. 5c. The rms of the data to the fit is 0.199 dex. This fit is valid for $1.4<\mathrm{O} 3 \mathrm{~N} 2<3$. The $2 \sigma$ discrepancy is shown by the two dashed lines. The calibration of PP04 $(12+\log (\mathrm{O} / \mathrm{H})=$ $8.73-0.32 \times \mathrm{O} 3 \mathrm{~N} 2$ ) is only valid for $1<\mathrm{O} 3 \mathrm{~N} 2<1.9$. The calibration of PP04 shows a shallower slope than ours, which comes from their much smaller sample (65 objects) and the much narrower $\mathrm{O} 3 \mathrm{~N} 2$ range.

\subsection{The $S 2$ index}

The $\mathrm{S} 2$ index $(\log ([\mathrm{S}$ II $] \lambda \lambda 6717,6731 / \mathrm{H} \alpha))$ is also a metallicitysensitive indicator. Although it is less useful than N2 for metallicity calibration due to the stronger dependence on the ionization parameter, it is still interesting to derive a calibration from $\mathrm{S} 2$ since it is dependent on metallicity monotonicly in the studied metallicity range. This relation has the advantage of not being strongly dependent on reddening corrections. Although the sample data is very scattered, a linear relation obviously exists between $\mathrm{O} / \mathrm{H}$ and $\mathrm{S} 2$.

We calculated the mean values of $\mathrm{S} 2$ and $\mathrm{O} / \mathrm{H}$ abundances for the sample galaxies in 11 bins, the bin widths are the same as we used for the N2 and O3N2 calibrations. Again, the objects with a higher uncertainty than $0.06 \operatorname{dex}$ on $\log (\mathrm{O} / \mathrm{H})$ and $\log ([\mathrm{S} \mathrm{II}] / \mathrm{H} \alpha)$ are excluded in the calculations, which leaves 607 data points. A least-square linear fit to the mean-valued points yields the relation:

$12+\log (\mathrm{O} / \mathrm{H})=9.128+1.051 \times \mathrm{S} 2$,

with $\mathrm{S} 2=\log ([\mathrm{S}$ II $] \lambda \lambda 6717,6731 / \mathrm{H} \alpha)$, shown in Fig. 5d. The rms of the data to the fit is about 0.176 dex. The $2 \sigma$ discrepancy is shown by the two dashed lines.

\subsection{Add the $P$ parameter}

The calibration of $\mathrm{O} / \mathrm{H}$ vs. $\mathrm{N} 2$ is dependent on the ionization parameter (Fig. 4b), as well the O3N2 and S2 indices (Figs. 4c, d). Therefore, to get more reliable $\mathrm{O} / \mathrm{H}$ abundances, an excitation parameter could be included in the calibrations, which correlates strongly with ionization parameter and the hardness of the ionizing radiation field. We use the $P$ parameter given by Pilyugin (2000, 2001a,b), where $P$ was defined as $[\mathrm{O} \mathrm{III}] /([\mathrm{O}$ II $]+[\mathrm{O}$ III $]$ ), which is similar to [O III]/[O II], the excitation parameter used by Kobulnicky et al. (1999) in calibrations. We should notice that $P$ needs to be computed from the dereddened emission lines.

In Fig. 6a, we plot the $\left(\log (\mathrm{O} / \mathrm{H})_{T_{\mathrm{e}}}-\log (\mathrm{O} / \mathrm{H})_{N 2}\right)$ vs. $P$ relations for the sample galaxies, which shows that these galaxies
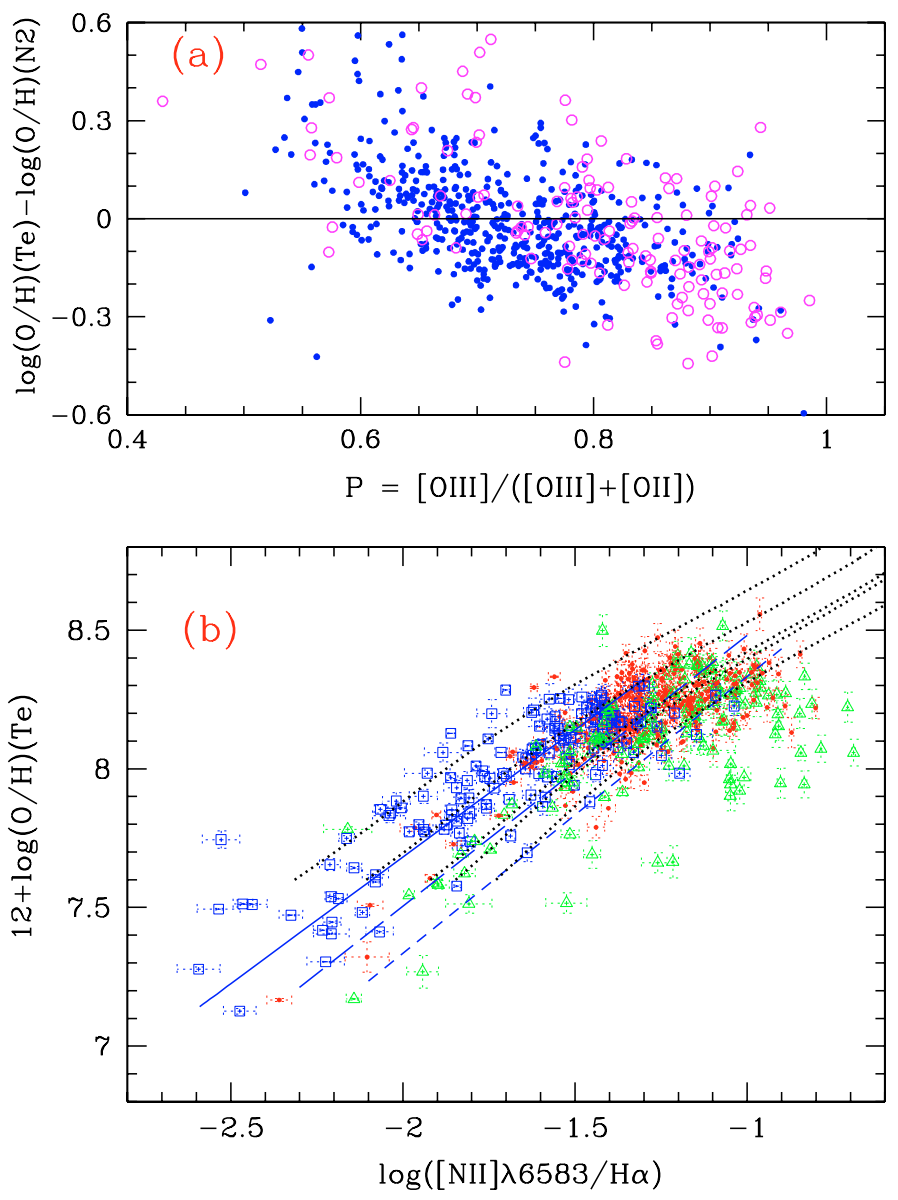

Fig. 6. a) The relation between $\log (\mathrm{O} / \mathrm{H})_{T_{\mathrm{e}}}-\log (\mathrm{O} / \mathrm{H})_{N 2}$ vs. $P$ for the sample galaxies, which shows that they can be roughly divided into three subsamples with different $P$ parameters: $P>0.80,0.65<P<$ 0.80 and $P<0.65$. The symbols are the same as in Fig. 4 . b) The calibration of $\mathrm{N} 2$ index together with the $P$ parameter. The blue open squares represent the samples with $P>0.80$, the red points represent those with $0.65<P<0.80$, and the green triangles represent those with $P<0.65$. The solid, long-dashed, and short-dashed lines refer to the linear least-square fits for each of the three subsamples, respectively (Eq. (12)). The five dotted lines are the model results of KD02, from the right to the left ones, the ionization parameter $q$ increases from the $5 \times 10^{6}$ to the $1 \times 10^{7}, 2 \times 10^{7}, 4 \times 10^{7}$, and $8 \times 10^{7} \mathrm{~cm} \mathrm{~s}^{-1}$ grids.

can be roughly separated into three subsamples with $P<0.65$ (143 data points), $0.65<P<0.80$ (292 data points), and $P>0.80$ (149 data points). We derive the N2 calibrations for these three subsamples individually. The analytical calibrations of linear least-square fits for the three subsamples with different $P$ parameters are:

$$
\begin{aligned}
12+\log (\mathrm{O} / \mathrm{H}) & =9.514+0.916 \times \mathrm{N} 2, & & (P>0.80), \\
& =9.457+0.976 \times \mathrm{N} 2, & & (0.65<P<0.80), \\
& =9.332+0.998 \times \mathrm{N} 2, & & (P<0.65) .
\end{aligned}
$$

The rms of the data to each fit are $0.150,0.140$, and $0.189 \mathrm{dex}$, respectively. The dotted lines are the photoionization model results of KD02. It shows that the three different $P$ values only correspond to the cases of KD02 of $q=4 \times 10^{7}, 1 \times 10^{7}$ (as well $2 \times 10^{7}$ ), and $5 \times 10^{6} \mathrm{~cm} \mathrm{~s}^{-1}$.

We also tried to add $P$ as an additional parameter for the O3N2 and S2 indices. The sample galaxies are separated into three sub-samples with $P<0.60,0.60<P<0.85$, and $P>0.85$ to derive calibrations for these two indices. The basic 

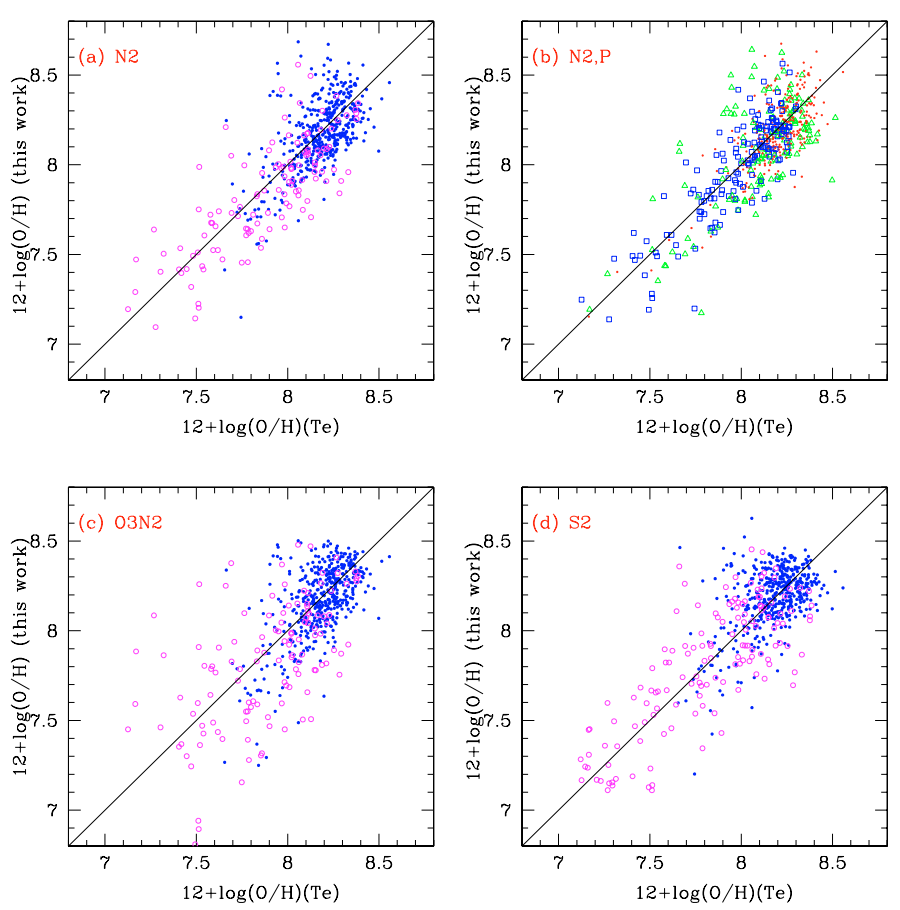

Fig. 7. Comparisons between the $T_{\mathrm{e}}$-based $\mathrm{O} / \mathrm{H}$ abundances with those derived from strong-line ratios, including $\mathrm{N} 2$ (without and with $P$ ), O3N2, and S2 indices: a) for the N2 index (without $P$, Eq. (9), Fig. 5b); b) for the N2 index (with $P$, Eq. (12), Fig. 6); c) for the O3N2 index (Eq. (10), Fig. 5c); d) for the S2 index (Eq. (11), Fig. 5d). The rms values of the data to the equal-value lines in each plot are $0.162,0.158$, 0.191 , and $0.171 \mathrm{dex}$, respectively, and they will increase to 0.173 , $0.167,0.215$, and $0.203 \mathrm{dex}$ if the data points with uncertainties larger than 0.06 dex in line indices and $\log (\mathrm{O} / \mathrm{H})$ are included. The symbols in a), c), d) are the same as in Fig. 4, and the symbols in b) are the same as in Fig. 6b.

results consistently follow the photoionization models of KD02. However, we find adding a $P$ parameter does not improve the fits much. Therefore, we do not present the two-parameter calibrations for the $\mathrm{O} 3 \mathrm{~N} 2$ and $\mathrm{S} 2$ indices as figures here. We should note that using $P$ with $[\mathrm{N} \mathrm{II}] / \mathrm{H} \alpha$ means that we need observations covering a much wider wavelength range, which might not be practical at high $z$. It is not necessary to add the $P$ parameter to the $R_{23}$ calibration since the observational data in the $R_{23}$ vs. $\mathrm{O} / \mathrm{H}$ relation does not show obvious dependence on ionization parameter in contrast to the lower-branch models (Fig. 4a).

\subsection{The accuracies of the derived calibrations}

We have presented the least-square fits for the relations of 12 $+\log (\mathrm{O} / \mathrm{H})_{T_{\mathrm{e}}}$ and $N 2, O 3 N 2$, and $S 2$ indices with and without considering the $P$ excitation parameter. To show the accuracies of these calibrations, in Figs. 7a-d we plot the comparisons between $\log (\mathrm{O} / \mathrm{H})_{T_{\mathrm{e}}}$ and those from line indices. Figures $7 \mathrm{a}, \mathrm{c}, \mathrm{d}$ show the comparisons with the calibrated abundances from N2, O3N2, S2 indices following Eqs. (9)-(11), which have been given as the solid lines in Figs. 5b-d, respectively. Figure $7 \mathrm{~b}$ shows the calibrated abundances from the N2 index but adding the $P$ parameter following Eq. (12), which improved the N2 calibration. Among these, the rms of the O3N2 and S2 calibrations are large, about 0.191 and 0.171 dex respectively (Fig. $7 \mathrm{c}, \mathrm{d}$ ). The calibration of $(\mathrm{N} 2, P)$ case (Fig. 7b) shows the lowest rms, which is about 0.158 , a bit lower than the rms in Figs. 7a and 3e for the N2 calibration (0.162 and 0.160 dex respectively).

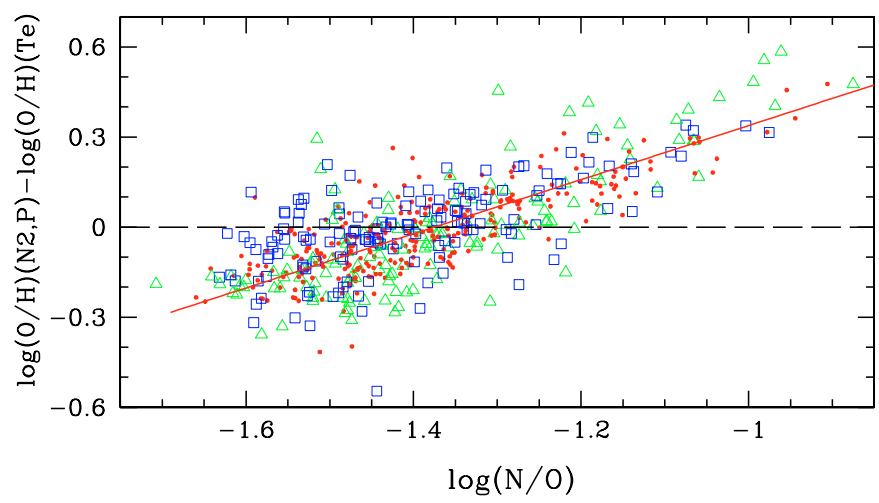

Fig. 8. Correlation between the difference in $\log (\mathrm{O} / \mathrm{H})_{N 2, P}$ and $\log (\mathrm{O} / \mathrm{H})_{T_{\mathrm{e}}}$ and the $\log (\mathrm{N} / \mathrm{O})$ abundance ratios of the sample galaxies. The symbols are the same as in Figs. 6b, 7b. The solid line is the linear least-square fit for the relations with an rms of 0.104 dex.

Some of the remaining scatter in $\log (\mathrm{O} / \mathrm{H})_{N 2, P}$ may come from the different star-forming history and evolutionary status of the sample galaxies. Figure 8 shows a clear correlation between the $\log (\mathrm{O} / \mathrm{H})_{N 2, P}-\log (\mathrm{O} / \mathrm{H})_{T_{\mathrm{e}}}$ and the $\log (\mathrm{N} / \mathrm{O})$ abundance ratios of the sample galaxies, which can be given as a linear leastsquare fit:

$\log (\mathrm{O} / \mathrm{H})_{N 2, P}-\log (\mathrm{O} / \mathrm{H})_{T_{\mathrm{e}}}=0.902 \times \log (\mathrm{N} / \mathrm{O})+1.240$,

with an rms of 0.104 dex. The corrected calibration of $(N 2, P)$ by considering this correlation will provide more accurate oxygen abundance, and the rms of the data to the equal-value lines in Figs. $7 \mathrm{a}$ and $7 \mathrm{~b}$ will decrease to $0.115 \mathrm{dex}$, which is much less than the previous ones ( 0.162 and 0.158 dex, respectively). This correlation can be used to correct the derived oxygen abundances from the strong-line ratio when it is necessary. Nevertheless, to apply the $\mathrm{N} / \mathrm{O}$ correction to $12+\log (\mathrm{O} / \mathrm{H})$ abundances, we need to measure [O II], [O III], and $\mathrm{H} \beta$, in addition to [N II] and $\mathrm{H} \alpha$, so it will not always be practical.

We should notice that both the $x$ and $y$ axes of Fig. 8 depend on nitrogen abundance, which means that the observed correlation could be spurious if the errors in $\log (\mathrm{N})$ are large. However, the errors in the $\log (\mathrm{N} / \mathrm{O})$ abundances of the sample galaxies are quite small. It is about 0.011 dex on average for the MPA/JHU sample galaxies, which is expected since we only select the objects with higher signal-to-noise ratio, i.e. larger than $5 \sigma$, on the flux measurements of [N II] (see Sect. 2.1). For other samples from the literature, the errors in their $\log (\mathrm{N} / \mathrm{O})$ values are about $0.021 \mathrm{dex}$ on average. Therefore, this correlation is robustly measured and could be used to correct $12+\log (\mathrm{O} / \mathrm{H})_{\mathrm{N} 2, \mathrm{P}}$ measurements if desired. More important, the strong trend in Fig. 8 highlights the sensitivity of the N2 calibration to a galaxy's past history of star formation, since it determines the present-day N/O ratio.

\section{Conclusions}

We compiled a large sample of 695 low-metallicity galaxies and $\mathrm{H}$ II regions with [O III]4363 detected, which consists of 531 galaxies from the SDSS-DR4 and 164 galaxies and $\mathrm{H}$ II regions from the literature. We determined their electron temperatures, electron densities, and then the $T_{\mathrm{e}}$-based oxygen abundances. The derived oxygen abundances for them are $7.1<$ $12+\log (\mathrm{O} / \mathrm{H})<8.5$. 
The comparison of the $T_{\mathrm{e}}$-based oxygen abundances and the Bayesian metallicities obtained by the MPA/JHU group show that, for about half of the MPA/JHU sample galaxies in this study, their $\mathrm{O} / \mathrm{H}$ abundances were overestimated by a factor of 0.34 dex on average by using the photoionization models (Charlot et al. 2006; Tremonti et al. 2004; Brinchmann et al. 2004). This is possibly related to how secondary nitrogen enrichment is treated in the models they used.

The $T_{\mathrm{e}}$-derived oxygen abundances of these sample galaxies were compared with those derived from other strong-line ratios, such as $R_{23}, R_{23}-P$, N2, and O3N2 indicators. The results show that the $R_{23}$ and $R_{23}-P$ methods will generally overestimate the oxygen abundances of the sample galaxies by a factor of $\sim 0.20$ dex and $\sim 0.06$ dex, respectively. This 0.06 dex discrepancy is quite small, and will decrease to 0.025 dex when the improved $R_{23}-P$ calibrations given by Pilyugin \& Thuan (2005) are used. The abundance calibration of $\mathrm{N} 2$ index, rather than $\mathrm{O} 3 \mathrm{~N} 2$ index, from $\mathrm{PP} 04$ can result in consistent $\mathrm{O} / \mathrm{H}$ abundances with $(\mathrm{O} / \mathrm{H})_{T_{\mathrm{e}}}$ but with a scatter of about $0.16 \mathrm{dex}$.

From this large sample of 695 star-forming galaxies and $\mathrm{H} \mathrm{II}$ regions, we re-derive abundance calibrations for $R_{23}, \mathrm{~N} 2, \mathrm{O} 3 \mathrm{~N} 2$, and $\mathrm{S} 2$ indices on the basis of their oxygen abundances derived from $T_{\mathrm{e}}$. The N2, O3N2, and $\mathrm{S} 2$ indices monotonicly change following the increasing $\mathrm{O} / \mathrm{H}$ abundances from $12+\log (\mathrm{O} / \mathrm{H})=7.1$ to 8.5 , which are consistent with the photoionization model results of KD02. The $R_{23}$ parameter is a good metallicity indicator for the metal-poor galaxies with $12+\log (\mathrm{O} / \mathrm{H})<7.9$. Nevertheless, $R_{23}$ does not follow the KD02 models well.

The scatter of the observational data may come from the differences in the ionizing radiation field. We add an excitation parameter $P$ to separate the sample galaxies into three sub-samples, and then re-derive the $\mathrm{N} 2$ calibrations with three different $P$ cases $(<0.65,0.65-0.80,>0.80)$, which improves the calibration, confirmed by the decreasing rms of the data to the equal-value line, which decreases from 0.162 in Fig. 7 a to 0.158 in Fig. $7 b$. Adding the $P$ parameter in the $\mathrm{O} 3 \mathrm{~N} 2$ and $\mathrm{S} 2$ indices does not improve the calibrations. However, some of the remaining scatter in the $(\mathrm{N} 2, \mathrm{P})$ calibration may come from the different starforming history and evolutionary status of the galaxies, which can be confirmed by the correlation between the $\log (\mathrm{O} / \mathrm{H})_{N 2, P}-$ $\log (\mathrm{O} / \mathrm{H})_{T_{\mathrm{e}}}$ and $\log (\mathrm{N} / \mathrm{O})$ of them (Fig. 8 and Eq. (13)).

In future studies of the calibrating oxygen abundances of galaxies, when their $T_{\mathrm{e}}$ values are not available, we can use the $R_{23}, \mathrm{~N} 2, \mathrm{O} 3 \mathrm{~N} 2$, or $\mathrm{S} 2$ indices, together with the $P$ parameter if the wavelength coverage is wide enough, to calibrate their oxygen abundances. The $R_{23}$ parameter shows obvious correlation with oxygen abundances for the low-metallicity galaxies with $12+\log (\mathrm{O} / \mathrm{H})<7.9$. Compared with $\mathrm{O} 3 \mathrm{~N} 2$ and $\mathrm{S} 2$, The N2 index is less affected by ionization parameter and more obviously correlated with $\mathrm{O} / \mathrm{H}$ abundances. Also it can be detected by the near-infrared instruments for the intermediate- and high- $z$ star-forming galaxies. The N2 index has helped to provide important information on the metallicities of galaxies at high- $z$; for example, see Shapley et al. (2004) for a sample of 7 starforming galaxies at $2.1<z<2.5$, Shapley et al. (2005) for a sample of 12 star-forming galaxies at $z \sim 1.0-1.5$, and Erb et al. (2006) for the six composited spectra of 87 rest-frame UVselected star-forming galaxies at $z>2$, etc. However, considering the dependence of $\mathrm{N} 2$, as well as the $(\mathrm{N} 2, \mathrm{P})$ calibration on $\log (\mathrm{N} / \mathrm{O})$ shown as Fig. 8, we should keep in mind that the resulting $\log (\mathrm{O} / \mathrm{H})$ abundances from $\mathrm{N} 2$ may involve the specific history of $\mathrm{N}$-enrichment in the galaxies. We may worry a bit about comparing the low and high- $z$ observations using [N II] for this reason.
Given the good consistency of our N2 and S2 abundance calibrations with the models of KD02 at low metallicity (see Fig. 4b,d), it may be appropriate to linearly extrapolate our calibrations up to the high metallicity region, up to $12+\log (\mathrm{O} / \mathrm{H}) \sim 9.0$ and 8.8 respectively. However, this extrapolation on $\mathrm{N} 2$ will result in a $\operatorname{lower} \log (\mathrm{O} / \mathrm{H})$ abundance than the one directly derived from the calibration of the metal-rich SDSS galaxies obtained by Liang et al. (2006), which may be due to the discrepancy between the $T_{\mathrm{e}}$ - and the $R_{23}$-based metallicities. However, the $T_{\mathrm{e}}$-based $\mathrm{O} 3 \mathrm{~N} 2$ calibration cannot be directly extrapolated to the metal-rich region since the slopes of the correlation relations between $\log (\mathrm{O} / \mathrm{H})$ vs. $\mathrm{O} 3 \mathrm{~N} 2$ are not the same in the high-metallicity branch as in the low-metallicity branch (Fig. 4c). The $R_{23}$ parameter cannot provide reliable oxygen abundances for the galaxies in the metallicity turn-over region with $7.9<12+\log (\mathrm{O} / \mathrm{H})<8.4$.

Acknowledgements. We thank the referee for many valuable and wise suggestions and comments, which helped us in improving this work. We thank Stephane Charlot for the interesting discussions about their models for estimating the oxygen abundances of the SDSS galaxies. S.Y.Y. and Y.C.L. thank Jing Wang and Caina Hao for the interesting discussions about the SDSS database. S.Y.Y thanks Yongheng Zhao and the LAMOST group for kindly providing their office for his stay. This work was supported by the Natural Science Foundation of China (NSFC) Foundation under No.10403006, 1043301010373005,10573022 , 10333060 , and 10521001 .

\section{References}

Adelman-McCarthy, J. K., et al. 2006, ApJS, 162, 38

Alloin, D., Collin-Souffrin, S., Joly, M., \& Vigroux, L., 1979, A\&A, 78, 200

Baldwin, J., Phillips, M. M., \& Terlevich, R. J. 1981, PASP, 93, 5

Bresolin, F., Garnett, D. R., \& Kennicutt, R. C. Jr. 2004, ApJ, 615, 228

Bresolin, F., Schaerer, D. et al. 2005, A\&A, 441, 981

Brinchmann, J., Charlot, S., White, S. D. M., et al. 2004 MNRAS, 351, 1151

Bruzual, A. G., \& Charlot, S. 2003, MNRAS, 344, 1000

Calzetti, D., Kinney, A. L., \& Storchi-Bergmann, T. 1994, ApJ, 429, 582

Charlot, S., \& Longhetti, M. 2001, MNRAS, 323, 887 (CL01)

de Robertis, M. M., Dufour, R. J., \& Hunt, R. W. 1987, JRASC, 81, 195

Denicoló, G., Terlevich, R., \& Terlevich, E. 2002, MNRAS, 330, 69 (D02)

Díaz, A. I., Terlevich, E., Vílchez, J. M., Pagel, B. E., \& Edmunds, M. G., 1991, MNRAS, 253, 245

Erb, D. K., Shapley, A. E., Pettini, M., et al. 2006, ApJ, 644, 813

Garnett, D. R. 1992, AJ, 103, 1330

Garnett, D. R., Edmunds, M. G., Henry, R. B. C., Pagel, B. E. J., \& Skillman, E. D. 2004a, AJ, 128, 2772

Garnett, D. R., Kennicutt, R. C. Jr., \& Bresolin, F. 2004b, ApJ, 607, 21 Guseva, N. G., Papaderos, P., Izotov, Y. I., et al. 2003a, A\&A, 407, 75 Guseva, N. G., Papaderos, P., Izotov, Y. I., et al. 2003b, A\&A, 407, 91 Guseva, N. G., Papaderos, P., Izotov, Y. I., et al. 2003c, A\&A, 407, 105 Izotov, Y. I., \& Thuan, T. X. 1998a, ApJ, 497, 227

Izotov, Y. I., \& Thuan, T. X. 1998b, ApJ, 500, 188 Izotov, Y. I., \& Thuan, T. X. 1999, ApJ, 511, 639 Izotov, Y. I., \& Thuan, T. X. 2004, ApJ, 602, 200

Izotov, Y. I., Thuan, T. X., \& Lipovetsky, V. A., 1994, ApJ, 435, 647 Izotov, Y. I., Andrew, B. D., et al. 1996, ApJ, 458, 524

Izotov, Y. I., Thuan, T. X., \& Lipovetsky, V. A. 1997a, ApJS, 108, 1

Izotov, Y. I., Lipovetsky, V. A., et al. 1997b, ApJ, 476, 698

Izotov, Y. I., Chaffee, F. H., Foltz, R. F., et al., T. X., 1999, ApJ, 527, 757

Izotov, Y. I., Chaffee, F. H., \& Green, R. F. 2001a, ApJ, 562, 727

Izotov, Y. I., Chaffee, F. H., \& Schaerer, D. 2001b, A\&A, 378, 45

Izotov, Y. I., Papaderos, P., Guseva, N. G., Fricke, K. J., \& Thuan, T. X., 2004, A\&A, 421, 539

Izotov, Y. I., Stasinska, G., Meynet, G., Guseva, N. G., \& Thuan, T. X. 2006, A\&A, 448, 955

Kauffmann, G., Heckman, T. M., Tremonti, C., et al. 2003, MNRAS, 346, 1055 Kennicutt, R. C. Jr., 1992, ApJ, 388, 310

Kennicutt, R. C. Jr., Bresolin, F., \& Garnett, D. R. 2003, ApJ, 591, 801

Kewley, L. J., \& Dopita, M. A. 2002, ApJS, 142, 35 (KD02)

Kewley, L. J., Dopita, M. A., Sutherland, R. S., Heisler C. A., \& Trevena, J. 2001, ApJ, 556, 121

Kewley, L. J., Jansen, R. A., \& Geller, M. J. 2005, PASP, 117, 227 
Kinney, A. L., Bohlin, R. C., Calzetti, D., Panagia, N., \& Wyse, R. F. G., 1993, ApJS, 86, 5

Kniazev, A. Y., Pustilnik, S. A., Masegosa, J., et al. 2000, A\&A, 357, 101

Kobulnicky, H. A., Kennicutt, R. C. Jr., \& Pizagno, J. L. 1999, ApJ, 514, 544 (K99)

Lamareille, F., Contini, T., Brinchmann, J., et al. 2006, A\&A, 448, 907

Lee, J. C., Salzer, J. J., \& Melbourne, J. 2004, ApJ, 616, 752

Liang, Y. C., Yin, S. Y., Hammer, F., et al. 2006, ApJ, 652, 257

McGaugh, S. S. 1991, ApJ, 380, 140

Melbourne, J., Phillips, A., Salzer, J. J., Gronwall, C., \& Sarajedini, V. 1. 2004, AJ, 127, 686

Moustakas, J., \& Kennicutt, R. C.Jr. 2006, ApJ, 651, 155

Nagao, T., Maiolino, R., \& Marconi, A. 2006, A\&A, 459, 85

Osterbrock, D. E. 1989, Astrophysics of Gaseous Nebulae and Active Galactic Nuclei (Mill Valley, California: University Science Books)

Pagel, B. E. J., Edmunds, M. G., Blackwell, D. E., et al. 1979, MNRAS, 189, 95 Pettini, M. \& Pagel, B. E. J. 2004, MNRAS, 348, L59 (PP04)

Pérez-Montero, E., \& Díaz A. I. 2005, MNRAS, 361, 1063

Pilyugin, L. S. 2000, A\&A, 362, 325

Pilyugin, L. S. 2001a, A\&A, 369, 594

Pilyugin, L. S. 2001b, A\&A, 373, 56

Pilyugin, L. S., \& Thuan, T. X. 2005, ApJ, 631, 231
Pilyugin, L. S. Contini, T., Vílchez, J. M. 2004, A\&A, 423, 427

Pilyugin, L. S., Thuan, T. X., \& Vílchez, J. M. 2006, MNRAS, 367, 1139

Raimann, D., Storchi-Bergmann, T., Bica, E., et al. 2000, MNRAS, 316, 559

Shapley, A. E., Erb, D. K., Pettini, M., et al. 2004, ApJ, 612, 108

Shapley, A. E., Coil, A. L., Ma, C. P., \& Bundy, K. 2005, ApJ, 635, 1006

Shaw, R. A., \& Dufour, R. J., 1995, PASP, 107, 896

Shi, F., Kong, X., Li, C., \& Cheng, F. Z. 2005, A\&A, 437, 849

Shi, F., Kong, X., \& Cheng, F. Z. 2006, A\&A, 453, 487

Skillman, E. D., Kennicutt, R. C. Jr., \& Hodge, P. W., 1989, ApJ, 347, 875

Skillman, E. D., Cote, S., \& Miller, B. W. 2003, AJ, 125, 610

Stasińska, G. 2002, Lectures to be published in the proceedings of the XIII Canary Islands Winter School of Astrophysics, [arXiv: astro-ph/0207500]

Stasińska, G. 2005, A\&A, 434, 507

Stasińska, G., \& Leitherer C. 1996, ApJS, 107, 661

Storchi-Bergmann, T., Calzetti D., \& Kinney A. L. 1994, ApJ, 429, 572

Thurston, T. R., Edmunds, M. G., \& Henry, R. B. C. 1996, MNRAS, 283, 990

Tremonti, C. A., Heckman, T. M., Kauffmann, G., et al. 2004, ApJ, 613, 898 van Zee, L. 2000, ApJ, 543, L31

van Zee, L., \& Haynes, M. P. 2006, ApJ, 636, 214

Veilleux, S., \& Osterbrock, D. 1987, ApJS, 63, 295

Vílchez, J. M., \& Iglesias-Páramo, J. 2003, ApJS, 145, 225 
S. Y. Yin et al.: Empirical strong-line oxygen abundance calibrations from galaxies with electron-temperature measurements, Online Material p 1

\section{Online Material}


Table 1. The characteristic parameters of the SDSS galaxies: $\mathrm{N} 2=\log ([\mathrm{N}$ II $] 6483 / \mathrm{H} \alpha), \mathrm{O} 3 \mathrm{~N} 2=\log (([\mathrm{O} \mathrm{III}] 5007 / \mathrm{H} \beta) /([\mathrm{N}$ II $] 6483 / \mathrm{H} \alpha))$, $\mathrm{S} 2=\log ([\mathrm{S} \mathrm{II}] 6717,6731 / \mathrm{H} \alpha), P=[\mathrm{O} \mathrm{III}] /([\mathrm{O} \mathrm{II}]+[\mathrm{O} \mathrm{III}]) .(\mathrm{O} / \mathrm{H})_{\text {Bay }}=12+(\mathrm{O} / \mathrm{H})_{\text {Bayesian }}$ obtained by the MPA/JHU group.

\begin{tabular}{|c|c|c|c|c|c|c|c|c|c|}
\hline (1) & (2) & (3) & (4) & (5) & (6) & (7) & (8) & (9) & (10) \\
\hline Num & Plate-MJD-FiberID & IAU designations & $t_{3}\left(10^{4} \mathrm{~K}\right)$ & $12+(\mathrm{O} / \mathrm{H})_{T_{\mathrm{e}}}$ & $(\mathrm{O} / \mathrm{H})_{\text {Bay }}$ & $\mathrm{N} 2$ & S2 & $\mathrm{O} 3 \mathrm{~N} 2$ & $P$ \\
\hline 1 & $266-51630-407$ & J094333.91+010659.4 & $1.080 \pm 0.062$ & $8.197 \pm 0.050$ & 8.659 & $-0.911 \pm 0.005$ & $-0.683 \pm 0.005$ & $1.436 \pm 0.003$ & 0.552 \\
\hline 2 & 270- 51909- 617 & J101430.99+004754.9 & $1.198 \pm 0.037$ & $8.141 \pm 0.031$ & 8.427 & $-1.331 \pm 0.010$ & $-0.954 \pm 0.011$ & $2.128 \pm 0.008$ & 0.776 \\
\hline 3 & 276- 51909- 490 & $\mathrm{~J} 105342.55+000945.0$ & $1.203 \pm 0.073$ & $8.142 \pm 0.051$ & 8.179 & $-1.250 \pm 0.014$ & $-0.746 \pm 0.009$ & $1.941 \pm 0.012$ & 0.639 \\
\hline 4 & 277- 51908- 451 & J110116.39+004814.4 & $1.450 \pm 0.029$ & $7.982 \pm 0.025$ & 8.090 & $-1.605 \pm 0.026$ & $-1.346 \pm 0.029$ & $2.581 \pm 0.024$ & 0.924 \\
\hline 5 & $282-51658-543$ & J113703.79+002817.4 & $1.199 \pm 0.025$ & $8.210 \pm 0.022$ & 8.206 & $-1.413 \pm 0.014$ & $-0.918 \pm 0.008$ & $2.303 \pm 0.012$ & 0.811 \\
\hline 6 & 283- 51959- 572 & J114649.34+005345.9 & $1.608 \pm 0.035$ & $7.884 \pm 0.030$ & 7.990 & $-2.019 \pm 0.041$ & $-1.392 \pm 0.022$ & $3.018 \pm 0.033$ & 0.937 \\
\hline 7 & 285- 51930- 154 & J120047.07-003611.8 & $1.056 \pm 0.067$ & $8.311 \pm 0.061$ & 8.234 & $-1.247 \pm 0.010$ & $-0.769 \pm 0.009$ & $1.947 \pm 0.008$ & 0.656 \\
\hline 8 & 289- 51990- 369 & J122419.73+010559.6 & $1.245 \pm 0.033$ & $8.075 \pm 0.027$ & 7.993 & $-1.615 \pm 0.014$ & $-1.019 \pm 0.009$ & $2.419 \pm 0.012$ & 0.809 \\
\hline 9 & 292- 51609- 372 & $\mathrm{~J} 124715.31+003806.7$ & $1.235 \pm 0.048$ & $8.099 \pm 0.032$ & 8.087 & $-1.362 \pm 0.011$ & $-0.774 \pm 0.007$ & $2.077 \pm 0.009$ & 0.679 \\
\hline 10 & 296- 51984- 416 & J131937.25+005043.8 & $1.005 \pm 0.028$ & $8.417 \pm 0.032$ & 8.469 & $-1.352 \pm 0.008$ & $-0.966 \pm 0.007$ & $2.184 \pm 0.005$ & 0.774 \\
\hline 11 & 297- 51959- 442 & $\mathrm{~J} 132654.62+011346.5$ & $1.175 \pm 0.028$ & $8.178 \pm 0.026$ & 8.348 & $-1.434 \pm 0.013$ & $-1.041 \pm 0.013$ & $2.278 \pm 0.011$ & 0.822 \\
\hline 12 & 299- 51671- 311 & J133649.51-001158.9 & $0.977 \pm 0.040$ & $8.381 \pm 0.045$ & 8.615 & $-1.222 \pm 0.008$ & $-0.911 \pm 0.007$ & $1.945 \pm 0.005$ & 0.731 \\
\hline 13 & 304- 51957- 12 & J142214.30-003919.8 & $1.411 \pm 0.044$ & $7.971 \pm 0.028$ & 8.076 & $-1.580 \pm 0.026$ & $-1.126 \pm 0.014$ & $2.426 \pm 0.024$ & 0.804 \\
\hline 14 & 304- 51957- 568 & $\mathrm{~J} 142200.19+010213.2$ & $0.994 \pm 0.056$ & $8.400 \pm 0.057$ & 8.448 & $-1.225 \pm 0.008$ & $-0.812 \pm 0.011$ & $1.938 \pm 0.006$ & 0.667 \\
\hline 15 & $308-51662-412$ & $\mathrm{~J} 144458.53+004618.8$ & $0.972 \pm 0.048$ & $8.432 \pm 0.054$ & 8.706 & $-1.009 \pm 0.008$ & $-0.887 \pm 0.011$ & $1.796 \pm 0.007$ & 0.758 \\
\hline 16 & 309-51994- 489 & J145533.67+003657.2 & $1.126 \pm 0.053$ & $8.232 \pm 0.039$ & 8.577 & $-1.006 \pm 0.006$ & $-0.727 \pm 0.007$ & $1.678 \pm 0.004$ & 0.617 \\
\hline 17 & $310-51990-136$ & J150356.83-004200.3 & $1.059 \pm 0.045$ & $8.308 \pm 0.043$ & 8.358 & $-1.300 \pm 0.010$ & $-0.866 \pm 0.008$ & $2.053 \pm 0.008$ & 0.720 \\
\hline 18 & $311-51665-8$ & $\mathrm{~J} 151320.62-002551.9$ & $1.119 \pm 0.064$ & $8.130 \pm 0.054$ & 8.842 & $-0.871 \pm 0.007$ & $-0.918 \pm 0.012$ & $1.517 \pm 0.005$ & 0.711 \\
\hline 19 & 313- 51673- 638 & $\mathrm{~J} 152830.72+001740.2$ & $1.185 \pm 0.039$ & $8.150 \pm 0.032$ & 8.454 & $-1.385 \pm 0.013$ & $-0.906 \pm 0.009$ & $2.172 \pm 0.010$ & 0.770 \\
\hline 20 & $337-51997-593$ & J125526.06-021333.9 & $1.560 \pm 0.029$ & $7.831 \pm 0.026$ & 7.854 & $-2.040 \pm 0.048$ & $-1.497 \pm 0.018$ & $2.958 \pm 0.044$ & 0.942 \\
\hline 21 & $339-51692-437$ & $\mathrm{~J} 130324.17-021046.9$ & $0.982 \pm 0.053$ & $8.372 \pm 0.056$ & 8.493 & $-1.141 \pm 0.007$ & $-0.741 \pm 0.006$ & $1.814 \pm 0.005$ & 0.670 \\
\hline 22 & 344- 51693- 321 & $\mathrm{~J} 155957.38+004741.2$ & $1.305 \pm 0.058$ & $8.077 \pm 0.041$ & 8.739 & $-1.375 \pm 0.022$ & $-1.115 \pm 0.029$ & $2.265 \pm 0.020$ & 0.854 \\
\hline 23 & 348- 51671- 297 & J163012.60-005408.6 & $1.157 \pm 0.045$ & $8.169 \pm 0.034$ & 8.474 & $-1.149 \pm 0.010$ & $-0.776 \pm 0.016$ & $1.856 \pm 0.008$ & 0.685 \\
\hline 24 & 348- 51671- 331 & J163107.20+005324.7 & $1.175 \pm 0.055$ & $8.159 \pm 0.042$ & 8.084 & $-1.357 \pm 0.010$ & $-0.813 \pm 0.008$ & $2.084 \pm 0.009$ & 0.698 \\
\hline 25 & $350-51691-500$ & $\mathrm{~J} 171212.07+650248.4$ & $1.137 \pm 0.065$ & $8.072 \pm 0.049$ & 8.819 & $-0.786 \pm 0.004$ & $-0.773 \pm 0.006$ & $1.320 \pm 0.002$ & 0.625 \\
\hline 26 & $351-51780-212$ & J170201.44+604746.3 & $1.003 \pm 0.040$ & $8.316 \pm 0.038$ & 8.616 & $-0.963 \pm 0.005$ & $-0.739 \pm 0.005$ & $1.579 \pm 0.003$ & 0.636 \\
\hline 27 & $351-51780-600$ & $\mathrm{~J} 170922.63+614851.1$ & $1.046 \pm 0.066$ & $8.251 \pm 0.058$ & 8.622 & $-1.029 \pm 0.008$ & $-0.705 \pm 0.009$ & $1.612 \pm 0.006$ & 0.607 \\
\hline 28 & 353- 51703- 479 & J170517.74+593546.6 & $1.127 \pm 0.063$ & $8.152 \pm 0.047$ & 8.589 & $-1.036 \pm 0.006$ & $-0.669 \pm 0.005$ & $1.618 \pm 0.005$ & 0.600 \\
\hline 29 & 358- 51818- 403 & $\mathrm{~J} 173021.82+571531.6$ & $1.074 \pm 0.061$ & $8.297 \pm 0.055$ & 8.568 & $-1.181 \pm 0.010$ & $-0.848 \pm 0.011$ & $1.942 \pm 0.008$ & 0.721 \\
\hline 30 & 363- 51989- 532 & $\mathrm{~J} 153258.27+004100.2$ & $1.036 \pm 0.065$ & $8.346 \pm 0.062$ & 8.694 & $-0.837 \pm 0.006$ & $-0.729 \pm 0.007$ & $1.558 \pm 0.003$ & 0.673 \\
\hline 31 & $364-52000-66$ & J163006.89-001810.8 & $1.106 \pm 0.063$ & $8.255 \pm 0.053$ & 8.483 & $-1.162 \pm 0.009$ & $-0.789 \pm 0.007$ & $1.898 \pm 0.007$ & 0.687 \\
\hline 32 & 364- 52000- 384 & $\mathrm{~J} 162150.66+003509.2$ & $1.337 \pm 0.079$ & $8.018 \pm 0.041$ & 8.334 & $-1.048 \pm 0.007$ & $-0.576 \pm 0.006$ & $1.647 \pm 0.005$ & 0.537 \\
\hline 33 & 374- 51791- 612 & J221823.86+003918.3 & $1.097 \pm 0.074$ & $8.260 \pm 0.065$ & 8.315 & $-1.214 \pm 0.013$ & $-0.780 \pm 0.010$ & $1.965 \pm 0.011$ & 0.714 \\
\hline 34 & 375- 52140- 118 & J222510.13-001152.8 & $1.413 \pm 0.020$ & $7.972 \pm 0.020$ & 7.984 & $-1.763 \pm 0.012$ & $-1.250 \pm 0.008$ & $2.680 \pm 0.010$ & 0.896 \\
\hline 35 & 375- 52140- 348 & J221823.86+003918.3 & $1.150 \pm 0.063$ & $8.213 \pm 0.051$ & 8.343 & $-1.213 \pm 0.012$ & $-0.787 \pm 0.009$ & $1.969 \pm 0.009$ & 0.700 \\
\hline 36 & $380-51792-253$ & J225906.70-005810.2 & $0.972 \pm 0.058$ & $8.459 \pm 0.065$ & 8.619 & $-1.131 \pm 0.007$ & $-0.931 \pm 0.008$ & $1.911 \pm 0.005$ & 0.716 \\
\hline 37 & 384- 51821- 281 & J232936.56-011057.0 & $1.184 \pm 0.025$ & $8.192 \pm 0.024$ & 8.217 & $-1.384 \pm 0.008$ & $-0.931 \pm 0.007$ & $2.249 \pm 0.006$ & 0.821 \\
\hline 38 & $386-51788-573$ & J235347.69+005402.1 & $1.194 \pm 0.088$ & $8.179 \pm 0.067$ & 8.217 & $-1.379 \pm 0.033$ & $-0.866 \pm 0.023$ & $2.182 \pm 0.031$ & 0.742 \\
\hline 39 & $388-51793-239$ & J000703.98-003447.6 & $1.246 \pm 0.033$ & $8.128 \pm 0.027$ & 8.163 & $-1.468 \pm 0$ & $-0.970 \pm 0.011$ & $2.313 \pm 0.013$ & 0.792 \\
\hline 40 & 388- 51793- 457 & J000657.02+005125.9 & $1.221 \pm 0.029$ & $8.173 \pm 0.025$ & 8.492 & $-1.345 \pm 0.009$ & $-0.989 \pm 0.009$ & $2.215 \pm 0.006$ & 0.799 \\
\hline 41 & 394- 51913- 402 & J005105.28+004600.1 & $0.982 \pm 0.049$ & $8.426 \pm 0.051$ & 8.177 & $-1.297 \pm 0.008$ & $-0.814 \pm 0.005$ & $2.034 \pm 0.007$ & 0.684 \\
\hline 42 & 398- 51789- 223 & J011633.94-002043.0 & $1.144 \pm 0.076$ & $8.187 \pm 0.063$ & 8.115 & $-1.281 \pm 0.015$ & $-0.750 \pm 0.010$ & $2.002 \pm 0.013$ & 0.699 \\
\hline 43 & 406- 51869- 484 & J022407.68+003226.1 & $1.141 \pm 0.072$ & $8.194 \pm 0.053$ & 8.104 & $-1.309 \pm 0.010$ & $-0.699 \pm 0.006$ & $1.956 \pm 0.008$ & 0.609 \\
\hline 44 & 408- 51821- 472 & J023900.79+001836.0 & $1.265 \pm 0.037$ & $8.140 \pm 0.033$ & 8.376 & $-1.509 \pm 0.028$ & $-1.145 \pm 0.027$ & $2.455 \pm 0.026$ & 0.891 \\
\hline 45 & 409- 51871- 50 & J024939.72-011151.3 & $1.309 \pm 0.033$ & $8.042 \pm 0.028$ & 8.013 & $-1.652 \pm 0.015$ & $-1.060 \pm 0.010$ & $2.519 \pm 0.013$ & 0.857 \\
\hline 46 & 414- 51901- 546 & J032750.16+010135.0 & $1.057 \pm 0.048$ & $8.296 \pm 0.038$ & 8.472 & $-1.104 \pm 0.006$ & $-0.689 \pm 0.004$ & $1.742 \pm 0.004$ & 0.601 \\
\hline 47 & $415-51810-285$ & J033031.22-005846.5 & $1.178 \pm 0.034$ & $8.199 \pm 0.031$ & 8.127 & $-1.481 \pm 0.013$ & $-0.972 \pm 0.008$ & $2.343 \pm 0.010$ & 0.815 \\
\hline 48 & 419- 51879- 364 & J004236.94+160202.7 & $1.103 \pm 0.042$ & $8.265 \pm 0.040$ & 8.814 & $-1.091 \pm 0.010$ & $-1.054 \pm 0.030$ & $1.926 \pm 0.008$ & 0.807 \\
\hline 49 & $420-51871-156$ & J005425.68+144852.2 & $1.156 \pm 0.035$ & $8.206 \pm 0.029$ & 8.160 & $-1.406 \pm 0.009$ & $-0.929 \pm 0.007$ & $2.190 \pm 0.007$ & 0.737 \\
\hline 50 & $420-51871-474$ & J005300.53+150129.6 & $1.200 \pm 0.060$ & $8.136 \pm 0.042$ & 8.138 & $-1.282 \pm 0.012$ & $-0.748 \pm 0.008$ & $1.957 \pm 0.009$ & 0.633 \\
\hline 51 & 423- 51821- 402 & J011354.58+153947.8 & $1.265 \pm 0.036$ & $8.075 \pm 0.027$ & 8.131 & $-1.426 \pm 0.011$ & $-0.955 \pm 0.009$ & $2.214 \pm 0.008$ & 0.761 \\
\hline
\end{tabular}


S. Y. Yin et al.: Empirical strong-line oxygen abundance calibrations from galaxies with electron-temperature measurements, Online Material $p 3$

Table 1. continued.

\begin{tabular}{|c|c|c|c|c|c|c|c|c|c|}
\hline$(1)$ & $(2)$ & (3) & $\overline{(4)}$ & $\overline{(5)}$ & $\overline{(6)}$ & $\overline{(7)}$ & $\overline{(8)}$ & (9) & $(10)$ \\
\hline Num & Plate-MJD-FiberID & IAU designations & $t_{3}\left(10^{4} \mathrm{~K}\right)$ & $12+\log (\mathrm{O} / \mathrm{H})_{T_{\mathrm{e}}}$ & $(\mathrm{O} / \mathrm{H})_{\text {Bay }}$ & $\mathrm{N} 2$ & S 2 & $\mathrm{O} 3 \mathrm{~N} 2$ & $P$ \\
\hline 52 & $424-51893-368$ & $\mathrm{~J} 012223.90+152031.9$ & $1.146 \pm 0.036$ & $8.226 \pm 0.034$ & 8.812 & $-1.038 \pm 0.007$ & $-1.008 \pm 0.012$ & $1.897 \pm 0.005$ & 0.822 \\
\hline 53 & $430-51877-97$ & J015453.90+130721.7 & $1.173 \pm 0.083$ & $8.190 \pm 0.065$ & 8.165 & $-1.256 \pm 0.021$ & $-0.711 \pm 0.010$ & $1.971 \pm 0.016$ & 0.649 \\
\hline 54 & 431- 51877- 191 & J073256.71+370449.8 & $0.966 \pm 0.039$ & $8.372 \pm 0.042$ & 8.697 & $-0.981 \pm 0.005$ & $-0.851 \pm 0.007$ & $1.640 \pm 0.003$ & 0.684 \\
\hline 55 & 436- 51883- 391 & J075446.42+451502.7 & $0.975 \pm 0.045$ & $8.348 \pm 0.048$ & 8.615 & $-1.022 \pm 0.008$ & $-0.776 \pm 0.010$ & $1.677 \pm 0.006$ & 0.695 \\
\hline 56 & $442-51882-223$ & J082001.71+505039.1 & $1.099 \pm 0.046$ & $8.265 \pm 0.039$ & 8.499 & $-1.206 \pm 0.011$ & $-0.818 \pm 0.009$ & $1.941 \pm 0.010$ & 0.689 \\
\hline 57 & 443- 51873- 542 & J083108.23+493159.1 & $1.145 \pm 0.039$ & $8.177 \pm 0.032$ & 8.028 & $-1.407 \pm 0.012$ & $-0.896 \pm 0.008$ & $2.147 \pm 0.010$ & 0.732 \\
\hline 58 & 446- 51899- 86 & J084526.52+531916.2 & $1.011 \pm 0.050$ & $8.658 \pm 0.063$ & 8.196 & $-1.290 \pm 0.008$ & $-0.819 \pm 0.007$ & $2.010 \pm 0.006$ & 0.393 \\
\hline 59 & 446- 51899- 283 & J083516.99+533211.0 & $0.979 \pm 0.050$ & $8.409 \pm 0.051$ & 8.491 & $-1.202 \pm 0.007$ & $-0.796 \pm 0.006$ & $1.881 \pm 0.005$ & 0.642 \\
\hline 60 & 446- 51899- 352 & J083313.18+542222.8 & $1.062 \pm 0.054$ & $8.305 \pm 0.053$ & 8.403 & $-1.320 \pm 0.013$ & $-0.907 \pm 0.011$ & $2.106 \pm 0.011$ & 0.760 \\
\hline 61 & 446- 51899- 541 & J084703.00+545039.4 & $1.114 \pm 0.051$ & $8.231 \pm 0.042$ & 8.086 & $-1.404 \pm 0.023$ & $-0.780 \pm 0.008$ & $2.123 \pm 0.021$ & 0.684 \\
\hline 62 & 447- 51877- 172 & J084852.46+514532.7 & $1.058 \pm 0.065$ & $8.328 \pm 0.062$ & 8.112 & $-1.452 \pm 0.017$ & $-0.826 \pm 0.009$ & $2.212 \pm 0.014$ & 0.703 \\
\hline 63 & 452- 51911- 387 & J092635.26+582047.4 & $1.251 \pm 0.060$ & $8.123 \pm 0.048$ & 8.049 & $-1.750 \pm 0.065$ & $-1.119 \pm 0.037$ & $2.651 \pm 0.063$ & 0.870 \\
\hline 64 & 452- 51911- 487 & J093248.77+582530.7 & $1.018 \pm 0.039$ & $8.324 \pm 0.039$ & 8.500 & $-1.194 \pm 0.007$ & $-0.796 \pm 0.006$ & $1.883 \pm 0.005$ & 0.687 \\
\hline 65 & 454- 51908- 111 & J022907.37-085726.2 & $1.051 \pm 0.035$ & $8.318 \pm 0.034$ & 8.186 & $-1.328 \pm 0.011$ & $-0.894 \pm 0.011$ & $2.104 \pm 0.010$ & 0.747 \\
\hline 66 & 455- 51909- 369 & J023426.93-072807.6 & $1.187 \pm 0.077$ & $8.163 \pm 0.056$ & 8.149 & $-1.315 \pm 0.016$ & $-0.745 \pm 0.010$ & $2.015 \pm 0.014$ & 0.647 \\
\hline 67 & 456- 51910- 195 & J024453.66-082137.8 & $1.236 \pm 0.032$ & $8.101 \pm 0.023$ & 8.046 & $-1.493 \pm 0.010$ & $-0.926 \pm 0.008$ & $2.260 \pm 0.008$ & 0.740 \\
\hline 68 & 458- 51929- 42 & J030539.70-083905.4 & $1.261 \pm 0.075$ & $8.142 \pm 0.057$ & 8.207 & $-1.420 \pm 0.028$ & $-0.930 \pm 0.018$ & $2.312 \pm 0.026$ & 0.816 \\
\hline 69 & $458-51929-185$ & J030321.41-075923.2 & $1.480 \pm 0.024$ & $7.898 \pm 0.021$ & 7.898 & $-1.586 \pm 0.016$ & $-1.414 \pm 0.030$ & $2.482 \pm 0.014$ & 0.902 \\
\hline 70 & 459- 51924- 253 & J031023.95-083432.8 & $1.016 \pm 0.039$ & $8.377 \pm 0.042$ & 8.480 & $-1.334 \pm 0.010$ & $-0.958 \pm 0.007$ & $2.137 \pm 0.008$ & 0.767 \\
\hline 71 & $460-51924-128$ & J032101.01-080150.1 & $1.020 \pm 0.047$ & $8.333 \pm 0.049$ & 8.429 & $-1.263 \pm 0.008$ & $-0.843 \pm 0.007$ & $1.985 \pm 0.006$ & 0.714 \\
\hline 72 & $460-51924-592$ & J032613.63-063512.4 & $1.110 \pm 0.023$ & $8.269 \pm 0.023$ & 8.754 & $-1.101 \pm 0.007$ & $-0.937 \pm 0.009$ & $1.939 \pm 0.005$ & 0.798 \\
\hline 73 & 463- 51908- 299 & J034543.94-065446.0 & $1.126 \pm 0.064$ & $8.215 \pm 0.052$ & 8.362 & $-1.176 \pm 0.009$ & $-0.730 \pm 0.008$ & $1.853 \pm 0.007$ & 0.641 \\
\hline 74 & 467- 51901- 628 & J085201.82+010459.5 & $1.226 \pm 0.035$ & $8.104 \pm 0.025$ & 8.081 & $-1.387 \pm 0.012$ & $-0.858 \pm 0.008$ & $2.136 \pm 0.009$ & 0.725 \\
\hline 75 & $468-51912-250$ & $68-001117.8$ & $1.034 \pm 0.049$ & $8.344 \pm 0.046$ & 8.571 & $-1.212 \pm 0.016$ & $-0.846 \pm 0.012$ & $1.941 \pm 0.014$ & 0.688 \\
\hline 76 & 469- 51913- 110 & J090106.55+005418.0 & $1.192 \pm 0.024$ & $8.183 \pm 0.022$ & 8.307 & $-1.443 \pm 0.010$ & $-1.011 \pm 0.008$ & $2.306 \pm 0.008$ & 0.818 \\
\hline 77 & 472- 51955- 546 & J091652.23+003114.1 & $1.017 \pm 0.042$ & $8.344 \pm 0.043$ & 8.153 & $-1.342 \pm 0.009$ & $-0.882 \pm 0.007$ & $2.087 \pm 0.007$ & 0.733 \\
\hline 78 & 474- 52000- 610 & J092918.38+002813.0 & $1.298 \pm 0.023$ & $8.071 \pm 0.022$ & 8.041 & $-1.625 \pm 0.011$ & $-1.161 \pm 0.009$ & $2.532 \pm 0.009$ & 0.885 \\
\hline 79 & 483- 51924- 495 & J090047.45+574255.0 & $1.037 \pm 0.039$ & $8.322 \pm 0.041$ & 8.724 & $-1.059 \pm 0.006$ & $-0.910 \pm 0.009$ & $1.826 \pm 0.004$ & 0.757 \\
\hline 80 & 483- 51924- 594 & J090139.89+575945.9 & $1.087 \pm 0.040$ & $8.271 \pm 0.036$ & 8.124 & $-1.311 \pm 0.009$ & $-0.788 \pm 0.008$ & $2.047 \pm 0.006$ & 0.698 \\
\hline 81 & 489- 51930- 434 & $\mathrm{~J} 103007.42+654756.0$ & $1.171 \pm 0.079$ & $8.164 \pm 0.060$ & 8.103 & $-1.305 \pm 0.015$ & $-0.725 \pm 0.010$ & $2.005 \pm 0.012$ & 0.665 \\
\hline 82 & $490-51929-363$ & $\mathrm{~J} 105506.12+670918.3$ & $1.188 \pm 0.040$ & $8.150 \pm 0.028$ & 8.094 & $-1.338 \pm 0.008$ & $-0.818 \pm 0.006$ & $2.053 \pm 0.006$ & 0.678 \\
\hline 83 & $490-5$ & 54.1 & $1.211 \pm 0.038$ & $8.152 \pm 0.030$ & 8.100 & $-1.524 \pm 0.014$ & $-0.945 \pm 0.010$ & $2.353 \pm 0.012$ & 0.786 \\
\hline 84 & 493- 51957- 144 & $\mathrm{~J} 121333.58+665053.8$ & $1.280 \pm 0.066$ & $8.064 \pm 0.042$ & 8.129 & $-1.326 \pm 0.013$ & $-0.797 \pm 0.009$ & $2.022 \pm 0.009$ & 0.645 \\
\hline 85 & 493- 51957- 219 & $\mathrm{~J} 120821.93+661905.8$ & $1.217 \pm 0.054$ & $8.138 \pm 0.041$ & 8.097 & $-1.439 \pm 0.015$ & $-0.825 \pm 0.009$ & $2.222 \pm 0.013$ & 0.738 \\
\hline 86 & 502- 51957- 7 & $\mathrm{~J} 101430.99+004754.9$ & $1.185 \pm 0.024$ & $8.142 \pm 0.021$ & 8.481 & $-1.323 \pm 0.007$ & $-0.939 \pm 0.007$ & $2.112 \pm 0.005$ & 0.784 \\
\hline 87 & $505-52317-228$ & $\mathrm{~J} 103412.22+014248.9$ & $1.501 \pm 0.043$ & $7.858 \pm 0.026$ & 7.977 & $-1.757 \pm 0.023$ & $-1.104 \pm 0.012$ & $2.577 \pm 0.021$ & 0.826 \\
\hline 88 & $507-52353-87$ & $\mathrm{~J} 105331.42+011740.5$ & $1.231 \pm 0.038$ & $8.130 \pm 0.029$ & 8.112 & $-1.496 \pm 0.014$ & $-0.942 \pm 0.009$ & $2.305 \pm 0.012$ & 0.764 \\
\hline 89 & 507- 52353- 347 & $\mathrm{~J} 104642.48+022930.1$ & $1.126 \pm 0.060$ & $8.212 \pm 0.047$ & 8.472 & $-1.147 \pm 0.008$ & $-0.752 \pm 0.009$ & $1.804 \pm 0.006$ & 0.618 \\
\hline 90 & 509- 52374- 268 & J110719.03+015909.6 & $1.226 \pm 0.057$ & $8.199 \pm 0.051$ & 8.144 & $-1.742 \pm 0.043$ & $-1.286 \pm 0.040$ & $2.724 \pm 0.041$ & 0.909 \\
\hline 91 & 509- 52374- 592 & $\mathrm{~J} 111224.38+022734.5$ & $1.517 \pm 0.117$ & $7.903 \pm 0.054$ & 8.408 & $-1.049 \pm 0.014$ & $-0.636 \pm 0.013$ & $1.737 \pm 0.010$ & 0.595 \\
\hline 92 & 512- 51992- 524 & $\mathrm{~J} 112938.16+031503.9$ & $1.010 \pm 0.060$ & $8.359 \pm 0.057$ & 8.497 & $-1.104 \pm 0.008$ & $-0.713 \pm 0.006$ & $1.730 \pm 0.005$ & 0.585 \\
\hline 93 & 513- 51989- 521 & $\mathrm{~J} 113655.80+033333.4$ & $1.073 \pm 0.062$ & $8.257 \pm 0.053$ & 8.462 & $-1.145 \pm 0.010$ & $-0.739 \pm 0.010$ & $1.797 \pm 0.008$ & 0.638 \\
\hline 94 & 515- 52051- 378 & $\mathrm{~J} 115117.02+032656.0$ & $1.134 \pm 0.052$ & $8.237 \pm 0.042$ & 8.304 & $-1.236 \pm 0.012$ & $-0.835 \pm 0.010$ & $1.992 \pm 0.010$ & 0.696 \\
\hline 95 & 516- 52017- 403 & $\mathrm{~J} 120055.63+032403.9$ & $1.535 \pm 0.029$ & $7.841 \pm 0.022$ & 7.909 & $-1.806 \pm 0.019$ & $-1.257 \pm 0.016$ & $2.691 \pm 0.017$ & 0.906 \\
\hline 96 & 519- 52283- 507 & $\mathrm{~J} 122416.44+030036.0$ & $1.059 \pm 0.063$ & $8.286 \pm 0.058$ & 8.228 & $-1.238 \pm 0.011$ & $-0.773 \pm 0.012$ & $1.923 \pm 0.009$ & 0.665 \\
\hline 97 & 519- 52283- 615 & $\mathrm{~J} 122948.09+030658.6$ & $1.203 \pm 0.043$ & $8.112 \pm 0.033$ & 8.048 & $-1.359 \pm 0.012$ & $-0.804 \pm 0.009$ & $2.103 \pm 0.009$ & 0.739 \\
\hline 98 & 524- 52027- 16 & $\mathrm{~J} 130831.56+012208.0$ & $1.133 \pm 0.036$ & $8.228 \pm 0.032$ & 8.495 & $-1.304 \pm 0.008$ & $-0.928 \pm 0.008$ & $2.103 \pm 0.006$ & 0.761 \\
\hline 99 & 524- 52027- 260 & $\mathrm{~J} 130148.03+013718.4$ & $1.074 \pm 0.033$ & $8.319 \pm 0.033$ & 8.256 & $-1.412 \pm 0.010$ & $-0.949 \pm 0.009$ & $2.239 \pm 0.008$ & 0.774 \\
\hline 100 & 525- 52295- 626 & $\mathrm{~J} 131710.87+025620.0$ & $1.146 \pm 0.029$ & $8.218 \pm 0.027$ & 8.273 & $-1.421 \pm 0.023$ & $-0.992 \pm 0.010$ & $2.258 \pm 0.020$ & 0.804 \\
\hline 101 & 527- 52342- 247 & $\mathrm{~J} 132654.62+011346.9$ & $1.255 \pm 0.023$ & $8.108 \pm 0.020$ & 8.210 & $-1.415 \pm 0.010$ & $-0.999 \pm 0.011$ & $2.273 \pm 0.008$ & 0.823 \\
\hline 102 & $530-52026-525$ & $\mathrm{~J} 135155.89+032524.2$ & $1.052 \pm 0.036$ & $8.264 \pm 0.038$ & 8.834 & $-1.036 \pm 0.007$ & $-0.992 \pm 0.015$ & $1.787 \pm 0.005$ & 0.785 \\
\hline 103 & 535- 51999- 52 & $\mathrm{~J} 143210.15+012551.9$ & $1.086 \pm 0.038$ & $8.243 \pm 0.037$ & 8.823 & $-1.167 \pm 0.008$ & $-1.051 \pm 0.013$ & $1.939 \pm 0.006$ & 0.785 \\
\hline 104 & 539- 52017- 356 & $\mathrm{~J} 145814.74+020652.2$ & $1.111 \pm 0.050$ & $8.240 \pm 0.041$ & 8.239 & $-1.241 \pm 0.008$ & $-0.773 \pm 0.007$ & $1.950 \pm 0.007$ & 0.666 \\
\hline
\end{tabular}


Table 1. continued.

\begin{tabular}{|c|c|c|c|c|c|c|c|c|c|}
\hline$\overline{(1)}$ & $(2)$ & $\overline{(3)}$ & $\overline{(4)}$ & $\overline{(5)}$ & $\overline{(6)}$ & $\overline{(7)}$ & $\overline{(8)}$ & (9) & $(10)$ \\
\hline Num & Plate-MJD-FiberID & IAU designations & $t_{3}\left(10^{4} \mathrm{~K}\right)$ & $12+\log (\mathrm{O} / \mathrm{H})_{T_{\mathrm{e}}}$ & $(\mathrm{O} / \mathrm{H})_{\text {Bay }}$ & $\mathrm{N} 2$ & S2 & $\mathrm{O} 3 \mathrm{~N} 2$ & $P$ \\
\hline 105 & 544- 52201- 67 & J080139.46+382305.2 & $1.086 \pm 0.036$ & $8.258 \pm 0.036$ & 8.414 & $-1.353 \pm 0.010$ & $-0.947 \pm 0.008$ & $2.141 \pm 0.008$ & 0.781 \\
\hline 106 & 544- 52201- 610 & J080406.22+393656.8 & $1.067 \pm 0.068$ & $8.209 \pm 0.062$ & 8.624 & $-0.983 \pm 0.011$ & $-0.770 \pm 0.010$ & $1.610 \pm 0.009$ & 0.673 \\
\hline 107 & 548- 51986- 324 & J081829.71+453309.3 & $1.339 \pm 0.098$ & $8.011 \pm 0.054$ & 8.163 & $-1.227 \pm 0.012$ & $-0.676 \pm 0.008$ & $1.855 \pm 0.010$ & 0.572 \\
\hline 108 & 548- 51986- 347 & J082059.30+461823.4 & $1.027 \pm 0.043$ & $8.314 \pm 0.043$ & 8.052 & $-1.310 \pm 0.008$ & $-0.810 \pm 0.006$ & $2.026 \pm 0.006$ & 0.715 \\
\hline 109 & 549- 51981- 90 & J083350.23+454933.6 & $0.947 \pm 0.049$ & $8.453 \pm 0.056$ & 8.591 & $-1.137 \pm 0.012$ & $-0.832 \pm 0.009$ & $1.855 \pm 0.011$ & 0.697 \\
\hline 110 & $550-51959-485$ & J083914.95+481518.3 & $1.188 \pm 0.081$ & $8.130 \pm 0.058$ & 8.248 & $-1.175 \pm 0.011$ & $-0.712 \pm 0.008$ & $1.801 \pm 0.008$ & 0.598 \\
\hline 111 & 553- 51999- 134 & J091052.58+522756.5 & $1.040 \pm 0.043$ & $8.335 \pm 0.039$ & 8.282 & $-1.234 \pm 0.007$ & $-0.777 \pm 0.005$ & $1.915 \pm 0.004$ & 0.628 \\
\hline 112 & 555- 52266- 517 & J093126.23+551221.6 & $1.121 \pm 0.030$ & $8.244 \pm 0.025$ & 8.547 & $-1.270 \pm 0.008$ & $-0.892 \pm 0.007$ & $2.045 \pm 0.006$ & 0.731 \\
\hline 113 & 557- 52253- 590 & J095513.13+573203.1 & $1.169 \pm 0.074$ & $8.152 \pm 0.056$ & 8.603 & $-1.148 \pm 0.011$ & $-0.798 \pm 0.012$ & $1.842 \pm 0.009$ & 0.683 \\
\hline 114 & 560- 52296- 204 & $\mathrm{~J} 103103.99+585602.0$ & $1.075 \pm 0.066$ & $8.199 \pm 0.054$ & 8.699 & $-0.847 \pm 0.006$ & $-0.727 \pm 0.008$ & $1.381 \pm 0.004$ & 0.565 \\
\hline 115 & 564- 52224- 363 & J084216.95+033806.7 & $1.094 \pm 0.044$ & $8.298 \pm 0.043$ & 8.272 & $-1.315 \pm 0.046$ & $-1.000 \pm 0.018$ & $2.178 \pm 0.044$ & 0.819 \\
\hline 116 & 566- 52238- 497 & J090531.08+033530.4 & $1.484 \pm 0.018$ & $7.871 \pm 0.018$ & 7.914 & $-1.758 \pm 0.012$ & $-1.207 \pm 0.008$ & $2.632 \pm 0.009$ & 0.898 \\
\hline 117 & 568- 52254- 244 & J092251.26+025607.4 & $1.078 \pm 0.049$ & $8.277 \pm 0.041$ & 8.575 & $-1.078 \pm 0.007$ & $-0.747 \pm 0.006$ & $1.765 \pm 0.005$ & 0.648 \\
\hline 118 & 569- 52264- 609 & J093815.91+043408.4 & $1.106 \pm 0.037$ & $8.245 \pm 0.035$ & 8.713 & $-1.243 \pm 0.009$ & $-0.981 \pm 0.008$ & $2.054 \pm 0.007$ & 0.798 \\
\hline 119 & $570-52266-61$ & J094646.66+033812.8 & $1.145 \pm 0.061$ & $8.220 \pm 0.053$ & 8.557 & $-1.196 \pm 0.011$ & $-0.928 \pm 0.015$ & $2.016 \pm 0.008$ & 0.779 \\
\hline 120 & 582- 52045- 445 & $\mathrm{~J} 140725.32+052837.9$ & $1.234 \pm 0.028$ & $8.103 \pm 0.025$ & 8.032 & $-1.533 \pm 0.012$ & $-1.059 \pm 0.013$ & $2.373 \pm 0.010$ & 0.834 \\
\hline 121 & 587- 52026- 155 & J144610.32+033921.6 & $1.105 \pm 0.039$ & $8.228 \pm 0.030$ & 8.573 & $-1.099 \pm 0.006$ & $-0.741 \pm 0.007$ & $1.773 \pm 0.004$ & 0.648 \\
\hline 122 & 587- 52026- 495 & $\mathrm{~J} 144441.37+040941.7$ & $1.679 \pm 0.022$ & $7.841 \pm 0.021$ & 7.930 & $-2.036 \pm 0.029$ & $-1.621 \pm 0.025$ & $3.052 \pm 0.027$ & 0.961 \\
\hline 123 & 588- 52045- 483 & $\mathrm{~J} 145424.60+035925.0$ & $1.183 \pm 0.051$ & $8.177 \pm 0.036$ & 8.573 & $-1.069 \pm 0.009$ & $-0.803 \pm 0.010$ & $1.799 \pm 0.007$ & 0.671 \\
\hline 124 & 589- 52055- 483 & $\mathrm{~J} 150339.41+035051.7$ & $1.159 \pm 0.078$ & $8.138 \pm 0.062$ & 8.574 & $-1.051 \pm 0.010$ & $-0.764 \pm 0.009$ & $1.728 \pm 0.007$ & 0.682 \\
\hline 125 & $590-52057-200$ & $\mathrm{~J} 151045.41+033038.5$ & $1.094 \pm 0.057$ & $8.272 \pm 0.049$ & 8.213 & $-1.231 \pm 0.010$ & $-0.748 \pm 0.007$ & $1.925 \pm 0.006$ & 0.635 \\
\hline 126 & 594- 52045- 493 & $\mathrm{~J} 154654.55+030902.1$ & $1.154 \pm 0.025$ & $8.209 \pm 0.022$ & 8.554 & $-1.327 \pm 0.009$ & $-0.971 \pm 0.007$ & $2.150 \pm 0.007$ & 0.788 \\
\hline 127 & $597-52059-460$ & $\mathrm{~J} 113303.79+651341.1$ & $1.363 \pm 0.053$ & $8.012 \pm 0.035$ & 8.193 & $-1.420 \pm 0.032$ & $-1.098 \pm 0.041$ & $2.292 \pm 0.030$ & 0.842 \\
\hline 128 & 597- 52059- 586 & $\mathrm{~J} 114047.42+644710.3$ & $1.102 \pm 0.032$ & $8.274 \pm 0.029$ & 8.313 & $-1.345 \pm 0.008$ & $-0.920 \pm 0.007$ & $2.133 \pm 0.006$ & 0.735 \\
\hline 129 & $602-52072-19$ & $\mathrm{~J} 131426.57+633311.5$ & $1.057 \pm 0.052$ & $8.302 \pm 0.048$ & 8.595 & $-1.142 \pm 0.007$ & $-0.833 \pm 0.007$ & $1.854 \pm 0.005$ & 0.681 \\
\hline 130 & $605-52353-616$ & $\mathrm{~J} 141145.34+623911.1$ & $1.106 \pm 0.052$ & $8.269 \pm 0.048$ & 8.508 & $-1.258 \pm 0.016$ & $-0.921 \pm 0.029$ & $2.081 \pm 0.014$ & 0.786 \\
\hline 131 & 606- 52365- 604 & $\mathrm{~J} 142619.15+622750.0$ & $1.048 \pm 0.039$ & $8.328 \pm 0.039$ & 8.559 & $-1.329 \pm 0.010$ & $-0.972 \pm 0.008$ & $2.128 \pm 0.008$ & 0.771 \\
\hline 132 & 608- 52081- 255 & $\mathrm{~J} 144329.11+585543.6$ & $1.195 \pm 0.084$ & $8.116 \pm 0.064$ & 8.043 & $-1.375 \pm 0.014$ & $-0.816 \pm 0.010$ & $2.079 \pm 0.011$ & 0.695 \\
\hline 133 & 609- 52339- 441 & $\mathrm{~J} 144845.84+634620.2$ & $1.062 \pm 0.054$ & $8.323 \pm 0.053$ & 8.203 & $-1.346 \pm 0.012$ & $-0.874 \pm 0.019$ & $2.145 \pm 0.010$ & 0.752 \\
\hline 134 & 613- 52345- 342 & $\mathrm{~J} 150728.68+595913.2$ & $1.138 \pm 0.034$ & $8.164 \pm 0.028$ & 8.759 & $-1.039 \pm 0.006$ & $-0.909 \pm 0.008$ & $1.773 \pm 0.004$ & 0.753 \\
\hline 135 & 615- 52347- 606 & $\mathrm{~J} 153737.27+584740.5$ & $1.253 \pm 0.053$ & $8.124 \pm 0.041$ & 8.542 & $-1.147 \pm 0.046$ & $-1.027 \pm 0.023$ & $2.023 \pm 0.045$ & 0.833 \\
\hline 136 & 616- 52374- 349 & $\mathrm{~J} 153534.13+545534.3$ & $1.102 \pm 0.037$ & $8.269 \pm 0.032$ & 8.613 & $-1.132 \pm 0.007$ & $-0.891 \pm 0.011$ & $1.906 \pm 0.005$ & 0.727 \\
\hline 137 & $620-5$ & $.49+520650.7$ & $1.032 \pm 0.061$ & $8.285 \pm 0.057$ & 8.621 & $-1.031 \pm 0.007$ & $-0.778 \pm 0.008$ & $1.659 \pm 0.005$ & 0.637 \\
\hline 138 & $620-52375-506$ & $\mathrm{~J} 160041.62+514443.0$ & $1.230 \pm 0.060$ & $8.132 \pm 0.047$ & 8.118 & $-1.319 \pm 0.017$ & $-0.742 \pm 0.009$ & $2.075 \pm 0.008$ & 0.696 \\
\hline 139 & 621- 52055- 618 & $\mathrm{~J} 161156.30+532630.1$ & $1.064 \pm 0.047$ & $8.305 \pm 0.041$ & 8.144 & $-1.275 \pm 0.010$ & $-0.745 \pm 0.007$ & $1.970 \pm 0.008$ & 0.642 \\
\hline 140 & 623- 52051- 396 & $\mathrm{~J} 161031.34+522305.9$ & $1.405 \pm 0.057$ & $7.905 \pm 0.037$ & 7.929 & $-1.628 \pm 0.025$ & $-1.058 \pm 0.016$ & $2.426 \pm 0.023$ & 0.833 \\
\hline 141 & $625-52145-72$ & $\mathrm{~J} 163106.19+484527.0$ & $1.812 \pm 0.177$ & $7.664 \pm 0.058$ & 8.148 & $-1.216 \pm 0.017$ & $-0.632 \pm 0.012$ & $1.810 \pm 0.013$ & 0.550 \\
\hline 142 & $627-52144-253$ & $\mathrm{~J} 163240.72+463338.1$ & $0.986 \pm 0.054$ & $8.365 \pm 0.056$ & 8.681 & $-0.977 \pm 0.006$ & $-0.823 \pm 0.009$ & $1.665 \pm 0.005$ & 0.697 \\
\hline 143 & 627- 52144- 295 & $\mathrm{~J} 163055.78+461805.7$ & $1.052 \pm 0.050$ & $8.329 \pm 0.050$ & 8.566 & $-1.184 \pm 0.010$ & $-0.895 \pm 0.014$ & $1.976 \pm 0.009$ & 0.753 \\
\hline 144 & 627- 52144- 446 & $\mathrm{~J} 163445.34+465903.1$ & $1.010 \pm 0.040$ & $8.372 \pm 0.043$ & 8.534 & $-1.212 \pm 0.011$ & $-0.853 \pm 0.009$ & $1.980 \pm 0.009$ & 0.741 \\
\hline 145 & $628-52083-555$ & $\mathrm{~J} 164235.52+422349.5$ & $1.105 \pm 0.031$ & $8.232 \pm 0.026$ & 8.589 & $-1.164 \pm 0.006$ & $-0.857 \pm 0.007$ & $1.893 \pm 0.004$ & 0.707 \\
\hline 146 & 629-52051- 497 & $\mathrm{~J} 164359.16+443632.7$ & $0.957 \pm 0.045$ & $8.473 \pm 0.051$ & 8.481 & $-1.260 \pm 0.008$ & $-0.872 \pm 0.007$ & $2.002 \pm 0.006$ & 0.678 \\
\hline 147 & 631- 52079- 307 & $\mathrm{~J} 164645.10+413208.8$ & $1.242 \pm 0.091$ & $8.064 \pm 0.066$ & 8.023 & $-1.430 \pm 0.019$ & $-0.826 \pm 0.013$ & $2.154 \pm 0.016$ & 0.725 \\
\hline 148 & 633- 52079- 336 & $\mathrm{~J} 165711.23+401915.6$ & $1.080 \pm 0.064$ & $8.273 \pm 0.061$ & 8.291 & $-1.249 \pm 0.011$ & $-0.829 \pm 0.009$ & $2.032 \pm 0.009$ & 0.765 \\
\hline 149 & 636- 52176- 439 & J205430.86-061739.8 & $1.217 \pm 0.089$ & $8.116 \pm 0.062$ & 8.300 & $-1.142 \pm 0.012$ & $-0.706 \pm 0.008$ & $1.831 \pm 0.010$ & 0.650 \\
\hline 150 & 637- 52174- 399 & $\mathrm{~J} 210114.40-0$ & $1.056 \pm 0.029$ & $8.360 \pm 0.029$ & 8.401 & $-1.333 \pm 0.011$ & $-0.905 \pm 0.008$ & $2.165 \pm 0.010$ & 0.759 \\
\hline 151 & 639- 52146- 143 & $\mathrm{~J} 211902.28-074226.6$ & $1.276 \pm 0.047$ & $8.048 \pm 0.033$ & 8.032 & $-1.447 \pm 0.011$ & $-0.907 \pm 0.010$ & $2.210 \pm 0.009$ & 0.753 \\
\hline 152 & $648-52559-288$ & J234148.29-105619.6 & $1.049 \pm 0.048$ & $8.279 \pm 0.045$ & 8.163 & $-1.223 \pm 0.008$ & $-0.754 \pm 0.006$ & $1.905 \pm 0.006$ & 0.681 \\
\hline 153 & 649- 52201- 637 & J235604.68-085423.4 & $1.121 \pm 0.044$ & $8.233 \pm 0.040$ & 8.120 & $-1.427 \pm 0.016$ & $-0.933 \pm 0.014$ & $2.227 \pm 0.014$ & 0.778 \\
\hline 154 & 651- 52141- 304 & J000430.34-101129.7 & $1.489 \pm 0.055$ & $7.865 \pm 0.033$ & 7.933 & $-1.594 \pm 0.034$ & $-1.322 \pm 0.047$ & $2.434 \pm 0.033$ & 0.856 \\
\hline 155 & 656- 52148- 140 & J004645.72-105410.4 & $1.128 \pm 0.072$ & $8.283 \pm 0.063$ & 8.290 & $-1.346 \pm 0.015$ & $-0.886 \pm 0.011$ & $2.196 \pm 0.012$ & 0.765 \\
\hline 156 & 658- 52146- 17 & J010513.49-103740.8 & $1.187 \pm 0.041$ & $8.202 \pm 0.035$ & 8.136 & $-1.529 \pm 0.014$ & $-0.978 \pm 0.009$ & $2.377 \pm 0.012$ & 0.780 \\
\hline 157 & 659- 52199- 307 & J010409.91-095346.6 & $1.205 \pm 0.074$ & $8.129 \pm 0.055$ & 8.060 & $-1.486 \pm 0.019$ & $-0.830 \pm 0.011$ & $2.218 \pm 0.017$ & 0.701 \\
\hline
\end{tabular}


Table 1. continued.

\begin{tabular}{|c|c|c|c|c|c|c|c|c|c|}
\hline (1) & $(2)$ & (3) & (4) & (5) & (6) & (7) & (8) & (9) & (10) \\
\hline Num & Plate-MJD-FiberID & IAU designations & $t_{3}\left(10^{4} \mathrm{~K}\right)$ & $12+\log (\mathrm{O} / \mathrm{H})_{T_{\mathrm{e}}}$ & $(\mathrm{O} / \mathrm{H})_{\text {Bay }}$ & N2 & S2 & O3N2 & $P$ \\
\hline 158 & $659-52199-435$ & J010643.32-092223.1 & $1.117 \pm 0.059$ & $8.255 \pm 0.054$ & 8.159 & $-1.369 \pm 0.016$ & $-0.871 \pm 0.012$ & $2.171 \pm 0.012$ & 0.755 \\
\hline 159 & $660-52177-414$ & J011616.82-085021.4 & $1.263 \pm 0.041$ & $8.060 \pm 0.029$ & 8.070 & $-1.386 \pm 0.011$ & $-0.900 \pm 0.008$ & $2.154 \pm 0.009$ & 0.756 \\
\hline 160 & $660-52177-450$ & J011729.09-084403.8 & $1.045 \pm 0.038$ & $8.305 \pm 0.036$ & 8.567 & $-1.139 \pm 0.008$ & $-0.824 \pm 0.009$ & $1.855 \pm 0.005$ & 0.697 \\
\hline 161 & 663- 52145- 406 & J013844.90-083540.5 & $1.233 \pm 0.046$ & $8.118 \pm 0.033$ & 8.114 & $-1.404 \pm 0.013$ & $-0.860 \pm 0.009$ & $2.184 \pm 0.011$ & 0.738 \\
\hline 162 & 664- 52174- 112 & J014721.67-091646.2 & $1.139 \pm 0.042$ & $8.218 \pm 0.036$ & 8.123 & $-1.391 \pm 0.016$ & $-0.841 \pm 0.013$ & $2.177 \pm 0.014$ & 0.750 \\
\hline 163 & 664- 52174- 447 & J014713.82-080702.6 & $1.251 \pm 0.063$ & $8.056 \pm 0.043$ & 8.038 & $-1.388 \pm 0.014$ & $-0.797 \pm 0.009$ & $2.079 \pm 0.011$ & 0.682 \\
\hline 164 & 666- 52149- 532 & J020356.90-080758.4 & $1.027 \pm 0.048$ & $8.307 \pm 0.043$ & 8.594 & $-1.091 \pm 0.007$ & $-0.759 \pm 0.007$ & $1.748 \pm 0.006$ & 0.653 \\
\hline 165 & $668-52162-89$ & J022037.66-092907.4 & $1.220 \pm 0.028$ & $8.140 \pm 0.023$ & 8.407 & $-1.144 \pm 0.006$ & $-0.985 \pm 0.007$ & $1.973 \pm 0.005$ & 0.793 \\
\hline 166 & 716- 52203- 368 & $\mathrm{~J} 215637.75-070007.9$ & $1.198 \pm 0.082$ & $8.110 \pm 0.058$ & 8.352 & $-1.138 \pm 0.009$ & $-0.710 \pm 0.008$ & $1.732 \pm 0.007$ & 0.574 \\
\hline 167 & 726- 52226- 532 & J231322.68-084503.9 & $1.080 \pm 0.068$ & $8.274 \pm 0.056$ & 8.484 & $-1.081 \pm 0.008$ & $-0.686 \pm 0.007$ & $1.706 \pm 0.006$ & 0.576 \\
\hline 168 & 730- 52466- 376 & $\mathrm{~J} 212442.05+114751.0$ & $1.014 \pm 0.037$ & $8.324 \pm 0.036$ & 8.610 & $-1.007 \pm 0.005$ & $-0.816 \pm 0.005$ & $1.687 \pm 0.003$ & 0.682 \\
\hline 169 & 735- 52519- 461 & J220802.88+131334.6 & $1.192 \pm 0.041$ & $8.127 \pm 0.033$ & 8.485 & $-1.295 \pm 0.009$ & $-0.908 \pm 0.008$ & $2.064 \pm 0.007$ & 0.768 \\
\hline 170 & $740-52263-267$ & $\mathrm{~J} 224422.71+131608.7$ & $1.021 \pm 0.063$ & $8.338 \pm 0.062$ & 8.544 & $-1.150 \pm 0.010$ & $-0.776 \pm 0.007$ & $1.846 \pm 0.008$ & 0.673 \\
\hline 171 & 753- 52233- 593 & $\mathrm{~J} 002652.08+152737.8$ & $1.273 \pm 0.066$ & $8.048 \pm 0.049$ & 8.722 & $-1.249 \pm 0.031$ & $-1.026 \pm 0.027$ & $2.081 \pm 0.028$ & 0.845 \\
\hline 172 & $755-52235-300$ & J073928.39+314502.1 & $1.086 \pm 0.039$ & $8.264 \pm 0.037$ & 8.063 & $-1.367 \pm 0.009$ & $-0.810 \pm 0.006$ & $2.130 \pm 0.007$ & 0.743 \\
\hline 173 & 756- 52577- 37 & J075507.92+340827.6 & $1.146 \pm 0.035$ & $8.185 \pm 0.030$ & 8.729 & $-1.012 \pm 0.006$ & $-0.876 \pm 0.009$ & $1.778 \pm 0.005$ & 0.758 \\
\hline 174 & 761- 52266- 443 & J082542.86+422758.6 & $1.342 \pm 0.025$ & $8.032 \pm 0.018$ & 8.117 & $-1.506 \pm 0.011$ & $-0.945 \pm 0.012$ & $2.339 \pm 0.009$ & 0.788 \\
\hline 175 & 761- 52266- 454 & J082614.16+422833.6 & $1.123 \pm 0.074$ & $8.241 \pm 0.057$ & 8.431 & $-1.097 \pm 0.009$ & $-0.694 \pm 0.007$ & $1.725 \pm 0.007$ & 0.560 \\
\hline 176 & $762-52232-217$ & J083343.87+431951.6 & $1.002 \pm 0.058$ & $8.363 \pm 0.056$ & 8.157 & $-1.207 \pm 0.008$ & $-0.705 \pm 0.007$ & $1.852 \pm 0.006$ & 0.614 \\
\hline 177 & $762-52232-575$ & $\mathrm{~J} 083803.72+445900.2$ & $1.205 \pm 0.050$ & $8.099 \pm 0.036$ & 8.598 & $-1.186 \pm 0.011$ & $-0.821 \pm 0.010$ & $1.876 \pm 0.009$ & 0.689 \\
\hline 178 & $767-52252-463$ & J092429.86+514301.2 & $1.208 \pm 0.048$ & $8.117 \pm 0.032$ & 8.124 & $-1.284 \pm 0.009$ & $-0.740 \pm 0.006$ & $1.960 \pm 0.007$ & 0.644 \\
\hline 179 & 768-52281- 193 & J093733.77+523924.8 & $1.114 \pm 0.068$ & $8.237 \pm 0.054$ & 8.290 & $-1.197 \pm 0.009$ & $-0.743 \pm 0.007$ & $1.851 \pm 0.007$ & 0.603 \\
\hline 180 & 769- 52282- 100 & J095131.78+525936.2 & $1.268 \pm 0.021$ & $8.087 \pm 0.021$ & 8.027 & $-1.599 \pm 0.009$ & $-1.103 \pm 0.006$ & $2.468 \pm 0.007$ & 0.849 \\
\hline 181 & 769- 52282- 575 & J095408.42+544606.9 & $1.069 \pm 0.040$ & $8.318 \pm 0.037$ & 8.366 & $-1.247 \pm 0.034$ & $-0.874 \pm 0.009$ & $2.023 \pm 0.032$ & 0.719 \\
\hline 182 & $771-52370-490$ & $\mathrm{~J} 101242.98+613302.8$ & $1.123 \pm 0.030$ & $8.248 \pm 0.028$ & 8.105 & $-1.453 \pm 0.009$ & $-0.914 \pm 0.007$ & $2.257 \pm 0.007$ & 0.757 \\
\hline 183 & $772-52375-270$ & $\mathrm{~J} 102132.50+614404.5$ & $1.277 \pm 0.067$ & $8.086 \pm 0.045$ & 8.303 & $-1.263 \pm 0.014$ & $-0.872 \pm 0.012$ & $2.031 \pm 0.011$ & 0.709 \\
\hline 184 & 773- 52376- 143 & $\mathrm{~J} 104500.96+621527.3$ & $1.269 \pm 0.083$ & $8.058 \pm 0.048$ & 8.594 & $-0.691 \pm 0.005$ & $-0.477 \pm 0.006$ & $1.218 \pm 0.003$ & 0.491 \\
\hline 185 & 775- 52295- 291 & $\mathrm{~J} 111439.19+605418.3$ & $1.163 \pm 0.039$ & $8.199 \pm 0.030$ & 8.311 & $-1.284 \pm 0.009$ & $-0.850 \pm 0.007$ & $2.034 \pm 0.007$ & 0.693 \\
\hline 186 & 779- 52342- 355 & $\mathrm{~J} 120725.66+623458.0$ & $0.993 \pm 0.043$ & $8.310 \pm 0.043$ & 8.709 & $-0.970 \pm 0.006$ & $-0.830 \pm 0.006$ & $1.579 \pm 0.003$ & 0.652 \\
\hline 187 & $782-52320-22$ & $\mathrm{~J} 130445.62+622420.8$ & $0.961 \pm 0.042$ & $8.382 \pm 0.045$ & 8.695 & $-0.936 \pm 0.005$ & $-0.813 \pm 0.005$ & $1.580 \pm 0.003$ & 0.663 \\
\hline 188 & 784- 52327- 179 & $\mathrm{~J} 132002.90+614627.4$ & $1.247 \pm 0.088$ & $8.109 \pm 0.059$ & 8.196 & $-1.247 \pm 0.015$ & $-0.769 \pm 0.011$ & $1.937 \pm 0.012$ & 0.624 \\
\hline 189 & 792-52353- 110 & $\mathrm{~J} 150127.22+553126.3$ & $1.113 \pm 0.072$ & $8.255 \pm 0.059$ & 8.160 & $-1.284 \pm 0.012$ & $-0.741 \pm 0.009$ & $1.952 \pm 0.009$ & 0.601 \\
\hline 190 & 794- 52376- 460 & $\mathrm{~J} 152045.65+533807.0$ & $1.070 \pm 0.054$ & $8.326 \pm 0.054$ & 8.421 & $-1.246 \pm 0.019$ & $-0.887 \pm 0.015$ & $2.072 \pm 0.015$ & 0.770 \\
\hline 191 & 817- 52381- 424 & $\mathrm{~J} 163210.01+412055.6$ & $1.105 \pm 0.043$ & $8.226 \pm 0.036$ & 8.487 & $-1.164 \pm 0.009$ & $-0.771 \pm 0.009$ & $1.856 \pm 0.006$ & 0.672 \\
\hline 192 & 828- 52317- 319 & J083145.67+384756.7 & $1.107 \pm 0.067$ & $8.257 \pm 0.059$ & 8.188 & $-1.310 \pm 0.014$ & $-0.846 \pm 0.013$ & $2.074 \pm 0.011$ & 0.718 \\
\hline 193 & 834- 52316- 157 & $\mathrm{~J} 093855.90+471228.8$ & $1.100 \pm 0.053$ & $8.259 \pm 0.047$ & 8.096 & $-1.384 \pm 0.011$ & $-0.810 \pm 0.008$ & $2.121 \pm 0.009$ & 0.695 \\
\hline 194 & 836- 52376- 378 & $\mathrm{~J} 112145.51+055756.5$ & $1.066 \pm 0.039$ & $8.300 \pm 0.042$ & 8.537 & $-1.298 \pm 0.010$ & $-0.934 \pm 0.011$ & $2.129 \pm 0.008$ & 0.821 \\
\hline 195 & $838-52378-595$ & $\mathrm{~J} 114359.54+052154.7$ & $1.459 \pm 0.033$ & $7.881 \pm 0.024$ & 8.717 & $-1.457 \pm 0.014$ & $-1.172 \pm 0.013$ & $2.288 \pm 0.011$ & 0.854 \\
\hline 196 & $841-52375-333$ & $\mathrm{~J} 115641.37+054315.6$ & $1.082 \pm 0.067$ & $8.251 \pm 0.059$ & 8.567 & $-1.120 \pm 0.009$ & $-0.755 \pm 0.008$ & $1.793 \pm 0.007$ & 0.655 \\
\hline 197 & 845- 52381- 1 & $\mathrm{~J} 123523.86+034536.0$ & $1.099 \pm 0.049$ & $8.255 \pm 0.044$ & 8.159 & $-1.332 \pm 0.011$ & $-0.828 \pm 0.018$ & $2.094 \pm 0.008$ & 0.732 \\
\hline 198 & 846- 52407- 267 & $\mathrm{~J} 123538.74+041245.0$ & $1.113 \pm 0.041$ & $8.260 \pm 0.034$ & 8.420 & $-1.246 \pm 0.007$ & $-0.826 \pm 0.006$ & $1.997 \pm 0.005$ & 0.691 \\
\hline 199 & $847-52426-522$ & J124954.84+060610.4 & $1.105 \pm 0.035$ & $8.230 \pm 0.029$ & 8.338 & $-1.233 \pm 0.007$ & $-0.799 \pm 0.006$ & $1.932 \pm 0.005$ & 0.676 \\
\hline 200 & 848- 52669- 528 & $\mathrm{~J} 125657.58+055616.4$ & $0.949 \pm 0.055$ & $8.368 \pm 0.059$ & 8.678 & $-0.972 \pm 0.006$ & $-0.790 \pm 0.007$ & $1.542 \pm 0.003$ & 0.611 \\
\hline 201 & 853- 52374- 577 & $\mathrm{~J} 133810.13+053504.5$ & $1.000 \pm 0.055$ & $8.332 \pm 0.054$ & 8.672 & $-1.030 \pm 0.006$ & $-0.819 \pm 0.006$ & $1.640 \pm 0.004$ & 0.616 \\
\hline 202 & 857- 52314- 386 & J074034.66+244136.6 & $1.096 \pm 0.038$ & $8.277 \pm 0.035$ & 8.366 & $-1.312 \pm 0.029$ & $-0.944 \pm 0.012$ & $2.117 \pm 0.027$ & 0.763 \\
\hline 203 & $859-52317-70$ & $\mathrm{~J} 080000.70+274641.8$ & $1.117 \pm 0.031$ & $8.269 \pm 0.030$ & 8.189 & $-1.408 \pm 0.009$ & $-0.939 \pm 0.007$ & $2.245 \pm 0.006$ & 0.781 \\
\hline 204 & $864-52320-379$ & $\mathrm{~J} 083833.53+374216.5$ & $1.137 \pm 0.064$ & $8.239 \pm 0.059$ & 8.416 & $-1.415 \pm 0.034$ & $-1.141 \pm 0.039$ & $2.306 \pm 0.032$ & 0.862 \\
\hline 205 & $870-52325-321$ & J092600.41+442736.0 & $1.268 \pm 0.029$ & $8.094 \pm 0.023$ & 8.459 & $-1.409 \pm 0.011$ & $-1.020 \pm 0.011$ & $2.257 \pm 0.009$ & 0.813 \\
\hline 206 & $879-52365-14$ & $\mathrm{~J} 113247.69+510215.3$ & $1.260 \pm 0.046$ & $8.087 \pm 0.034$ & 8.724 & $-1.305 \pm 0.016$ & $-1.047 \pm 0.010$ & $2.116 \pm 0.014$ & 0.785 \\
\hline 207 & 881- 52368- 567 & $\mathrm{~J} 115240.85+533228.3$ & $0.997 \pm 0.049$ & $8.303 \pm 0.051$ & 8.603 & $-0.986 \pm 0.006$ & $-0.737 \pm 0.006$ & $1.627 \pm 0.004$ & 0.692 \\
\hline 208 & 884- 52374- 404 & $\mathrm{~J} 122611.16+532602.0$ & $1.497 \pm 0.027$ & $7.853 \pm 0.022$ & 7.937 & $-1.858 \pm 0.019$ & $-1.138 \pm 0.009$ & $2.696 \pm 0.015$ & 0.861 \\
\hline 209 & 886- 52381- 129 & $\mathrm{~J} 125427.22+510436.1$ & $1.197 \pm 0.083$ & $8.153 \pm 0.061$ & 8.184 & $-1.267 \pm 0.013$ & $-0.779 \pm 0.010$ & $1.982 \pm 0.010$ & 0.661 \\
\hline 210 & $886-52381-605$ & $\mathrm{~J} 125931.51+531554.0$ & $1.044 \pm 0.049$ & $8.305 \pm 0.046$ & 8.601 & $-1.092 \pm 0.008$ & $-0.815 \pm 0.008$ & $1.787 \pm 0.006$ & 0.678 \\
\hline
\end{tabular}


Table 1. continued.

\begin{tabular}{|c|c|c|c|c|c|c|c|c|c|}
\hline (1) & (2) & (3) & (4) & (5) & (6) & (7) & (8) & (9) & (10) \\
\hline Num & Plate-MJD-FiberID & IAU designations & $t_{3}\left(10^{4} \mathrm{~K}\right)$ & $12+\log (\mathrm{O} / \mathrm{H})_{T_{\mathrm{e}}}$ & $(\mathrm{O} / \mathrm{H})_{\text {Bay }}$ & $\mathrm{N} 2$ & S2 & O3N2 & $P$ \\
\hline 211 & $890-52583-49$ & J075308.57+304512.6 & $1.039 \pm 0.044$ & $8.356 \pm 0.045$ & 8.227 & $-1.299 \pm 0.010$ & $-0.839 \pm 0.009$ & $2.093 \pm 0.008$ & 0.742 \\
\hline 212 & $890-52583-65$ & $\mathrm{~J} 075454.69+312821.0$ & $1.057 \pm 0.063$ & $8.332 \pm 0.063$ & 8.336 & $-1.352 \pm 0.016$ & $-0.943 \pm 0.011$ & $2.178 \pm 0.014$ & 0.783 \\
\hline 213 & $893-52589-76$ & $\mathrm{~J} 082010.56+374354.4$ & $1.366 \pm 0.081$ & $8.022 \pm 0.046$ & 8.171 & $-1.315 \pm 0.016$ & $-0.764 \pm 0.010$ & $2.055 \pm 0.014$ & 0.657 \\
\hline 214 & 893- 52589- 177 & J081755.35+372959.6 & $1.137 \pm 0.066$ & $8.170 \pm 0.048$ & 8.501 & $-1.073 \pm 0.007$ & $-0.676 \pm 0.006$ & $1.626 \pm 0.004$ & 0.541 \\
\hline 215 & $896-52592-125$ & $\mathrm{~J} 084419.08+414310.2$ & $1.063 \pm 0.039$ & $8.256 \pm 0.035$ & 8.714 & $-0.925 \pm 0.005$ & $-0.832 \pm 0.007$ & $1.614 \pm 0.003$ & 0.696 \\
\hline 216 & 901- 52641- 536 & J093816.85+504200.0 & $1.253 \pm 0.038$ & $8.101 \pm 0.027$ & 8.260 & $-1.341 \pm 0.015$ & $-0.903 \pm 0.013$ & $2.124 \pm 0.012$ & 0.736 \\
\hline 217 & $902-52409-135$ & J094903.36+500126.0 & $1.091 \pm 0.037$ & $8.256 \pm 0.034$ & 8.608 & $-1.058 \pm 0.006$ & $-0.844 \pm 0.008$ & $1.816 \pm 0.004$ & 0.743 \\
\hline 218 & $903-52400-600$ & $\mathrm{~J} 100751.67+525624.0$ & $1.216 \pm 0.050$ & $8.105 \pm 0.034$ & 8.076 & $-1.396 \pm 0.017$ & $-0.802 \pm 0.008$ & $2.070 \pm 0.014$ & 0.648 \\
\hline 219 & 904- 52381- 501 & $\mathrm{~J} 102024.05+540043.9$ & $1.078 \pm 0.044$ & $8.272 \pm 0.045$ & 8.162 & $-1.374 \pm 0.011$ & $-0.907 \pm 0.009$ & $2.162 \pm 0.008$ & 0.780 \\
\hline 220 & 908- 52373- 27 & $\mathrm{~J} 111558.18+554806.4$ & $1.175 \pm 0.037$ & $8.166 \pm 0.028$ & 8.334 & $-1.237 \pm 0.007$ & $-0.800 \pm 0.006$ & $1.946 \pm 0.004$ & 0.667 \\
\hline 221 & 909-52379- 616 & $\mathrm{~J} 112313.30+570327.7$ & $1.362 \pm 0.027$ & $7.966 \pm 0.021$ & 8.004 & $-1.747 \pm 0.022$ & $-1.072 \pm 0.010$ & $2.562 \pm 0.020$ & 0.823 \\
\hline 222 & 915- 52443- 598 & $\mathrm{~J} 140539.17-015334.4$ & $1.160 \pm 0.068$ & $8.192 \pm 0.054$ & 8.146 & $-1.312 \pm 0.011$ & $-0.818 \pm 0.008$ & $2.058 \pm 0.007$ & 0.699 \\
\hline 223 & 926- 52413- 279 & $\mathrm{~J} 153804.80-014940.8$ & $1.072 \pm 0.063$ & $8.320 \pm 0.060$ & 8.352 & $-1.294 \pm 0.008$ & $-0.860 \pm 0.007$ & $2.074 \pm 0.006$ & 0.716 \\
\hline 224 & $927-52577-514$ & $\mathrm{~J} 074553.04+231017.0$ & $1.080 \pm 0.048$ & $8.248 \pm 0.041$ & 8.681 & $-0.981 \pm 0.007$ & $-0.838 \pm 0.011$ & $1.669 \pm 0.006$ & 0.681 \\
\hline 225 & 934- 52672- 483 & J084725.06+355451.4 & $1.069 \pm 0.025$ & $8.326 \pm 0.025$ & 8.570 & $-1.248 \pm 0.008$ & $-0.923 \pm 0.008$ & $2.071 \pm 0.006$ & 0.771 \\
\hline 226 & 936- 52705- 477 & J085949.13+380632.0 & $1.260 \pm 0.032$ & $8.113 \pm 0.027$ & 8.118 & $-1.512 \pm 0.014$ & $-0.988 \pm 0.010$ & $2.384 \pm 0.012$ & 0.830 \\
\hline 227 & 937- 52707- 275 & $\mathrm{~J} 090741.52+385202.2$ & $1.107 \pm 0.066$ & $8.212 \pm 0.055$ & 8.610 & $-1.141 \pm 0.009$ & $-0.797 \pm 0.009$ & $1.809 \pm 0.006$ & 0.659 \\
\hline 228 & $940-52670-594$ & J094330.67+422142.8 & $1.013 \pm 0.058$ & $8.367 \pm 0.059$ & 8.429 & $-1.116 \pm 0.009$ & $-0.730 \pm 0.008$ & $1.836 \pm 0.007$ & 0.680 \\
\hline 229 & $945-52652-423$ & $\mathrm{~J} 095859.35+552415.8$ & $1.161 \pm 0.040$ & $8.184 \pm 0.033$ & 8.214 & $-1.266 \pm 0.010$ & $-0.808 \pm 0.007$ & $2.046 \pm 0.008$ & 0.752 \\
\hline 230 & 949- 52427- 359 & $\mathrm{~J} 104337.66+580820.7$ & $1.235 \pm 0.040$ & $8.322 \pm 0.076$ & 8.049 & $-1.497 \pm 0.013$ & $-0.996 \pm 0.010$ & $2.291 \pm 0.011$ & 0.523 \\
\hline 231 & 952- 52409- 264 & $\mathrm{~J} 112509.46+584700.9$ & $1.004 \pm 0.046$ & $8.360 \pm 0.046$ & 8.612 & $-1.153 \pm 0.007$ & $-0.839 \pm 0.006$ & $1.842 \pm 0.004$ & 0.667 \\
\hline 232 & $952-52409-439$ & $\mathrm{~J} 112555.10+593319.0$ & $1.235 \pm 0.025$ & $8.157 \pm 0.016$ & 8.197 & $-1.347 \pm 0.009$ & $-0.817 \pm 0.006$ & $2.133 \pm 0.007$ & 0.695 \\
\hline 233 & 952- 52409- 447 & $\mathrm{~J} 113116.49+601229.5$ & $1.105 \pm 0.058$ & $8.224 \pm 0.053$ & 8.103 & $-1.343 \pm 0.013$ & $-0.871 \pm 0.011$ & $2.098 \pm 0.011$ & 0.753 \\
\hline 234 & $955-52409-383$ & $\mathrm{~J} 121142.86+603626.6$ & $1.166 \pm 0.054$ & $8.204 \pm 0.045$ & 8.235 & $-1.295 \pm 0.013$ & $-0.823 \pm 0.012$ & $2.121 \pm 0.011$ & 0.779 \\
\hline 235 & 956- 52401- 20 & $\mathrm{~J} 124340.29+590311.1$ & $1.071 \pm 0.070$ & $8.309 \pm 0.063$ & 8.258 & $-1.234 \pm 0.015$ & $-0.759 \pm 0.009$ & $1.950 \pm 0.013$ & 0.652 \\
\hline 236 & 956- 52401- 347 & $\mathrm{~J} 122630.60+595712.6$ & $1.126 \pm 0.063$ & $8.235 \pm 0.057$ & 8.144 & $-1.429 \pm 0.024$ & $-0.930 \pm 0.013$ & $2.240 \pm 0.022$ & 0.778 \\
\hline 237 & 957- 52398- 210 & $\mathrm{~J} 125328.61+584021.7$ & $1.114 \pm 0.027$ & $8.272 \pm 0.027$ & 8.306 & $-1.375 \pm 0.009$ & $-0.930 \pm 0.007$ & $2.211 \pm 0.006$ & 0.785 \\
\hline 238 & 957- 52398- 530 & $\mathrm{~J} 125856.26+604730.8$ & $1.074 \pm 0.045$ & $8.289 \pm 0.044$ & 8.297 & $-1.319 \pm 0.012$ & $-0.889 \pm 0.009$ & $2.105 \pm 0.009$ & 0.759 \\
\hline 239 & 959-52411- 137 & $\mathrm{~J} 132455.03+574510.7$ & $1.163 \pm 0.028$ & $8.219 \pm 0.028$ & 8.760 & $-1.281 \pm 0.009$ & $-1.056 \pm 0.010$ & $2.152 \pm 0.007$ & 0.824 \\
\hline 240 & $963-52643-165$ & $\mathrm{~J} 105315.89+471517.2$ & $1.289 \pm 0.057$ & $8.083 \pm 0.034$ & 8.472 & $-1.127 \pm 0.009$ & $-0.748 \pm 0.008$ & $1.815 \pm 0.006$ & 0.608 \\
\hline 241 & 964- 52646- 570 & J110918.05+494753.8 & $1.109 \pm 0.030$ & $8.249 \pm 0.025$ & 8.453 & $-1.169 \pm 0.007$ & $-0.780 \pm 0.006$ & $1.890 \pm 0.005$ & 0.674 \\
\hline 242 & 969- 52442- 354 & $\mathrm{~J} 115630.62+500822.2$ & $1.156 \pm 0.036$ & $8.147 \pm 0.029$ & 8.764 & $-1.040 \pm 0.007$ & $-0.950 \pm 0.013$ & $1.772 \pm 0.005$ & 0.748 \\
\hline 243 & 971- 52644- 599 & $\mathrm{~J} 123153.64+500056.5$ & $1.105 \pm 0.057$ & $8.192 \pm 0.048$ & 8.673 & $-1.000 \pm 0.007$ & $-0.824 \pm 0.010$ & $1.664 \pm 0.004$ & 0.680 \\
\hline 244 & $972-52435-370$ & $\mathrm{~J} 165844.50+351923.1$ & $1.108 \pm 0.035$ & $8.251 \pm 0.030$ & 8.329 & $-1.248 \pm 0.008$ & $-0.822 \pm 0.007$ & $2.011 \pm 0.006$ & 0.724 \\
\hline 245 & $978-52441-408$ & $\mathrm{~J} 171400.93+313023.4$ & $1.053 \pm 0.043$ & $8.309 \pm 0.039$ & 8.621 & $-1.032 \pm 0.007$ & $-0.841 \pm 0.013$ & $1.746 \pm 0.006$ & 0.681 \\
\hline 246 & $979-52427-83$ & $\mathrm{~J} 172006.75+255827.4$ & $1.110 \pm 0.066$ & $8.223 \pm 0.054$ & 8.219 & $-1.214 \pm 0.014$ & $-0.760 \pm 0.010$ & $1.903 \pm 0.012$ & 0.664 \\
\hline 247 & 984- 52442- 263 & $\mathrm{~J} 205510.41-001903.0$ & $1.063 \pm 0.065$ & $8.235 \pm 0.053$ & 8.740 & $-0.812 \pm 0.005$ & $-0.709 \pm 0.006$ & $1.354 \pm 0.004$ & 0.558 \\
\hline 248 & 987- 52523- 394 & J211958.32+005233.6 & $1.145 \pm 0.039$ & $8.235 \pm 0.037$ & 8.138 & $-1.449 \pm 0.012$ & $-0.947 \pm 0.009$ & $2.303 \pm 0.010$ & 0.806 \\
\hline 249 & 987- 52523- 440 & J212043.97+010006.8 & $1.025 \pm 0.058$ & $8.335 \pm 0.055$ & 8.513 & $-1.064 \pm 0.007$ & $-0.722 \pm 0.006$ & $1.722 \pm 0.002$ & 0.624 \\
\hline 250 & 988- 52520- 456 & J212829.71+003021.9 & $1.100 \pm 0.067$ & $8.229 \pm 0.053$ & 8.274 & $-1.139 \pm 0.009$ & $-0.664 \pm 0.007$ & $1.753 \pm 0.006$ & 0.588 \\
\hline 251 & 992- 52644- 19 & $\mathrm{~J} 093625.35+050332.0$ & $1.362 \pm 0.036$ & $8.015 \pm 0.028$ & 8.118 & $-1.516 \pm 0.013$ & $-1.050 \pm 0.014$ & $2.396 \pm 0.011$ & 0.844 \\
\hline 252 & 997- 52734- 474 & $\mathrm{~J} 101637.77+065214.5$ & $1.184 \pm 0.079$ & $8.140 \pm 0.060$ & 8.078 & $-1.375 \pm 0.017$ & $-0.793 \pm 0.011$ & $2.069 \pm 0.014$ & 0.668 \\
\hline 253 & 999- 52636- 150 & $\mathrm{~J} 103404.17+061210.4$ & $1.156 \pm 0.036$ & $8.185 \pm 0.030$ & 8.587 & $-1.217 \pm 0.008$ & $-0.911 \pm 0.007$ & $1.991 \pm 0.006$ & 0.754 \\
\hline 254 & $1001-52670-385$ & $\mathrm{~J} 104727.84+072238.2$ & $0.983 \pm 0.055$ & $8.342 \pm 0.060$ & 8.847 & $-0.909 \pm 0.007$ & $-0.919 \pm 0.016$ & $1.587 \pm 0.005$ & 0.717 \\
\hline 255 & $1001-52670-611$ & $\mathrm{~J} 105445.74+073731.8$ & $1.127 \pm 0.058$ & $8.230 \pm 0.047$ & 8.479 & $-1.173 \pm 0.037$ & $-0.836 \pm 0.014$ & $1.897 \pm 0.035$ & 0.677 \\
\hline 256 & $1002-52646-525$ & $\mathrm{~J} 105854.79+080043.9$ & $0.917 \pm 0.051$ & $8.503 \pm 0.064$ & 8.578 & $-1.198 \pm 0.008$ & $-0.851 \pm 0.008$ & $1.931 \pm 0.006$ & 0.711 \\
\hline 257 & $1005-52703-268$ & $\mathrm{~J} 094422.13+480130.3$ & $1.446 \pm 0.045$ & $7.958 \pm 0.033$ & 8.055 & $-1.813 \pm 0.027$ & $-1.169 \pm 0.016$ & $2.721 \pm 0.023$ & 0.866 \\
\hline 258 & $1007-52706-225$ & $\mathrm{~J} 100912.50+500604.6$ & $1.339 \pm 0.108$ & $7.910 \pm 0.063$ & 8.660 & $-0.946 \pm 0.009$ & $-0.746 \pm 0.010$ & $1.532 \pm 0.006$ & 0.636 \\
\hline 259 & $1008-52707-430$ & $\mathrm{~J} 101855.46+515527.8$ & $1.267 \pm 0.033$ & $8.097 \pm 0.024$ & 8.153 & $-1.418 \pm 0.011$ & $-0.932 \pm 0.012$ & $2.232 \pm 0.009$ & 0.765 \\
\hline 260 & $1013-52707-229$ & $\mathrm{~J} 111824.48+540115.9$ & $1.188 \pm 0.086$ & $8.226 \pm 0.071$ & 8.166 & $-1.562 \pm 0.027$ & $-1.002 \pm 0.019$ & $2.439 \pm 0.024$ & 0.785 \\
\hline 261 & 1014- 52707- 393 & $\mathrm{~J} 112544.09+550532.2$ & $1.039 \pm 0.066$ & $8.275 \pm 0.062$ & 8.688 & $-0.990 \pm 0.007$ & $-0.815 \pm 0.008$ & $1.631 \pm 0.005$ & 0.656 \\
\hline 262 & $1020-52721-287$ & $\mathrm{~J} 122842.45+535723.0$ & $1.183 \pm 0.052$ & $8.166 \pm 0.047$ & 8.131 & $-1.455 \pm 0.014$ & $-0.965 \pm 0.011$ & $2.308 \pm 0.012$ & 0.842 \\
\hline 263 & $1021-52460-446$ & $\mathrm{~J} 204018.05+010324.4$ & $1.075 \pm 0.064$ & $8.253 \pm 0.058$ & 8.536 & $-1.156 \pm 0.009$ & $-0.782 \pm 0.007$ & $1.871 \pm 0.007$ & 0.714 \\
\hline
\end{tabular}


Table 1. continued.

\begin{tabular}{|c|c|c|c|c|c|c|c|c|c|}
\hline (1) & (2) & (3) & (4) & (5) & (6) & (7) & (8) & (9) & (10) \\
\hline Num & Plate-MJD-FiberID & IAU designations & $t_{3}\left(10^{4} \mathrm{~K}\right)$ & $12+\log (\mathrm{O} / \mathrm{H})_{T_{\mathrm{e}}}$ & $(\mathrm{O} / \mathrm{H})_{\text {Bay }}$ & $\mathrm{N} 2$ & S2 & O3N2 & $P$ \\
\hline 264 & 1024- 52826- 106 & J210134.44-002846.9 & $1.401 \pm 0.099$ & $7.982 \pm 0.056$ & 8.150 & $-1.370 \pm 0.027$ & $-0.824 \pm 0.024$ & $2.159 \pm 0.025$ & 0.726 \\
\hline 265 & 1026- 52558- 122 & $\mathrm{~J} 211451.48-011046.2$ & $1.142 \pm 0.062$ & $8.210 \pm 0.051$ & 8.109 & $-1.378 \pm 0.018$ & $-0.823 \pm 0.009$ & $2.133 \pm 0.016$ & 0.714 \\
\hline 266 & $1026-52558-596$ & $\mathrm{~J} 211829.85+003059.4$ & $1.262 \pm 0.041$ & $8.102 \pm 0.029$ & 8.395 & $-1.320 \pm 0.017$ & $-0.904 \pm 0.013$ & $2.136 \pm 0.015$ & 0.771 \\
\hline 267 & $1028-52562-373$ & $\mathrm{~J} 212705.80+005827.1$ & $1.362 \pm 0.041$ & $8.011 \pm 0.027$ & 8.079 & $-1.647 \pm 0.023$ & $-0.993 \pm 0.011$ & $2.475 \pm 0.021$ & 0.781 \\
\hline 268 & $1028-52562-388$ & J212702.90+002701.8 & $1.011 \pm 0.056$ & $8.357 \pm 0.059$ & 8.464 & $-1.256 \pm 0.012$ & $-0.872 \pm 0.009$ & $2.002 \pm 0.010$ & 0.728 \\
\hline 269 & $1028-52562-422$ & $\mathrm{~J} 212829.71+003021.9$ & $1.004 \pm 0.061$ & $8.351 \pm 0.058$ & 8.187 & $-1.139 \pm 0.008$ & $-0.645 \pm 0.006$ & $1.757 \pm 0.006$ & 0.593 \\
\hline 270 & $1028-52562-592$ & $\mathrm{~J} 213338.95+002605.2$ & $1.334 \pm 0.038$ & $8.024 \pm 0.025$ & 8.074 & $-1.610 \pm 0.018$ & $-0.961 \pm 0.009$ & $2.409 \pm 0.015$ & 0.758 \\
\hline 271 & 1028- 52884- 386 & $\mathrm{~J} 212705.80+005827.1$ & $1.291 \pm 0.040$ & $8.071 \pm 0.028$ & 8.125 & $-1.552 \pm 0.020$ & $-0.951 \pm 0.010$ & $2.368 \pm 0.018$ & 0.772 \\
\hline 272 & 1028- 52884- 562 & $\mathrm{~J} 213338.95+002605.2$ & $1.328 \pm 0.032$ & $8.029 \pm 0.022$ & 8.084 & $-1.597 \pm 0.015$ & $-0.962 \pm 0.008$ & $2.409 \pm 0.012$ & 0.776 \\
\hline 273 & 1030- 52914- 77 & $\mathrm{~J} 214642.29+000009.0$ & $1.076 \pm 0.047$ & $8.498 \pm 0.057$ & 8.227 & $-1.420 \pm 0.010$ & $-0.957 \pm 0.009$ & $2.248 \pm 0.008$ & 0.562 \\
\hline 274 & 1030- 52914- 107 & J214459.59-001140.2 & $1.003 \pm 0.050$ & $8.355 \pm 0.051$ & 8.441 & $-1.242 \pm 0.009$ & $-0.829 \pm 0.006$ & $1.946 \pm 0.007$ & 0.693 \\
\hline 275 & $1032-53175-635$ & $\mathrm{~J} 220412.46+002201.5$ & $1.328 \pm 0.058$ & $8.045 \pm 0.045$ & 8.058 & $-1.512 \pm 0.019$ & $-0.862 \pm 0.011$ & $2.356 \pm 0.012$ & 0.795 \\
\hline 276 & $1033-52822-458$ & $\mathrm{~J} 220707.90+004658.8$ & $1.306 \pm 0.097$ & $8.035 \pm 0.064$ & 8.102 & $-1.473 \pm 0.031$ & $-0.865 \pm 0.015$ & $2.269 \pm 0.029$ & 0.773 \\
\hline 277 & $1034-52525-551$ & $\mathrm{~J} 221549.25+010938.8$ & $1.197 \pm 0.053$ & $8.141 \pm 0.040$ & 8.110 & $-1.341 \pm 0.014$ & $-0.818 \pm 0.008$ & $2.092 \pm 0.012$ & 0.722 \\
\hline 278 & 1034- 52813- 521 & J221549.25+010938.8 & $1.091 \pm 0.047$ & $8.273 \pm 0.041$ & 8.095 & $-1.372 \pm 0.011$ & $-0.816 \pm 0.006$ & $2.123 \pm 0.009$ & 0.706 \\
\hline 279 & 1035- 52816- 574 & $\mathrm{~J} 222358.95+005007.8$ & $1.217 \pm 0.037$ & $8.170 \pm 0.032$ & 8.301 & $-1.310 \pm 0.046$ & $-1.008 \pm 0.012$ & $2.202 \pm 0.044$ & 0.840 \\
\hline 280 & $1040-52722-21$ & $\mathrm{~J} 132150.45+534127.9$ & $1.221 \pm 0.061$ & $8.127 \pm 0.047$ & 8.083 & $-1.545 \pm 0.016$ & $-0.908 \pm 0.011$ & $2.335 \pm 0.014$ & 0.755 \\
\hline 281 & $1040-52722-358$ & $\mathrm{~J} 130728.44+542652.4$ & $1.123 \pm 0.061$ & $8.204 \pm 0.045$ & 8.166 & $-1.172 \pm 0.007$ & $-0.702 \pm 0.006$ & $1.702 \pm 0.001$ & 0.501 \\
\hline 282 & $1042-52725-399$ & $\mathrm{~J} 133446.30+534927.4$ & $1.172 \pm 0.052$ & $8.162 \pm 0.046$ & 8.768 & $-1.319 \pm 0.017$ & $-1.098 \pm 0.030$ & $2.146 \pm 0.015$ & 0.827 \\
\hline 283 & 1043- 52465- 78 & $\mathrm{~J} 135624.07+523851.0$ & $1.203 \pm 0.077$ & $8.095 \pm 0.058$ & 8.613 & $-1.191 \pm 0.013$ & $-0.880 \pm 0.014$ & $1.896 \pm 0.011$ & 0.712 \\
\hline 284 & $1052-52466-115$ & $\mathrm{~J} 153808.50+431421.1$ & $1.164 \pm 0.078$ & $8.174 \pm 0.057$ & 8.530 & $-1.067 \pm 0.009$ & $-0.684 \pm 0.007$ & $1.708 \pm 0.006$ & 0.595 \\
\hline 285 & 1054- 52516- 499 & $\mathrm{~J} 155411.09+400602.8$ & $1.098 \pm 0.067$ & $8.207 \pm 0.061$ & 8.058 & $-1.299 \pm 0.012$ & $-0.769 \pm 0.009$ & $2.001 \pm 0.009$ & 0.718 \\
\hline 286 & 1060- 52636- 21 & J075638.45+291819.8 & $1.342 \pm 0.090$ & $7.944 \pm 0.048$ & 8.690 & $-0.834 \pm 0.007$ & $-0.731 \pm 0.014$ & $1.386 \pm 0.005$ & 0.557 \\
\hline 287 & $1060-52636-328$ & $\mathrm{~J} 075230.29+301607.6$ & $1.247 \pm 0.046$ & $8.076 \pm 0.035$ & 8.037 & $-1.556 \pm 0.031$ & $-0.943 \pm 0.013$ & $2.341 \pm 0.028$ & 0.778 \\
\hline 288 & $1063-52591-389$ & $\mathrm{~J} 033128.49+003737.5$ & $1.599 \pm 0.082$ & $7.789 \pm 0.039$ & 8.086 & $-1.439 \pm 0.025$ & $-0.809 \pm 0.013$ & $2.172 \pm 0.019$ & 0.715 \\
\hline 289 & 1064- 52577- 509 & $\mathrm{~J} 032713.08+003112.3$ & $1.184 \pm 0.062$ & $8.171 \pm 0.050$ & 8.212 & $-1.320 \pm 0.016$ & $-0.859 \pm 0.011$ & $2.124 \pm 0.014$ & 0.766 \\
\hline 290 & 1067- 52616- 112 & J030502.28-000453.7 & $1.169 \pm 0.059$ & $8.161 \pm 0.051$ & 8.041 & $-1.478 \pm 0.016$ & $-0.935 \pm 0.011$ & $2.268 \pm 0.013$ & 0.783 \\
\hline 291 & 1069- 52590- 399 & J024359.02+003322.6 & $1.304 \pm 0.036$ & $8.075 \pm 0.028$ & 8.108 & $-1.606 \pm 0.020$ & $-1.053 \pm 0.012$ & $2.483 \pm 0.018$ & 0.830 \\
\hline 292 & 1073- 52649- 409 & J021306.62+005612.4 & $1.427 \pm 0.018$ & $7.972 \pm 0.021$ & 8.654 & $-1.555 \pm 0.007$ & $-1.259 \pm 0.007$ & $2.501 \pm 0.005$ & 0.921 \\
\hline 293 & 1073- 52649- 419 & $\mathrm{~J} 021332.93+010825.8$ & $1.419 \pm 0.042$ & $7.973 \pm 0.030$ & 8.045 & $-1.699 \pm 0.023$ & $-1.047 \pm 0.013$ & $2.602 \pm 0.020$ & 0.868 \\
\hline 294 & 1074- 52937- 186 & J020551.72-003229.0 & $1.021 \pm 0.057$ & $8.292 \pm 0.054$ & 8.602 & $-1.022 \pm 0.006$ & $-0.700 \pm 0.006$ & $1.627 \pm 0.004$ & 0.620 \\
\hline 295 & $1074-52937-573$ & J020817.62+004358.4 & $1.145 \pm 0.045$ & $8.214 \pm 0.040$ & 8.182 & $-1.440 \pm 0.014$ & $-0.995 \pm 0.011$ & $2.252 \pm 0.012$ & 0.777 \\
\hline 296 & $1081-52531-17$ & J011640.25-004712.8 & $1.257 \pm 0.075$ & $8.141 \pm 0.050$ & 8.264 & $-1.355 \pm 0.018$ & $-0.874 \pm 0.014$ & $2.140 \pm 0.016$ & 0.696 \\
\hline 297 & 1083- 52520- 354 & J005410.34+003812.4 & $1.259 \pm 0.078$ & $8.120 \pm 0.059$ & 8.143 & $-1.546 \pm 0.036$ & $-1.109 \pm 0.026$ & $2.443 \pm 0.033$ & 0.858 \\
\hline 298 & 1084- 52591- 99 & J005132.11-004608.0 & $1.343 \pm 0.097$ & $7.957 \pm 0.062$ & 7.923 & $-2.024 \pm 0.070$ & $-1.082 \pm 0.019$ & $2.792 \pm 0.068$ & 0.794 \\
\hline 299 & 1093- 52591- 261 & J233817.90-001157.4 & $1.212 \pm 0.071$ & $8.175 \pm 0.054$ & 8.268 & $-1.338 \pm 0.013$ & $-0.889 \pm 0.009$ & $2.154 \pm 0.010$ & 0.739 \\
\hline 300 & $1094-52524-543$ & $\mathrm{~J} 233549.15+001314.8$ & $1.426 \pm 0.069$ & $7.887 \pm 0.043$ & 7.882 & $-1.746 \pm 0.061$ & $-1.284 \pm 0.054$ & $2.558 \pm 0.059$ & 0.853 \\
\hline 301 & $1095-52521-381$ & $\mathrm{~J} 232122.52+003455.2$ & $1.217 \pm 0.071$ & $8.154 \pm 0.057$ & 8.122 & $-1.558 \pm 0.029$ & $-0.988 \pm 0.018$ & $2.408 \pm 0.025$ & 0.803 \\
\hline 302 & $1095-52521-619$ & $\mathrm{~J} 232757.74+005819.2$ & $1.279 \pm 0.096$ & $8.067 \pm 0.067$ & 8.075 & $-1.596 \pm 0.034$ & $-0.940 \pm 0.017$ & $2.394 \pm 0.031$ & 0.770 \\
\hline 303 & 1096- 52974- 102 & $\mathrm{~J} 231845.21-002610.3$ & $1.124 \pm 0.057$ & $8.224 \pm 0.046$ & 8.147 & $-1.297 \pm 0.009$ & $-0.784 \pm 0.012$ & $2.035 \pm 0.007$ & 0.707 \\
\hline 304 & $1096-52974-630$ & $\mathrm{~J} 232122.52+003455.2$ & $1.173 \pm 0.061$ & $8.204 \pm 0.052$ & 8.103 & $-1.626 \pm 0.024$ & $-1.005 \pm 0.013$ & $2.483 \pm 0.021$ & 0.811 \\
\hline 305 & $1101-52621-576$ & $\mathrm{~J} 224041.33+005703.6$ & $1.257 \pm 0.073$ & $8.145 \pm 0.052$ & 8.334 & $-1.400 \pm 0.027$ & $-0.986 \pm 0.021$ & $2.265 \pm 0.025$ & 0.785 \\
\hline 306 & $1104-52912-439$ & $\mathrm{~J} 221243.06+000648.6$ & $1.141 \pm 0.061$ & $8.221 \pm 0.053$ & 8.505 & $-1.278 \pm 0.035$ & $-0.965 \pm 0.024$ & $2.102 \pm 0.033$ & 0.806 \\
\hline 307 & $1104-52912-511$ & J221523.06+000246.6 & $1.304 \pm 0.019$ & $8.093 \pm 0.020$ & 8.711 & $-1.350 \pm 0.007$ & $-1.162 \pm 0.010$ & $2.280 \pm 0.005$ & 0.877 \\
\hline 308 & 1116- 52932- 12 & J205034.66-004619.9 & $1.486 \pm 0.095$ & $7.867 \pm 0.045$ & 8.000 & $-1.528 \pm 0.032$ & $-0.902 \pm 0.021$ & $2.213 \pm 0.029$ & 0.671 \\
\hline 309 & 1116- 52932- 89 & J204827.46-005958.5 & $1.615 \pm 0.060$ & $7.723 \pm 0.030$ & 7.896 & $-1.828 \pm 0.028$ & $-1.089 \pm 0.013$ & $2.574 \pm 0.026$ & 0.801 \\
\hline 310 & $1156-52641-378$ & $\mathrm{~J} 033128.49+003737.5$ & $1.392 \pm 0.065$ & $7.919 \pm 0.037$ & 7.991 & $-1.506 \pm 0.019$ & $-0.891 \pm 0.011$ & $2.220 \pm 0.017$ & 0.720 \\
\hline 311 & $1156-52641-423$ & $\mathrm{~J} 033047.69+002920.4$ & $1.166 \pm 0.048$ & $8.187 \pm 0.038$ & 8.176 & $-1.360 \pm 0.012$ & $-0.880 \pm 0.009$ & $2.125 \pm 0.010$ & 0.724 \\
\hline 312 & $1158-52668-370$ & $\mathrm{~J} 135013.80+585313.5$ & $1.175 \pm 0.085$ & $8.113 \pm 0.064$ & 8.217 & $-1.160 \pm 0.011$ & $-0.716 \pm 0.010$ & $1.774 \pm 0.008$ & 0.619 \\
\hline 313 & $1160-52674-534$ & $\mathrm{~J} 141652.46+571215.8$ & $1.103 \pm 0.057$ & $8.223 \pm 0.048$ & 8.157 & $-1.212 \pm 0.012$ & $-0.719 \pm 0.011$ & $1.902 \pm 0.010$ & 0.675 \\
\hline 314 & $1163-52669-284$ & $\mathrm{~J} 144328.47+533919.0$ & $1.221 \pm 0.031$ & $8.121 \pm 0.024$ & 8.577 & $-1.259 \pm 0.008$ & $-0.925 \pm 0.007$ & $2.049 \pm 0.006$ & 0.764 \\
\hline 315 & $1168-52731-639$ & $\mathrm{~J} 155548.46+465715.8$ & $1.243 \pm 0.078$ & $8.080 \pm 0.055$ & 8.135 & $-1.238 \pm 0.013$ & $-0.697 \pm 0.009$ & $1.913 \pm 0.010$ & 0.644 \\
\hline 316 & $1170-52756-485$ & $\mathrm{~J} 160821.94+432738.5$ & $1.041 \pm 0.064$ & $8.309 \pm 0.063$ & 8.579 & $-1.157 \pm 0.009$ & $-0.815 \pm 0.011$ & $1.882 \pm 0.007$ & 0.714 \\
\hline
\end{tabular}


Table 1. continued.

\begin{tabular}{|c|c|c|c|c|c|c|c|c|c|}
\hline (1) & (2) & (3) & (4) & (5) & (6) & (7) & (8) & (9) & (10) \\
\hline Num & Plate-MJD-FiberID & IAU designations & $t_{3}\left(10^{4} \mathrm{~K}\right)$ & $12+\log (\mathrm{O} / \mathrm{H})_{T_{\mathrm{e}}}$ & $(\mathrm{O} / \mathrm{H})_{\text {Bay }}$ & $\mathrm{N} 2$ & S2 & $\mathrm{O} 3 \mathrm{~N} 2$ & $P$ \\
\hline 317 & 1176- 52791- 591 & $\mathrm{~J} 165712.75+321141.2$ & $1.208 \pm 0.031$ & $8.094 \pm 0.025$ & 8.097 & $-1.344 \pm 0.008$ & $-0.894 \pm 0.007$ & $2.085 \pm 0.006$ & 0.751 \\
\hline 318 & $1177-52824-556$ & J214350.86-072003.8 & $1.388 \pm 0.044$ & $7.950 \pm 0.028$ & 8.551 & $-1.299 \pm 0.013$ & $-0.957 \pm 0.011$ & $2.097 \pm 0.011$ & 0.793 \\
\hline 319 & $1177-52824-616$ & $\mathrm{~J} 214338.30-065034.8$ & $1.155 \pm 0.061$ & $8.179 \pm 0.048$ & 8.221 & $-1.215 \pm 0.009$ & $-0.755 \pm 0.009$ & $1.912 \pm 0.005$ & 0.665 \\
\hline 320 & 1184- 52641- 581 & J082057.60+034503.6 & $1.049 \pm 0.064$ & $8.296 \pm 0.058$ & 8.584 & $-1.091 \pm 0.007$ & $-0.735 \pm 0.006$ & $1.740 \pm 0.005$ & 0.624 \\
\hline 321 & $1192-52649-173$ & J090356.79+045613.5 & $1.238 \pm 0.041$ & $8.086 \pm 0.031$ & 8.411 & $-1.310 \pm 0.022$ & $-0.948 \pm 0.011$ & $2.093 \pm 0.019$ & 0.775 \\
\hline 322 & 1195- 52724- 60 & J092540.94+063116.6 & $1.198 \pm 0.040$ & $8.163 \pm 0.031$ & 8.112 & $-1.419 \pm 0.011$ & $-0.862 \pm 0.008$ & $2.204 \pm 0.008$ & 0.734 \\
\hline 323 & $1199-52703-600$ & J090610.83+395335.1 & $1.170 \pm 0.050$ & $8.171 \pm 0.035$ & 8.278 & $-1.151 \pm 0.009$ & $-0.679 \pm 0.006$ & $1.805 \pm 0.006$ & 0.608 \\
\hline 324 & $1203-52669-570$ & $\mathrm{~J} 074915.48+225342.3$ & $0.983 \pm 0.054$ & $8.417 \pm 0.056$ & 8.539 & $-1.163 \pm 0.008$ & $-0.777 \pm 0.007$ & $1.850 \pm 0.005$ & 0.635 \\
\hline 325 & 1205- 52670- 307 & $\mathrm{~J} 075838.40+252558.4$ & $1.018 \pm 0.055$ & $8.265 \pm 0.052$ & 8.797 & $-0.938 \pm 0.006$ & $-0.873 \pm 0.007$ & $1.526 \pm 0.004$ & 0.640 \\
\hline 326 & $1207-52672-506$ & J082527.65+295739.2 & $1.001 \pm 0.048$ & $8.368 \pm 0.046$ & 8.346 & $-1.129 \pm 0.007$ & $-0.679 \pm 0.006$ & $1.761 \pm 0.005$ & 0.597 \\
\hline 327 & $1212-52703-388$ & J090403.60+363914.0 & $1.137 \pm 0.036$ & $8.238 \pm 0.030$ & 8.124 & $-1.411 \pm 0.011$ & $-0.854 \pm 0.008$ & $2.178 \pm 0.008$ & 0.702 \\
\hline 328 & $1212-52703-584$ & J091208.95+362226.4 & $1.281 \pm 0.029$ & $8.061 \pm 0.022$ & 8.641 & $-1.230 \pm 0.009$ & $-0.993 \pm 0.011$ & $2.033 \pm 0.007$ & 0.781 \\
\hline 329 & $1213-52972-88$ & $\mathrm{~J} 091724.07+362618.9$ & $0.974 \pm 0.050$ & $8.397 \pm 0.053$ & 8.552 & $-1.152 \pm 0.008$ & $-0.773 \pm 0.007$ & $1.824 \pm 0.005$ & 0.653 \\
\hline 330 & $1213-52972-500$ & J091640.05+373159.5 & $1.277 \pm 0.039$ & $8.105 \pm 0.028$ & 8.136 & $-1.464 \pm 0.015$ & $-0.903 \pm 0.010$ & $2.289 \pm 0.013$ & 0.758 \\
\hline 331 & 1214- 52731- 339 & J092126.45+384619.2 & $0.996 \pm 0.047$ & $8.344 \pm 0.050$ & 8.653 & $-1.050 \pm 0.007$ & $-0.879 \pm 0.008$ & $1.753 \pm 0.005$ & 0.721 \\
\hline 332 & $1215-52725-273$ & J093538.86+383754.4 & $1.187 \pm 0.024$ & $8.205 \pm 0.025$ & 8.786 & $-1.440 \pm 0.011$ & $-1.131 \pm 0.013$ & $2.345 \pm 0.009$ & 0.859 \\
\hline 333 & $1215-52725-629$ & J094314.40+403842.7 & $1.078 \pm 0.062$ & $8.282 \pm 0.051$ & 8.581 & $-1.035 \pm 0.008$ & $-0.682 \pm 0.007$ & $1.657 \pm 0.005$ & 0.569 \\
\hline 334 & $1217-52672-271$ & J095538.93+414320.6 & $1.040 \pm 0.065$ & $8.328 \pm 0.064$ & 8.111 & $-1.341 \pm 0.013$ & $-0.830 \pm 0.009$ & $2.083 \pm 0.010$ & 0.709 \\
\hline 335 & 1223- 52781- 287 & $\mathrm{~J} 112437.80+083112.7$ & $1.237 \pm 0.069$ & $8.074 \pm 0.047$ & 8.573 & $-1.098 \pm 0.009$ & $-0.739 \pm 0.007$ & $1.773 \pm 0.006$ & 0.661 \\
\hline 336 & 1224- 52765- 566 & $\mathrm{~J} 114025.94+104653.7$ & $1.191 \pm 0.074$ & $8.167 \pm 0.056$ & 8.290 & $-1.252 \pm 0.014$ & $-0.804 \pm 0.013$ & $2.012 \pm 0.011$ & 0.708 \\
\hline 337 & $1233-52734-335$ & $\mathrm{~J} 123444.26+104308.7$ & $1.219 \pm 0.033$ & $8.158 \pm 0.026$ & 8.119 & $-1.494 \pm 0.011$ & $-0.905 \pm 0.007$ & $2.296 \pm 0.007$ & 0.736 \\
\hline 338 & $1237-52762-42$ & $\mathrm{~J} 101629.88+073404.8$ & $1.173 \pm 0.035$ & $8.211 \pm 0.029$ & 8.722 & $-1.088 \pm 0.008$ & $-0.922 \pm 0.012$ & $1.920 \pm 0.007$ & 0.774 \\
\hline 339 & $1240-52734-340$ & $\mathrm{~J} 103509.33+094516.9$ & $1.070 \pm 0.039$ & $8.263 \pm 0.039$ & 8.596 & $-1.207 \pm 0.009$ & $-0.914 \pm 0.009$ & $1.962 \pm 0.006$ & 0.761 \\
\hline 340 & $1268-52933-318$ & J083038.23+285852.6 & $1.089 \pm 0.044$ & $8.226 \pm 0.041$ & 8.884 & $-0.890 \pm 0.006$ & $-0.953 \pm 0.009$ & $1.624 \pm 0.004$ & 0.755 \\
\hline 341 & 1269- 52937- 177 & J084219.08+300703.7 & $1.046 \pm 0.039$ & $8.280 \pm 0.038$ & 8.623 & $-1.113 \pm 0.006$ & $-0.877 \pm 0.008$ & $1.816 \pm 0.004$ & 0.711 \\
\hline 342 & 1274- 52995- 258 & J092125.53+345858.4 & $1.134 \pm 0.060$ & $8.212 \pm 0.048$ & 8.417 & $-1.161 \pm 0.010$ & $-0.737 \pm 0.011$ & $1.870 \pm 0.008$ & 0.669 \\
\hline 343 & $1279-52736-147$ & $\mathrm{~J} 125153.04+493216.7$ & $1.158 \pm 0.056$ & $8.193 \pm 0.048$ & 8.128 & $-1.377 \pm 0.012$ & $-0.871 \pm 0.009$ & $2.148 \pm 0.007$ & 0.733 \\
\hline 344 & $1283-52762-315$ & $\mathrm{~J} 132751.58+480805.2$ & $1.080 \pm 0.048$ & $8.250 \pm 0.045$ & 8.572 & $-1.122 \pm 0.009$ & $-0.829 \pm 0.008$ & $1.850 \pm 0.006$ & 0.724 \\
\hline 345 & $1286-52725-150$ & $\mathrm{~J} 141020.62+460501.6$ & $1.210 \pm 0.045$ & $8.133 \pm 0.032$ & 8.507 & $-1.091 \pm 0.008$ & $-0.740 \pm 0.007$ & $1.816 \pm 0.006$ & 0.681 \\
\hline 346 & 1286- 52725- 579 & $\mathrm{~J} 141121.91+472849.4$ & $1.124 \pm 0.052$ & $8.232 \pm 0.042$ & 8.331 & $-1.190 \pm 0.010$ & $-0.741 \pm 0.008$ & $1.905 \pm 0.007$ & 0.666 \\
\hline 347 & $1301-52976-356$ & J090934.68+085837.5 & $1.041 \pm 0.054$ & $8.272 \pm 0.043$ & 8.671 & $-0.888 \pm 0.004$ & $-0.681 \pm 0.005$ & $1.416 \pm 0.003$ & 0.535 \\
\hline 348 & 1303-53050- 66 & J093623.28+090001.0 & $1.166 \pm 0.056$ & $8.201 \pm 0.049$ & 8.701 & $-1.211 \pm 0.015$ & $-0.979 \pm 0.017$ & $2.066 \pm 0.013$ & 0.822 \\
\hline 349 & 1304- 52993- 245 & J093317.21+085425.9 & $1.153 \pm 0.074$ & $8.153 \pm 0.052$ & 8.607 & $-0.909 \pm 0.007$ & $-0.665 \pm 0.006$ & $1.484 \pm 0.004$ & 0.561 \\
\hline 350 & 1306- 52996- 292 & J094756.86+091132.2 & $1.403 \pm 0.032$ & $7.930 \pm 0.023$ & 7.987 & $-1.735 \pm 0.018$ & $-1.087 \pm 0.010$ & $2.549 \pm 0.015$ & 0.826 \\
\hline 351 & $1310-53033-508$ & $\mathrm{~J} 113734.87+572406.1$ & $1.107 \pm 0.047$ & $8.252 \pm 0.040$ & 8.132 & $-1.319 \pm 0.013$ & $-0.787 \pm 0.008$ & $2.065 \pm 0.010$ & 0.704 \\
\hline 352 & 1316- 52790- 581 & $\mathrm{~J} 123801.22+580114.5$ & $1.367 \pm 0.024$ & $8.057 \pm 0.022$ & 8.367 & $-1.575 \pm 0.012$ & $-1.146 \pm 0.014$ & $2.530 \pm 0.009$ & 0.885 \\
\hline 353 & 1324- 53088- 524 & $\mathrm{~J} 140721.21+553807.8$ & $1.001 \pm 0.057$ & $8.387 \pm 0.058$ & 8.590 & $-1.141 \pm 0.009$ & $-0.817 \pm 0.016$ & $1.861 \pm 0.007$ & 0.683 \\
\hline 354 & $1325-52762-519$ & $\mathrm{~J} 141431.20+543056.1$ & $1.260 \pm 0.059$ & $8.091 \pm 0.043$ & 8.256 & $-1.406 \pm 0.027$ & $-1.020 \pm 0.026$ & $2.234 \pm 0.025$ & 0.799 \\
\hline 355 & 1326- 52764- 377 & $\mathrm{~J} 142344.91+535924.7$ & $1.308 \pm 0.067$ & $8.029 \pm 0.045$ & 8.092 & $-1.416 \pm 0.019$ & $-0.835 \pm 0.012$ & $2.192 \pm 0.016$ & 0.755 \\
\hline 356 & 1331- 52766- 258 & $\mathrm{~J} 152115.67+465840.8$ & $1.290 \pm 0.090$ & $7.948 \pm 0.054$ & 8.696 & $-0.903 \pm 0.010$ & $-0.727 \pm 0.011$ & $1.447 \pm 0.008$ & 0.598 \\
\hline 357 & 1331- 52766- 554 & $\mathrm{~J} 152841.69+475445.7$ & $1.247 \pm 0.032$ & $8.075 \pm 0.028$ & 8.362 & $-1.445 \pm 0.012$ & $-1.063 \pm 0.011$ & $2.262 \pm 0.009$ & 0.822 \\
\hline 358 & 1332- 52781- 27 & $\mathrm{~J} 154120.02+453619.0$ & $1.016 \pm 0.051$ & $8.345 \pm 0.053$ & 8.846 & $-0.956 \pm 0.009$ & $-0.915 \pm 0.011$ & $1.713 \pm 0.007$ & 0.756 \\
\hline 359 & 1332- 52781- 602 & $\mathrm{~J} 153821.29+465103.6$ & $1.492 \pm 0.137$ & $7.870 \pm 0.066$ & 8.242 & $-1.197 \pm 0.019$ & $-0.734 \pm 0.018$ & $1.867 \pm 0.016$ & 0.633 \\
\hline 360 & $1333-52782-172$ & $\mathrm{~J} 154544.52+441551.8$ & $1.281 \pm 0.048$ & $8.075 \pm 0.033$ & 8.107 & $-1.462 \pm 0.014$ & $-0.901 \pm 0.010$ & $2.239 \pm 0.011$ & 0.727 \\
\hline 361 & $1335-52824-291$ & $\mathrm{~J} 155944.57+403325.2$ & $1.195 \pm 0.053$ & $8.167 \pm 0.045$ & 8.310 & $-1.369 \pm 0.021$ & $-0.995 \pm 0.020$ & $2.221 \pm 0.019$ & 0.820 \\
\hline 362 & $1338-52765-329$ & $\mathrm{~J} 162350.72+371003.7$ & $1.106 \pm 0.036$ & $8.290 \pm 0.034$ & 8.509 & $-1.329 \pm 0.010$ & $-0.965 \pm 0.011$ & $2.152 \pm 0.008$ & 0.756 \\
\hline 363 & 1345- 52814- 364 & $\mathrm{~J} 134251.19+441343.3$ & $1.068 \pm 0.065$ & $8.268 \pm 0.064$ & 8.171 & $-1.335 \pm 0.014$ & $-0.882 \pm 0.011$ & $2.100 \pm 0.011$ & 0.770 \\
\hline 364 & 1349- 52797- 175 & $\mathrm{~J} 143248.38+395917.8$ & $1.224 \pm 0.021$ & $8.148 \pm 0.019$ & 8.434 & $-1.393 \pm 0.009$ & $-0.993 \pm 0.008$ & $2.245 \pm 0.007$ & 0.807 \\
\hline 365 & 1349- 52797- 429 & $\mathrm{~J} 143036.00+402413.6$ & $1.193 \pm 0.065$ & $8.186 \pm 0.051$ & 8.187 & $-1.396 \pm 0.018$ & $-0.907 \pm 0.013$ & $2.206 \pm 0.015$ & 0.743 \\
\hline 366 & 1355- 52823- 98 & $\mathrm{~J} 153536.14+332843.3$ & $0.984 \pm 0.052$ & $8.441 \pm 0.059$ & 8.531 & $-1.209 \pm 0.008$ & $-0.850 \pm 0.008$ & $1.978 \pm 0.004$ & 0.700 \\
\hline 367 & 1356- 53033- 248 & $\mathrm{~J} 100052.15+383056.8$ & $1.016 \pm 0.051$ & $8.347 \pm 0.052$ & 8.573 & $-0.988 \pm 0.007$ & $-0.783 \pm 0.009$ & $1.699 \pm 0.005$ & 0.690 \\
\hline 368 & 1357- 53034- 245 & $\mathrm{~J} 101207.44+393132.1$ & $1.080 \pm 0.071$ & $8.286 \pm 0.062$ & 8.489 & $-1.085 \pm 0.035$ & $-0.767 \pm 0.010$ & $1.820 \pm 0.034$ & 0.692 \\
\hline 369 & $1357-53034-540$ & $\mathrm{~J} 101541.16+412050.2$ & $1.037 \pm 0.042$ & $8.357 \pm 0.044$ & 8.585 & $-1.209 \pm 0.009$ & $-0.960 \pm 0.009$ & $2.015 \pm 0.006$ & 0.760 \\
\hline
\end{tabular}


Table 1. continued.

\begin{tabular}{|c|c|c|c|c|c|c|c|c|c|}
\hline (1) & (2) & (3) & (4) & (5) & (6) & (7) & (8) & (9) & (10) \\
\hline Num & Plate-MJD-FiberID & IAU designations & $t_{3}\left(10^{4} \mathrm{~K}\right)$ & $12+\log (\mathrm{O} / \mathrm{H})_{T_{\mathrm{e}}}$ & $(\mathrm{O} / \mathrm{H})_{\text {Bay }}$ & $\mathrm{N} 2$ & S2 & O3N2 & $P$ \\
\hline 370 & 1357- 53034- 579 & $\mathrm{~J} 101803.24+410621.2$ & $1.407 \pm 0.036$ & $7.979 \pm 0.026$ & 8.649 & $-1.440 \pm 0.023$ & $-1.186 \pm 0.032$ & $2.340 \pm 0.021$ & 0.872 \\
\hline 371 & 1363- 53053- 411 & $\mathrm{~J} 110251.98+433827.2$ & $1.135 \pm 0.045$ & $8.206 \pm 0.036$ & 8.077 & $-1.390 \pm 0.012$ & $-0.817 \pm 0.008$ & $2.117 \pm 0.009$ & 0.696 \\
\hline 372 & 1363- 53053- 599 & $\mathrm{~J} 110744.09+443825.8$ & $1.074 \pm 0.058$ & $8.286 \pm 0.053$ & 8.423 & $-1.221 \pm 0.010$ & $-0.805 \pm 0.009$ & $1.948 \pm 0.006$ & 0.688 \\
\hline 373 & $1365-53062-381$ & $\mathrm{~J} 111727.26+450043.2$ & $1.349 \pm 0.031$ & $8.072 \pm 0.029$ & 8.182 & $-1.518 \pm 0.024$ & $-1.322 \pm 0.030$ & $2.497 \pm 0.022$ & 0.919 \\
\hline 374 & $1368-53084-334$ & $\mathrm{~J} 114424.02+443630.2$ & $1.102 \pm 0.055$ & $8.198 \pm 0.046$ & 8.505 & $-1.167 \pm 0.010$ & $-0.773 \pm 0.007$ & $1.827 \pm 0.007$ & 0.673 \\
\hline 375 & $1372-53062-517$ & $\mathrm{~J} 123915.79+444800.7$ & $0.964 \pm 0.045$ & $8.368 \pm 0.049$ & 8.622 & $-0.969 \pm 0.006$ & $-0.776 \pm 0.006$ & $1.607 \pm 0.004$ & 0.666 \\
\hline 376 & 1382- 53115- 231 & $\mathrm{~J} 143532.88+364630.7$ & $1.295 \pm 0.087$ & $8.010 \pm 0.053$ & 8.243 & $-1.183 \pm 0.015$ & $-0.755 \pm 0.010$ & $1.825 \pm 0.013$ & 0.629 \\
\hline 377 & 1388- 53119- 39 & $\mathrm{~J} 153656.45+312248.0$ & $1.088 \pm 0.052$ & $8.260 \pm 0.045$ & 8.589 & $-1.110 \pm 0.007$ & $-0.829 \pm 0.007$ & $1.811 \pm 0.004$ & 0.666 \\
\hline 378 & 1391- 52817- 231 & $\mathrm{~J} 155338.85+280257.8$ & $1.074 \pm 0.068$ & $8.222 \pm 0.054$ & 8.780 & $-0.708 \pm 0.005$ & $-0.696 \pm 0.006$ & $1.241 \pm 0.003$ & 0.547 \\
\hline 379 & 1394- 53108- 259 & $\mathrm{~J} 141240.42+424213.3$ & $1.210 \pm 0.090$ & $8.156 \pm 0.062$ & 8.146 & $-1.290 \pm 0.013$ & $-0.695 \pm 0.010$ & $1.978 \pm 0.011$ & 0.613 \\
\hline 380 & 1395- 52825- 242 & $\mathrm{~J} 142405.74+421646.2$ & $1.353 \pm 0.018$ & $8.059 \pm 0.019$ & 8.786 & $-1.330 \pm 0.009$ & $-1.180 \pm 0.012$ & $2.276 \pm 0.007$ & 0.889 \\
\hline 381 & 1398- 53146- 573 & $\mathrm{~J} 150321.55+401652.6$ & $1.494 \pm 0.138$ & $7.863 \pm 0.069$ & 8.134 & $-1.280 \pm 0.021$ & $-0.731 \pm 0.017$ & $1.948 \pm 0.017$ & 0.636 \\
\hline 382 & 1399- 53172- 204 & $\mathrm{~J} 151242.96+372535.0$ & $1.067 \pm 0.068$ & $8.321 \pm 0.068$ & 8.550 & $-1.248 \pm 0.018$ & $-0.956 \pm 0.017$ & $2.070 \pm 0.015$ & 0.776 \\
\hline 383 & 1401- 53144- 397 & $\mathrm{~J} 152821.98+362409.3$ & $1.069 \pm 0.042$ & $8.323 \pm 0.043$ & 8.543 & $-1.290 \pm 0.011$ & $-0.968 \pm 0.010$ & $2.126 \pm 0.008$ & 0.791 \\
\hline 384 & $1405-52826-395$ & $\mathrm{~J} 160135.95+311353.7$ & $1.021 \pm 0.061$ & $8.335 \pm 0.063$ & 8.585 & $-1.135 \pm 0.010$ & $-0.842 \pm 0.010$ & $1.861 \pm 0.007$ & 0.715 \\
\hline 385 & 1409- 52824- 461 & $\mathrm{~J} 163305.62+260026.6$ & $1.920 \pm 0.219$ & $7.595 \pm 0.068$ & 8.046 & $-1.539 \pm 0.045$ & $-0.805 \pm 0.021$ & $2.183 \pm 0.041$ & 0.617 \\
\hline 386 & 1418- 53142- 442 & $\mathrm{~J} 160055.10+344551.8$ & $1.155 \pm 0.055$ & $8.138 \pm 0.042$ & 8.615 & $-1.051 \pm 0.008$ & $-0.794 \pm 0.008$ & $1.712 \pm 0.006$ & 0.672 \\
\hline 387 & 1425- 52913- 518 & $\mathrm{~J} 170450.35+204718.9$ & $1.052 \pm 0.053$ & $8.359 \pm 0.053$ & 8.500 & $-1.247 \pm 0.011$ & $-0.886 \pm 0.010$ & $2.056 \pm 0.009$ & 0.738 \\
\hline 388 & $1428-52998-568$ & J102847.64+394941.5 & $0.923 \pm 0.047$ & $8.515 \pm 0.055$ & 8.623 & $-1.071 \pm 0.007$ & $-0.759 \pm 0.006$ & $1.695 \pm 0.004$ & 0.558 \\
\hline 389 & $1430-53002-389$ & $\mathrm{~J} 102909.31+394426.1$ & $0.955 \pm 0.046$ & $8.401 \pm 0.052$ & 8.602 & $-1.108 \pm 0.007$ & $-0.815 \pm 0.007$ & $1.780 \pm 0.005$ & 0.684 \\
\hline 390 & $1442-53050-396$ & $\mathrm{~J} 112546.80+470000.3$ & $0.865 \pm 0.044$ & $8.557 \pm 0.059$ & 8.688 & $-0.963 \pm 0.005$ & $-0.846 \pm 0.006$ & $1.627 \pm 0.003$ & 0.665 \\
\hline 391 & $1445-53062-429$ & $\mathrm{~J} 114138.04+423437.2$ & $1.299 \pm 0.060$ & $8.024 \pm 0.039$ & 8.065 & $-1.365 \pm 0.015$ & $-0.837 \pm 0.011$ & $2.093 \pm 0.013$ & 0.709 \\
\hline 392 & 1454- 53090- 204 & $\mathrm{~J} 123506.50+413736.4$ & $0.975 \pm 0.058$ & $8.358 \pm 0.062$ & 8.735 & $-0.902 \pm 0.007$ & $-0.800 \pm 0.009$ & $1.556 \pm 0.005$ & 0.679 \\
\hline 393 & 1455- 53089- 287 & $\mathrm{~J} 123803.77+461820.1$ & $1.243 \pm 0.026$ & $8.153 \pm 0.022$ & 8.662 & $-1.262 \pm 0.008$ & $-0.979 \pm 0.008$ & $2.135 \pm 0.005$ & 0.800 \\
\hline 394 & 1457- 53116- 381 & $\mathrm{~J} 125251.63+480508.1$ & $1.059 \pm 0.068$ & $8.340 \pm 0.064$ & 8.225 & $-1.307 \pm 0.016$ & $-0.825 \pm 0.010$ & $2.063 \pm 0.013$ & 0.680 \\
\hline 395 & $1462-53112-184$ & $\mathrm{~J} 132032.04+405901.6$ & $1.020 \pm 0.064$ & $8.326 \pm 0.063$ & 8.708 & $-1.041 \pm 0.008$ & $-0.897 \pm 0.009$ & $1.717 \pm 0.006$ & 0.671 \\
\hline 396 & 1464- 53091- 232 & $\mathrm{~J} 133037.68+401953.0$ & $1.016 \pm 0.041$ & $8.356 \pm 0.042$ & 8.156 & $-1.304 \pm 0.008$ & $-0.821 \pm 0.008$ & $2.037 \pm 0.006$ & 0.706 \\
\hline 397 & 1464- 53091- 442 & $\mathrm{~J} 133346.30+415213.0$ & $1.138 \pm 0.064$ & $8.226 \pm 0.050$ & 8.460 & $-1.131 \pm 0.011$ & $-0.727 \pm 0.008$ & $1.828 \pm 0.008$ & 0.633 \\
\hline 398 & 1466- 53083- 92 & $\mathrm{~J} 135403.26+442616.4$ & $1.065 \pm 0.063$ & $8.296 \pm 0.057$ & 8.588 & $-1.090 \pm 0.008$ & $-0.797 \pm 0.008$ & $1.780 \pm 0.005$ & 0.645 \\
\hline 399 & 1467- 53115- 32 & $\mathrm{~J} 141022.27+441455.6$ & $1.079 \pm 0.066$ & $8.244 \pm 0.059$ & 8.296 & $-1.183 \pm 0.009$ & $-0.752 \pm 0.008$ & $1.851 \pm 0.007$ & 0.660 \\
\hline 400 & 1467- 53115- 579 & $\mathrm{~J} 141007.10+450817.5$ & $1.057 \pm 0.047$ & $8.274 \pm 0.038$ & 8.597 & $-0.975 \pm 0.006$ & $-0.674 \pm 0.006$ & $1.563 \pm 0.003$ & 0.570 \\
\hline 401 & 1474- 52933- 51 & J220200.67-011208.6 & $1.302 \pm 0.060$ & $8.041 \pm 0.043$ & 8.056 & $-1.621 \pm 0.029$ & $-0.955 \pm 0.016$ & $2.436 \pm 0.027$ & 0.795 \\
\hline 402 & $1476-52964-530$ & $\mathrm{~J} 221549.25+010938.8$ & $1.077 \pm 0.052$ & $8.264 \pm 0.049$ & 8.091 & $-1.347 \pm 0.013$ & $-0.803 \pm 0.009$ & $2.098 \pm 0.011$ & 0.742 \\
\hline 403 & 1487- 52964- 510 & $\mathrm{~J} 234249.56+002440.3$ & $1.309 \pm 0.049$ & $8.011 \pm 0.034$ & 8.025 & $-1.519 \pm 0.032$ & $-0.939 \pm 0.011$ & $2.300 \pm 0.030$ & 0.783 \\
\hline 404 & 1491- 52996- 350 & $\mathrm{~J} 000938.35+002535.7$ & $1.546 \pm 0.147$ & $7.801 \pm 0.070$ & 8.093 & $-1.416 \pm 0.044$ & $-0.835 \pm 0.020$ & $2.156 \pm 0.041$ & 0.755 \\
\hline 405 & 1492- 52932- 10 & J002425.03-010359.0 & $1.498 \pm 0.046$ & $7.895 \pm 0.032$ & 7.998 & $-1.808 \pm 0.028$ & $-1.180 \pm 0.016$ & $2.705 \pm 0.025$ & 0.884 \\
\hline 406 & 1495- 52944- 627 & J004829.64+003710.5 & $1.369 \pm 0.049$ & $7.983 \pm 0.031$ & 8.082 & $-1.514 \pm 0.019$ & $-0.892 \pm 0.009$ & $2.276 \pm 0.014$ & 0.724 \\
\hline 407 & $1522-52932-40$ & J212657.96-003227.9 & $1.713 \pm 0.152$ & $7.744 \pm 0.062$ & 8.124 & $-1.562 \pm 0.049$ & $-0.902 \pm 0.031$ & $2.359 \pm 0.047$ & 0.760 \\
\hline 408 & $1562-53052-557$ & J025754.79+002726.6 & $1.250 \pm 0.043$ & $8.179 \pm 0.036$ & 8.116 & $-1.581 \pm 0.023$ & $-1.066 \pm 0.017$ & $2.528 \pm 0.021$ & 0.855 \\
\hline 409 & $1570-53149-146$ & $\mathrm{~J} 164202.38+210345.7$ & $1.067 \pm 0.045$ & $8.219 \pm 0.039$ & 8.816 & $-0.801 \pm 0.005$ & $-0.798 \pm 0.005$ & $1.420 \pm 0.002$ & 0.654 \\
\hline 410 & $1571-53174-155$ & J163527.94+222518.8 & $0.959 \pm 0.053$ & $8.413 \pm 0.060$ & 8.557 & $-1.200 \pm 0.007$ & $-0.821 \pm 0.007$ & $1.893 \pm 0.005$ & 0.690 \\
\hline 411 & $1580-53145-418$ & $\mathrm{~J} 154613.87+330423.1$ & $1.087 \pm 0.048$ & $8.241 \pm 0.044$ & 8.592 & $-1.090 \pm 0.008$ & $-0.841 \pm 0.008$ & $1.814 \pm 0.005$ & 0.718 \\
\hline 412 & $1580-53145-419$ & $\mathrm{~J} 154659.02+325632.2$ & $1.277 \pm 0.034$ & $8.110 \pm 0.030$ & 8.137 & $-1.543 \pm 0.030$ & $-1.196 \pm 0.016$ & $2.463 \pm 0.028$ & 0.879 \\
\hline 413 & 1584- 52943- 372 & J080824.94+230840.9 & $0.981 \pm 0.048$ & $8.413 \pm 0.048$ & 8.780 & $-0.846 \pm 0.006$ & $-0.826 \pm 0.007$ & $1.542 \pm 0.004$ & 0.654 \\
\hline 414 & 1584- 52943- 486 & J080911.47+224758.9 & $1.113 \pm 0.069$ & $8.238 \pm 0.050$ & 8.575 & $-0.973 \pm 0.007$ & $-0.635 \pm 0.010$ & $1.555 \pm 0.005$ & 0.527 \\
\hline 415 & $1590-52974-628$ & $\mathrm{~J} 090405.90+313045.0$ & $1.332 \pm 0.082$ & $7.921 \pm 0.047$ & 8.613 & $-1.022 \pm 0.008$ & $-0.725 \pm 0.009$ & $1.614 \pm 0.005$ & 0.635 \\
\hline 416 & 1595- 52999- 532 & J095115.96+364031.0 & $1.041 \pm 0.061$ & $8.267 \pm 0.053$ & 8.623 & $-0.914 \pm 0.006$ & $-0.676 \pm 0.006$ & $1.469 \pm 0.004$ & 0.561 \\
\hline 417 & 1597- 52999- 50 & $\mathrm{~J} 101828.39+095412.2$ & $1.021 \pm 0.063$ & $8.270 \pm 0.060$ & 8.663 & $-0.994 \pm 0.006$ & $-0.768 \pm 0.006$ & $1.588 \pm 0.004$ & 0.636 \\
\hline 418 & 1597- 52999- 168 & $\mathrm{~J} 101546.87+101234.5$ & $1.131 \pm 0.076$ & $8.230 \pm 0.064$ & 8.189 & $-1.304 \pm 0.020$ & $-0.806 \pm 0.012$ & $2.062 \pm 0.017$ & 0.711 \\
\hline 419 & 1597- 52999- 251 & $\mathrm{~J} 101139.17+101042.2$ & $1.059 \pm 0.053$ & $8.325 \pm 0.051$ & 8.120 & $-1.366 \pm 0.013$ & $-0.811 \pm 0.009$ & $2.123 \pm 0.010$ & 0.703 \\
\hline 420 & 1597- 52999- 358 & $\mathrm{~J} 100950.23+110439.3$ & $1.024 \pm 0.042$ & $8.301 \pm 0.042$ & 8.598 & $-1.153 \pm 0.007$ & $-0.830 \pm 0.007$ & $1.851 \pm 0.005$ & 0.717 \\
\hline 421 & $1601-53115-168$ & $\mathrm{~J} 104829.23+111520.1$ & $1.224 \pm 0.027$ & $8.161 \pm 0.024$ & 8.161 & $-1.421 \pm 0.010$ & $-0.909 \pm 0.012$ & $2.295 \pm 0.008$ & 0.815 \\
\hline 422 & $1601-53115-526$ & $\mathrm{~J} 104819.42+123745.8$ & $1.324 \pm 0.103$ & $7.944 \pm 0.059$ & 8.627 & $-1.048 \pm 0.015$ & $-0.717 \pm 0.017$ & $1.623 \pm 0.012$ & 0.598 \\
\hline 423 & 1607- 53083- 205 & $\mathrm{~J} 113530.91+111717.8$ & $1.104 \pm 0.040$ & $8.245 \pm 0.036$ & 8.112 & $-1.327 \pm 0.011$ & $-0.819 \pm 0.008$ & $2.077 \pm 0.009$ & 0.721 \\
\hline
\end{tabular}


Table 1. continued.

\begin{tabular}{|c|c|c|c|c|c|c|c|c|c|}
\hline (1) & (2) & (3) & (4) & (5) & (6) & (7) & (8) & (9) & (10) \\
\hline Num & Plate-MJD-FiberID & IAU designations & $t_{3}\left(10^{4} \mathrm{~K}\right)$ & $12+\log (\mathrm{O} / \mathrm{H})_{T_{\mathrm{e}}}$ & $(\mathrm{O} / \mathrm{H})_{\text {Bay }}$ & N2 & S2 & O3N2 & $P$ \\
\hline 424 & $1609-53142-238$ & $\mathrm{~J} 114837.49+121407.8$ & $1.170 \pm 0.045$ & $8.142 \pm 0.036$ & 8.041 & $-1.360 \pm 0.010$ & $-0.861 \pm 0.008$ & $2.096 \pm 0.008$ & 0.738 \\
\hline 425 & 1615- 53166- 206 & $\mathrm{~J} 122900.39+112302.0$ & $1.105 \pm 0.069$ & $8.247 \pm 0.061$ & 8.536 & $-1.148 \pm 0.011$ & $-0.789 \pm 0.010$ & $1.879 \pm 0.008$ & 0.693 \\
\hline 426 & 1616- 53169- 205 & $\mathrm{~J} 123534.25+111926.0$ & $1.151 \pm 0.039$ & $8.247 \pm 0.034$ & 8.118 & $-1.410 \pm 0.014$ & $-0.875 \pm 0.009$ & $2.237 \pm 0.011$ & 0.745 \\
\hline 427 & $1620-53137-470$ & $\mathrm{~J} 114228.75+075709.3$ & $0.991 \pm 0.059$ & $8.403 \pm 0.062$ & 8.498 & $-1.232 \pm 0.012$ & $-0.844 \pm 0.015$ & $1.974 \pm 0.009$ & 0.706 \\
\hline 428 & $1623-53089-493$ & $\mathrm{~J} 120344.83+072903.8$ & $1.234 \pm 0.089$ & $8.071 \pm 0.062$ & 8.099 & $-1.268 \pm 0.015$ & $-0.694 \pm 0.011$ & $1.918 \pm 0.012$ & 0.638 \\
\hline 429 & 1632- 52996- 135 & J034254.29-005520.2 & $1.124 \pm 0.045$ & $8.229 \pm 0.039$ & 8.190 & $-1.341 \pm 0.022$ & $-0.910 \pm 0.011$ & $2.132 \pm 0.021$ & 0.762 \\
\hline 430 & 1644- 53144- 564 & $\mathrm{~J} 142805.52+362710.4$ & $1.418 \pm 0.022$ & $7.941 \pm 0.017$ & 8.065 & $-1.502 \pm 0.009$ & $-1.029 \pm 0.008$ & $2.333 \pm 0.006$ & 0.816 \\
\hline 431 & 1648- 53171- 21 & $\mathrm{~J} 150950.07+314639.7$ & $1.238 \pm 0.076$ & $8.084 \pm 0.057$ & 8.042 & $-1.439 \pm 0.018$ & $-0.868 \pm 0.013$ & $2.191 \pm 0.015$ & 0.739 \\
\hline 432 & $1650-53174-305$ & $\mathrm{~J} 151634.77+300653.6$ & $0.988 \pm 0.044$ & $8.387 \pm 0.047$ & 8.498 & $-1.098 \pm 0.007$ & $-0.832 \pm 0.006$ & $1.812 \pm 0.004$ & 0.694 \\
\hline 433 & $1682-53173-3$ & $\mathrm{~J} 160810.37+352809.1$ & $1.800 \pm 0.039$ & $7.745 \pm 0.031$ & 7.879 & $-2.528 \pm 0.054$ & $-1.833 \pm 0.032$ & $3.529 \pm 0.047$ & 0.980 \\
\hline 434 & 1689- 53177- 521 & $\mathrm{~J} 171108.98+234547.1$ & $1.279 \pm 0.049$ & $8.075 \pm 0.034$ & 8.094 & $-1.515 \pm 0.017$ & $-0.897 \pm 0.010$ & $2.312 \pm 0.015$ & 0.756 \\
\hline 435 & $1697-53142-48$ & $\mathrm{~J} 131644.79+105733.1$ & $1.123 \pm 0.033$ & $8.259 \pm 0.033$ & 8.188 & $-1.425 \pm 0.018$ & $-0.959 \pm 0.009$ & $2.275 \pm 0.016$ & 0.805 \\
\hline 436 & $1702-53144-62$ & $\mathrm{~J} 135832.26+104718.9$ & $1.202 \pm 0.025$ & $8.222 \pm 0.026$ & 8.794 & $-1.466 \pm 0.011$ & $-1.199 \pm 0.011$ & $2.414 \pm 0.008$ & 0.867 \\
\hline 437 & 1704- 53178- 345 & $\mathrm{~J} 140158.15+134830.2$ & $1.177 \pm 0.069$ & $8.163 \pm 0.049$ & 8.277 & $-1.180 \pm 0.010$ & $-0.716 \pm 0.010$ & $1.841 \pm 0.007$ & 0.613 \\
\hline 438 & 1704- 53178- 478 & $\mathrm{~J} 140555.23+140528.3$ & $1.185 \pm 0.077$ & $8.149 \pm 0.055$ & 8.159 & $-1.248 \pm 0.014$ & $-0.741 \pm 0.010$ & $1.922 \pm 0.012$ & 0.634 \\
\hline 439 & $1734-53034-490$ & $\mathrm{~J} 073149.49+404513.3$ & $1.224 \pm 0.031$ & $8.145 \pm 0.028$ & 8.106 & $-1.549 \pm 0.012$ & $-1.013 \pm 0.008$ & $2.409 \pm 0.009$ & 0.820 \\
\hline 440 & 1744- 53055- 450 & $\mathrm{~J} 100307.77+130326.2$ & $1.015 \pm 0.061$ & $8.339 \pm 0.058$ & 8.614 & $-0.991 \pm 0.007$ & $-0.742 \pm 0.007$ & $1.630 \pm 0.004$ & 0.618 \\
\hline 441 & $1745-53061-463$ & $\mathrm{~J} 101157.09+130822.2$ & $1.437 \pm 0.018$ & $8.000 \pm 0.019$ & 8.361 & $-1.644 \pm 0.013$ & $-1.349 \pm 0.013$ & $2.622 \pm 0.011$ & 0.918 \\
\hline 442 & $1747-53075-182$ & $\mathrm{~J} 102744.14+130934.2$ & $1.043 \pm 0.054$ & $8.307 \pm 0.053$ & 8.589 & $-1.039 \pm 0.007$ & $-0.817 \pm 0.008$ & $1.772 \pm 0.005$ & 0.720 \\
\hline 443 & 1758- 53084- 338 & $\mathrm{~J} 082520.11+082723.0$ & $1.122 \pm 0.042$ & $8.265 \pm 0.040$ & 8.379 & $-1.423 \pm 0.014$ & $-1.016 \pm 0.016$ & $2.287 \pm 0.011$ & 0.818 \\
\hline 444 & $1821-53167-496$ & $\mathrm{~J} 154453.28+062452.9$ & $1.314 \pm 0.089$ & $7.985 \pm 0.056$ & 8.034 & $-1.337 \pm 0.017$ & $-0.770 \pm 0.013$ & $2.016 \pm 0.015$ & 0.681 \\
\hline 445 & $282-51630-546$ & $\mathrm{~J} 113703.79+002817.4$ & $1.156 \pm 0.034$ & $8.248 \pm 0.031$ & 8.186 & $-1.443 \pm 0.018$ & $-0.916 \pm 0.010$ & $2.333 \pm 0.016$ & 0.822 \\
\hline 446 & 296- 51665- 411 & J131937.25+005043.8 & $1.062 \pm 0.037$ & $8.346 \pm 0.038$ & 8.485 & $-1.364 \pm 0.012$ & $-0.995 \pm 0.010$ & $2.199 \pm 0.008$ & 0.770 \\
\hline 447 & 297- 51663- 446 & $\mathrm{~J} 132654.62+011346.5$ & $1.209 \pm 0.028$ & $8.135 \pm 0.025$ & 8.103 & $-1.445 \pm 0.012$ & $-0.977 \pm 0.012$ & $2.286 \pm 0.011$ & 0.828 \\
\hline 448 & $301-51641-525$ & $\mathrm{~J} 140018.94+010453.7$ & $1.232 \pm 0.025$ & $8.124 \pm 0.021$ & 8.536 & $-1.303 \pm 0.008$ & $-0.955 \pm 0.008$ & $2.135 \pm 0.006$ & 0.799 \\
\hline 449 & 304- 51609- 583 & $\mathrm{~J} 142200.19+010213.2$ & $1.011 \pm 0.053$ & $8.359 \pm 0.052$ & 8.553 & $-1.189 \pm 0.008$ & $-0.800 \pm 0.011$ & $1.893 \pm 0.006$ & 0.677 \\
\hline 450 & 309- 51666- 282 & $\mathrm{~J} 145146.99-005643.8$ & $1.162 \pm 0.026$ & $8.188 \pm 0.023$ & 8.067 & $-1.545 \pm 0.013$ & $-0.976 \pm 0.008$ & $2.346 \pm 0.011$ & 0.773 \\
\hline 451 & 348- 51696- 339 & $\mathrm{~J} 163107.20+005324.7$ & $1.168 \pm 0.061$ & $8.177 \pm 0.047$ & 8.088 & $-1.384 \pm 0.011$ & $-0.828 \pm 0.008$ & $2.116 \pm 0.009$ & 0.692 \\
\hline 452 & $351-51695-217$ & $\mathrm{~J} 170201.44+604746.3$ & $1.034 \pm 0.041$ & $8.293 \pm 0.035$ & 8.624 & $-0.961 \pm 0.005$ & $-0.747 \pm 0.005$ & $1.568 \pm 0.003$ & 0.602 \\
\hline 453 & $394-51812-480$ & J005147.30+000939.9 & $1.566 \pm 0.023$ & $7.782 \pm 0.018$ & 7.866 & $-1.880 \pm 0.024$ & $-1.260 \pm 0.010$ & $2.709 \pm 0.022$ & 0.880 \\
\hline 454 & 394- 51876- 472 & $\mathrm{~J} 005147.30+000939.9$ & $1.548 \pm 0.018$ & $7.797 \pm 0.017$ & 7.864 & $-1.873 \pm 0.018$ & $-1.289 \pm 0.009$ & $2.718 \pm 0.016$ & 0.898 \\
\hline 455 & 406- 51817- 490 & J022407.68+003226.1 & $1.085 \pm 0.070$ & $8.263 \pm 0.059$ & 8.125 & $-1.272 \pm 0.011$ & $-0.708 \pm 0.007$ & $1.919 \pm 0.008$ & 0.607 \\
\hline 456 & 406- 51900- 204 & J022312.62-004539.9 & $1.432 \pm 0.125$ & $7.906 \pm 0.063$ & 8.499 & $-1.015 \pm 0.013$ & $-0.630 \pm 0.012$ & $1.597 \pm 0.010$ & 0.550 \\
\hline 457 & 406- $51900-488$ & J022407.68+003226.1 & $1.183 \pm 0.068$ & $8.158 \pm 0.048$ & 8.130 & $-1.281 \pm 0.011$ & $-0.709 \pm 0.008$ & $1.920 \pm 0.008$ & 0.588 \\
\hline 458 & 414- 51869- 459 & $\mathrm{~J} 032724.17+004804.3$ & $1.026 \pm 0.051$ & $8.310 \pm 0.047$ & 8.661 & $-1.086 \pm 0.007$ & $-0.841 \pm 0.009$ & $1.751 \pm 0.005$ & 0.667 \\
\hline 459 & 414- 51869- 524 & $\mathrm{~J} 032750.16+010135.0$ & $1.040 \pm 0.061$ & $8.343 \pm 0.054$ & 8.445 & $-1.131 \pm 0.008$ & $-0.720 \pm 0.007$ & $1.788 \pm 0.006$ & 0.593 \\
\hline 460 & 415- 51879- 284 & $\mathrm{~J} 033031.22-005846.5$ & $1.139 \pm 0.039$ & $8.250 \pm 0.037$ & 8.132 & $-1.518 \pm 0.016$ & $-0.991 \pm 0.009$ & $2.382 \pm 0.014$ & 0.807 \\
\hline 461 & 419- 51812- 362 & J004236.94+160202.7 & $1.059 \pm 0.064$ & $8.319 \pm 0.063$ & 8.821 & $-1.105 \pm 0.012$ & $-1.050 \pm 0.028$ & $1.931 \pm 0.010$ & 0.800 \\
\hline 462 & 419- 51868- 362 & J004236.94+160202.7 & $1.004 \pm 0.059$ & $8.393 \pm 0.066$ & 8.826 & $-1.063 \pm 0.011$ & $-1.107 \pm 0.040$ & $1.894 \pm 0.010$ & 0.809 \\
\hline 463 & $425-51884-635$ & $\mathrm{~J} 013700.31+144157.1$ & $1.028 \pm 0.063$ & $8.301 \pm 0.063$ & 8.052 & $-1.295 \pm 0.009$ & $-0.779 \pm 0.006$ & $2.001 \pm 0.007$ & 0.719 \\
\hline 464 & 437- 51876- 460 & $\mathrm{~J} 080147.11+435302.0$ & $1.094 \pm 0.034$ & $8.271 \pm 0.031$ & 8.584 & $-1.236 \pm 0.008$ & $-0.912 \pm 0.009$ & $2.017 \pm 0.006$ & 0.746 \\
\hline 465 & 483- $51942-474$ & $\mathrm{~J} 090047.45+574255.0$ & $0.985 \pm 0.048$ & $8.409 \pm 0.053$ & 8.767 & $-1.053 \pm 0.006$ & $-0.934 \pm 0.009$ & $1.830 \pm 0.004$ & 0.754 \\
\hline 466 & 483- 51942- 586 & J090139.89+575945.9 & $1.114 \pm 0.045$ & $8.235 \pm 0.037$ & 8.127 & $-1.317 \pm 0.008$ & $-0.813 \pm 0.007$ & $2.048 \pm 0.006$ & 0.697 \\
\hline 467 & 594- 52027- 516 & $\mathrm{~J} 154654.55+030902.1$ & $1.109 \pm 0.034$ & $8.286 \pm 0.032$ & 8.532 & $-1.302 \pm 0.012$ & $-0.968 \pm 0.011$ & $2.149 \pm 0.010$ & 0.788 \\
\hline 468 & 616- 52442- 336 & $\mathrm{~J} 153534.13+545534.3$ & $1.083 \pm 0.054$ & $8.314 \pm 0.047$ & 8.568 & $-1.125 \pm 0.009$ & $-0.832 \pm 0.019$ & $1.913 \pm 0.007$ & 0.726 \\
\hline 469 & $662-52178-466$ & J013258.54-085337.6 & $1.003 \pm 0.041$ & $8.381 \pm 0.042$ & 8.349 & $-1.147 \pm 0.007$ & $-0.700 \pm 0.007$ & $1.844 \pm 0.002$ & 0.656 \\
\hline 470 & $673-52162-73$ & $\mathrm{~J} 223126.30-000456.2$ & $1.038 \pm 0.065$ & $8.328 \pm 0.062$ & 8.575 & $-1.100 \pm 0.008$ & $-0.776 \pm 0.007$ & $1.797 \pm 0.005$ & 0.657 \\
\hline 471 & $675-52590-39$ & $\mathrm{~J} 225059.28+000032.7$ & $1.763 \pm 0.049$ & $7.697 \pm 0.032$ & 7.909 & $-1.639 \pm 0.014$ & $-1.445 \pm 0.034$ & $2.543 \pm 0.007$ & 0.934 \\
\hline 472 & 676- 52178- 192 & $\mathrm{~J} 225059.28+000032.7$ & $1.628 \pm 0.033$ & $7.758 \pm 0.023$ & 7.855 & $-1.687 \pm 0.013$ & $-1.398 \pm 0.023$ & $2.559 \pm 0.011$ & 0.916 \\
\hline 473 & $677-52606-374$ & J225833.74+005630.4 & $1.247 \pm 0.095$ & $8.088 \pm 0.070$ & 8.319 & $-1.290 \pm 0.023$ & $-0.912 \pm 0.023$ & $2.093 \pm 0.020$ & 0.788 \\
\hline 474 & 677- 52606- 533 & $\mathrm{~J} 230210.00+004939.0$ & $1.731 \pm 0.029$ & $7.655 \pm 0.022$ & 7.835 & $-2.212 \pm 0.030$ & $-1.456 \pm 0.015$ & $3.039 \pm 0.027$ & 0.910 \\
\hline 475 & 678- 52884- 446 & $\mathrm{~J} 230703.74+011311.2$ & $1.002 \pm 0.049$ & $8.334 \pm 0.047$ & 8.819 & $-0.833 \pm 0.005$ & $-0.819 \pm 0.006$ & $1.467 \pm 0.003$ & 0.645 \\
\hline 476 & 681- 52199- 201 & $\mathrm{~J} 232936.56-011057.0$ & $1.202 \pm 0.027$ & $8.176 \pm 0.025$ & 8.225 & $-1.394 \pm 0.010$ & $-0.937 \pm 0.007$ & $2.255 \pm 0.008$ & 0.813 \\
\hline 477 & $681-52199-595$ & J $233435.40+002714.4$ & $1.315 \pm 0.102$ & $8.038 \pm 0.061$ & 8.189 & $-1.269 \pm 0.039$ & $-0.700 \pm 0.014$ & $1.927 \pm 0.035$ & 0.597 \\
\hline
\end{tabular}


Table 1. continued.

\begin{tabular}{|c|c|c|c|c|c|c|c|c|c|}
\hline (1) & (2) & (3) & (4) & (5) & (6) & (7) & (8) & (9) & (10) \\
\hline Num & Plate-MJD-FiberID & IAU designations & $t_{3}\left(10^{4} \mathrm{~K}\right)$ & $12+\log (\mathrm{O} / \mathrm{H})_{T \mathrm{e}}$ & $(\mathrm{O} / \mathrm{H})_{\text {Bay }}$ & N2 & S2 & O3N2 & $P$ \\
\hline 478 & $682-52525-63$ & J234209.77-002451.1 & $1.282 \pm 0.065$ & $8.103 \pm 0.047$ & 8.122 & $-1.593 \pm 0.027$ & $-0.997 \pm 0.035$ & $2.448 \pm 0.025$ & 0.790 \\
\hline 479 & $682-52525-172$ & J233751.94-001000.4 & $1.809 \pm 0.033$ & $7.738 \pm 0.020$ & 8.048 & $-1.804 \pm 0.017$ & $-1.331 \pm 0.012$ & $2.748 \pm 0.014$ & 0.899 \\
\hline 480 & 683- 52524- 279 & $\mathrm{~J} 234209.77-002451.1$ & $1.140 \pm 0.062$ & $8.251 \pm 0.056$ & 8.139 & $-1.575 \pm 0.028$ & $-0.998 \pm 0.043$ & $2.443 \pm 0.025$ & 0.814 \\
\hline 481 & 684- 52523- 494 & $\mathrm{~J} 235242.19+002345.9$ & $1.180 \pm 0.081$ & $8.146 \pm 0.068$ & 8.037 & $-1.512 \pm 0.032$ & $-0.976 \pm 0.019$ & $2.315 \pm 0.029$ & 0.806 \\
\hline 482 & $684-52523-560$ & $\mathrm{~J} 235519.63+000051.8$ & $1.183 \pm 0.046$ & $8.187 \pm 0.038$ & 8.145 & $-1.424 \pm 0.015$ & $-0.945 \pm 0.011$ & $2.226 \pm 0.013$ & 0.747 \\
\hline 483 & $685-52203-296$ & $\mathrm{~J} 235519.63+000051.8$ & $0.993 \pm 0.057$ & $8.387 \pm 0.066$ & 8.087 & $-1.433 \pm 0.019$ & $-0.943 \pm 0.012$ & $2.236 \pm 0.017$ & 0.800 \\
\hline 484 & 686- 52519- 2 & J001042.84-010200.9 & $1.244 \pm 0.085$ & $8.161 \pm 0.066$ & 8.469 & $-1.416 \pm 0.028$ & $-1.090 \pm 0.024$ & $2.339 \pm 0.026$ & 0.855 \\
\hline 485 & 686- 52519- 185 & J000703.98-003447.6 & $1.258 \pm 0.032$ & $8.117 \pm 0.026$ & 8.191 & $-1.427 \pm 0.013$ & $-0.961 \pm 0.010$ & $2.272 \pm 0.011$ & 0.791 \\
\hline 486 & 686- 52519- 406 & J000657.02+005125.9 & $1.195 \pm 0.027$ & $8.177 \pm 0.025$ & 8.530 & $-1.336 \pm 0.008$ & $-0.979 \pm 0.008$ & $2.181 \pm 0.005$ & 0.797 \\
\hline 487 & 686- 52519- 476 & J000635.54+002333.3 & $1.317 \pm 0.087$ & $8.037 \pm 0.057$ & 8.097 & $-1.537 \pm 0.027$ & $-0.881 \pm 0.014$ & $2.341 \pm 0.024$ & 0.768 \\
\hline 488 & $691-52199-252$ & J004406.79-010838.4 & $1.280 \pm 0.039$ & $8.110 \pm 0.029$ & 8.147 & $-1.485 \pm 0.016$ & $-1.012 \pm 0.011$ & $2.353 \pm 0.014$ & 0.803 \\
\hline 489 & $691-52199-307$ & $\mathrm{~J} 004020.02+000008.6$ & $1.060 \pm 0.056$ & $8.221 \pm 0.053$ & 8.763 & $-0.942 \pm 0.007$ & $-0.816 \pm 0.015$ & $1.629 \pm 0.006$ & 0.750 \\
\hline 490 & $691-52199-573$ & J004859.14+005553.0 & $1.420 \pm 0.063$ & $7.928 \pm 0.035$ & 8.039 & $-1.541 \pm 0.029$ & $-0.913 \pm 0.015$ & $2.312 \pm 0.026$ & 0.753 \\
\hline 491 & $691-52199-636$ & J005147.30+000939.9 & $1.506 \pm 0.025$ & $7.833 \pm 0.020$ & 7.881 & $-1.858 \pm 0.021$ & $-1.256 \pm 0.011$ & $2.695 \pm 0.019$ & 0.878 \\
\hline 492 & 692- 52201- 394 & J005147.30+000939.9 & $1.617 \pm 0.027$ & $7.768 \pm 0.020$ & 7.888 & $-1.836 \pm 0.024$ & $-1.276 \pm 0.012$ & $2.683 \pm 0.022$ & 0.876 \\
\hline 493 & $693-52254-403$ & J010101.27+011040.0 & $1.144 \pm 0.066$ & $8.225 \pm 0.059$ & 8.225 & $-1.327 \pm 0.053$ & $-0.929 \pm 0.016$ & $2.171 \pm 0.050$ & 0.807 \\
\hline 494 & 693- 52254- 454 & J010112.24+005449.3 & $1.178 \pm 0.075$ & $8.173 \pm 0.058$ & 8.094 & $-1.460 \pm 0.020$ & $-0.822 \pm 0.016$ & $2.206 \pm 0.017$ & 0.701 \\
\hline 495 & 693- 52254- 463 & $\mathrm{~J} 010000.24+001727.2$ & $1.152 \pm 0.043$ & $8.259 \pm 0.042$ & 8.466 & $-1.480 \pm 0.020$ & $-1.150 \pm 0.027$ & $2.410 \pm 0.017$ & 0.867 \\
\hline 496 & 693- 52254- 480 & J005959.88+000014.0 & $1.483 \pm 0.092$ & $7.857 \pm 0.046$ & 8.041 & $-1.403 \pm 0.020$ & $-0.797 \pm 0.015$ & $2.108 \pm 0.017$ & 0.698 \\
\hline 497 & 694- 52209- 73 & J011210.87-000330.9 & $1.203 \pm 0.059$ & $8.154 \pm 0.042$ & 8.112 & $-1.391 \pm 0.014$ & $-0.837 \pm 0.011$ & $2.161 \pm 0.012$ & 0.720 \\
\hline 498 & 694- 52209- 231 & J010628.39-002000.9 & $1.368 \pm 0.048$ & $8.036 \pm 0.031$ & 8.144 & $-1.643 \pm 0.035$ & $-1.057 \pm 0.020$ & $2.531 \pm 0.034$ & 0.821 \\
\hline 499 & $695-52202-261$ & J011210.87-000330.9 & $1.200 \pm 0.057$ & $8.168 \pm 0.043$ & 8.139 & $-1.391 \pm 0.018$ & $-0.841 \pm 0.014$ & $2.164 \pm 0.016$ & 0.709 \\
\hline 500 & 698- 52203- 250 & J013525.58-011348.3 & $1.092 \pm 0.051$ & $8.262 \pm 0.047$ & 8.552 & $-1.209 \pm 0.029$ & $-0.893 \pm 0.013$ & $1.982 \pm 0.027$ & 0.751 \\
\hline 501 & 699- 52202- 337 & J014246.61+010450.5 & $1.368 \pm 0.052$ & $7.963 \pm 0.032$ & 8.119 & $-1.353 \pm 0.014$ & $-0.847 \pm 0.012$ & $2.117 \pm 0.011$ & 0.757 \\
\hline 502 & 701- 52179- 117 & J020223.52-002728.0 & $1.519 \pm 0.038$ & $7.820 \pm 0.026$ & 7.887 & $-1.812 \pm 0.025$ & $-1.133 \pm 0.015$ & $2.638 \pm 0.022$ & 0.870 \\
\hline 503 & 702- 52178- 335 & J020411.35+004304.8 & $1.173 \pm 0.050$ & $8.171 \pm 0.044$ & 8.429 & $-1.372 \pm 0.021$ & $-0.954 \pm 0.014$ & $2.194 \pm 0.018$ & 0.804 \\
\hline 504 & 703- 52209- 215 & J021514.66-005254.4 & $1.091 \pm 0.065$ & $8.298 \pm 0.060$ & 8.160 & $-1.408 \pm 0.018$ & $-0.885 \pm 0.015$ & $2.227 \pm 0.016$ & 0.762 \\
\hline 505 & 704- 52205- 42 & J022635.74-011021.3 & $1.173 \pm 0.068$ & $8.186 \pm 0.053$ & 8.114 & $-1.392 \pm 0.016$ & $-0.801 \pm 0.012$ & $2.163 \pm 0.014$ & 0.722 \\
\hline 506 & 704- 52205- 494 & J022322.78+002336.9 & $1.310 \pm 0.036$ & $8.037 \pm 0.026$ & 8.047 & $-1.525 \pm 0.016$ & $-0.938 \pm 0.009$ & $2.351 \pm 0.014$ & 0.804 \\
\hline 507 & $707-52177-11$ & J024939.72-011151.3 & $1.326 \pm 0.039$ & $8.035 \pm 0.031$ & 8.048 & $-1.626 \pm 0.014$ & $-1.087 \pm 0.010$ & $2.492 \pm 0.012$ & 0.842 \\
\hline 508 & 707- 52177- 369 & J024355.18+011148.8 & $1.093 \pm 0.070$ & $8.287 \pm 0.066$ & 8.562 & $-1.226 \pm 0.016$ & $-0.984 \pm 0.022$ & $2.059 \pm 0.013$ & 0.791 \\
\hline 509 & 707- 52177- 525 & $\mathrm{~J} 024702.71+011529.5$ & $1.285 \pm 0.022$ & $8.099 \pm 0.021$ & 8.650 & $-1.430 \pm 0.010$ & $-1.132 \pm 0.011$ & $2.337 \pm 0.008$ & 0.863 \\
\hline 510 & 708- 52175- 147 & J025540.01-002240.0 & $1.187 \pm 0.081$ & $8.189 \pm 0.061$ & 8.163 & $-1.434 \pm 0.035$ & $-0.871 \pm 0.017$ & $2.216 \pm 0.034$ & 0.713 \\
\hline 511 & 708- 52175- 155 & J025436.22+000339.9 & $1.443 \pm 0.040$ & $7.890 \pm 0.024$ & 7.983 & $-1.691 \pm 0.016$ & $-1.059 \pm 0.009$ & $2.483 \pm 0.015$ & 0.805 \\
\hline 512 & 708- 52175- 172 & J025324.24-000716.6 & $1.276 \pm 0.063$ & $8.100 \pm 0.044$ & 8.211 & $-1.424 \pm 0.033$ & $-0.901 \pm 0.013$ & $2.245 \pm 0.031$ & 0.763 \\
\hline 513 & 708-52175- 299 & J025020.86-005828.9 & $1.338 \pm 0.087$ & $7.962 \pm 0.048$ & 8.493 & $-1.052 \pm 0.009$ & $-0.664 \pm 0.009$ & $1.658 \pm 0.007$ & 0.599 \\
\hline 514 & 708- 52175- 579 & J025536.26+005609.6 & $1.235 \pm 0.071$ & $8.069 \pm 0.047$ & 8.106 & $-1.249 \pm 0.010$ & $-0.729 \pm 0.008$ & $1.924 \pm 0.008$ & 0.671 \\
\hline 515 & $710-52203-611$ & $\mathrm{~J} 031314.21+004726.5$ & $1.506 \pm 0.126$ & $7.903 \pm 0.059$ & 8.163 & $-1.270 \pm 0.063$ & $-0.751 \pm 0.022$ & $1.962 \pm 0.059$ & 0.621 \\
\hline 516 & 712-52179- 108 & J032708.26-002551.9 & $1.212 \pm 0.071$ & $8.212 \pm 0.055$ & 8.186 & $-1.421 \pm 0.020$ & $-0.878 \pm 0.052$ & $2.292 \pm 0.017$ & 0.762 \\
\hline 517 & 712- 52199- 115 & J032708.26-002551.9 & $1.209 \pm 0.057$ & $8.205 \pm 0.046$ & 8.235 & $-1.392 \pm 0.018$ & $-0.833 \pm 0.051$ & $2.267 \pm 0.016$ & 0.779 \\
\hline 518 & 713- 52178- 213 & J033211.81-004416.0 & $1.785 \pm 0.195$ & $7.588 \pm 0.064$ & 8.686 & $-0.844 \pm 0.008$ & $-0.645 \pm 0.011$ & $1.275 \pm 0.004$ & 0.480 \\
\hline 519 & 713- 52178- 496 & J033319.20+001731.2 & $1.248 \pm 0.036$ & $8.136 \pm 0.028$ & 8.403 & $-1.264 \pm 0.026$ & $-0.942 \pm 0.011$ & $2.118 \pm 0.024$ & 0.790 \\
\hline 520 & 714- 52201- 91 & J034254.29-005520.2 & $1.046 \pm 0.060$ & $8.332 \pm 0.060$ & 8.169 & $-1.378 \pm 0.031$ & $-0.903 \pm 0.014$ & $2.179 \pm 0.030$ & 0.770 \\
\hline 521 & $802-52289-423$ & $\mathrm{~J} 030135.57+011420.0$ & $1.340 \pm 0.037$ & $8.059 \pm 0.034$ & 7.907 & $-1.884 \pm 0.054$ & $-1.481 \pm 0.031$ & $2.857 \pm 0.051$ & 0.940 \\
\hline 522 & $804-52286-85$ & J031918.24-005859.1 & $1.093 \pm 0.067$ & $8.298 \pm 0.062$ & 8.186 & $-1.446 \pm 0.020$ & $-0.969 \pm 0.012$ & $2.276 \pm 0.018$ & 0.773 \\
\hline 523 & 805- 52586- 144 & J032708.26-002551.9 & $1.243 \pm 0.038$ & $8.174 \pm 0.030$ & 8.147 & $-1.364 \pm 0.013$ & $-0.839 \pm 0.049$ & $2.257 \pm 0.011$ & 0.797 \\
\hline 524 & $805-52586-482$ & J032713.08+003112.3 & $1.108 \pm 0.041$ & $8.249 \pm 0.037$ & 8.201 & $-1.314 \pm 0.012$ & $-0.856 \pm 0.007$ & $2.115 \pm 0.010$ & 0.776 \\
\hline 525 & $805-52586-550$ & $\mathrm{~J} 032750.16+010135.0$ & $1.006 \pm 0.045$ & $8.355 \pm 0.041$ & 8.471 & $-1.106 \pm 0.006$ & $-0.695 \pm 0.005$ & $1.747 \pm 0.004$ & 0.614 \\
\hline 526 & $805-52586-605$ & J033128.46+003737.2 & $1.289 \pm 0.087$ & $8.036 \pm 0.058$ & 8.056 & $-1.473 \pm 0.020$ & $-0.832 \pm 0.011$ & $2.181 \pm 0.016$ & 0.682 \\
\hline 527 & 806- 52592- 587 & J033653.64+005657.4 & $1.285 \pm 0.077$ & $8.055 \pm 0.047$ & 8.100 & $-1.456 \pm 0.022$ & $-0.823 \pm 0.018$ & $2.151 \pm 0.019$ & 0.649 \\
\hline 528 & $811-52669-415$ & J030903.89+003846.6 & $1.512 \pm 0.049$ & $7.932 \pm 0.034$ & 8.071 & $-1.844 \pm 0.037$ & $-1.267 \pm 0.022$ & $2.801 \pm 0.034$ & 0.903 \\
\hline 529 & $960-52466-61$ & $\mathrm{~J} 134225.32+582432.7$ & $1.158 \pm 0.034$ & $8.194 \pm 0.032$ & 8.486 & $-1.393 \pm 0.010$ & $-0.999 \pm 0.011$ & $2.232 \pm 0.008$ & 0.823 \\
\hline 530 & $972-52428-368$ & $\mathrm{~J} 165844.50+351923.1$ & $1.125 \pm 0.036$ & $8.216 \pm 0.031$ & 8.317 & $-1.215 \pm 0.008$ & $-0.792 \pm 0.007$ & $1.957 \pm 0.006$ & 0.715 \\
\hline 531 & $978-52431-415$ & $\mathrm{~J} 171400.93+313023.4$ & $1.024 \pm 0.048$ & $8.335 \pm 0.046$ & 8.608 & $-1.035 \pm 0.008$ & $-0.842 \pm 0.014$ & $1.749 \pm 0.006$ & 0.696 \\
\hline
\end{tabular}

